Florida International University FIU Digital Commons

FIU Electronic Theses and Dissertations

University Graduate School

$1-2018$

\title{
Anomalous Properties of Sub-10-nm Magnetic Tunneling Junctions
}

Mark Stone

Florida International University, mston002@fiu.edu

DOI: 10.25148 /etd.FIDC006593

Follow this and additional works at: https:// digitalcommons.fiu.edu/etd

Part of the Electromagnetics and Photonics Commons, and the Nanotechnology Fabrication Commons

\section{Recommended Citation}

Stone, Mark, "Anomalous Properties of Sub-10-nm Magnetic Tunneling Junctions" (2018). FIU Electronic Theses and Dissertations. 3640.

https://digitalcommons.fiu.edu/etd/3640

This work is brought to you for free and open access by the University Graduate School at FIU Digital Commons. It has been accepted for inclusion in FIU Electronic Theses and Dissertations by an authorized administrator of FIU Digital Commons. For more information, please contact dcc@fiu.edu. 


\section{FLORIDA INTERNATIONAL UNIVERSITY}

Miami, Florida

\section{ANOMALOUS PROPERTIES OF SUB-10-NM MAGNETIC TUNNELING JUNCTIONS}

A dissertation submitted in partial fulfillment of

the requirements for the degree of

DOCTOR OF PHILOSOPHY

in

ELECTRICAL ENGINEERING

by

Mark A. Stone

2018 
To: Dean John L. Volakis

College of Engineering and Computing

This dissertation, written by Mark A. Stone, and entitled Anomalous Properties of Sub10-nm Magnetic Tunneling Junctions, having been approved in respect to style and intellectual content, is referred to you for judgment.

We have read this dissertation and recommend that it be approved.

Jean Andrian

Irene Calizo

Pezhman Mardanpour

Sakhrat Khizroev, Major Professor

Date of Defense: January 30, 2018

The dissertation of Mark A. Stone is approved.

Dean John L. Volakis

College of Engineering and Computing

Andrés G. Gil

Vice President for Research and Economic Development and Dean of the University Graduate School

Florida International University, 2018 
(C) Copyright 2018 by Mark A. Stone

All rights reserved. 


\section{DEDICATION}

I dedicate my dissertation to my wife, family, friends, and colleagues for being supportive of me throughout my academic and personal life. Without them none of this would have been possible. I hope to be able to do the same for others when the moment presents itself. 


\section{ACKNOWLEDGMENTS}

I would like to express my appreciation to my advisor Dr. Sakhrat Khizroev for all his insight and support which has made the completion of this study possible. Secondly, I want to acknowledge the NSF Center Energy Efficient Electronics (E3S) for both the financial support throughout this research and the platform of shared knowledge they have created. I would like to acknowledge my committee members Dr. Irene Calizo, Dr. Jean Andrian and Dr. Pezhman Mardanpour for their support along the way. I would like to acknowledge my classmates/friends for all the motivational talks and fun times Emmanuel Stimphil, Krystine Pimentel, Kevin Luongo, Brayan Navarrete, Ping Wang, Jeongmin Hong, Ali Hadjikhani, and Rakesh Guduru. Finally, I would like to acknowledge and thank my beautiful amazing wife Jessenia for her patience, love, and support. My family and my wife's family for all their love and support. Finally, my mom for teaching me how to persevere and to keep going even when the tank is empty. 


\section{ABSTRACT OF THE DISSERTATION}

ANOMALOUS PROPERTIES OF SUB-10-NM MAGNETIC TUNNELING

JUNCTIONS

by

Mark A. Stone

Florida International University, 2018

Miami, Florida

\section{Professor Sakhrat Khizroev, Major Professor}

Magnetic Logic Devices have the advantage of non-volatility, radiation hardness, scalability down to the sub-10nm range, and three-dimensional (3D) integration capability. Despite these advantages, magnetic applications for information processing remain limited. The main stumbling block is the high energy required to switch information states in spin-based devices. Recently, the spin transfer torque (STT) effect has been introduced as a promising solution. STT based magnetic tunneling junctions (MTJs), use a spin polarized electric current to switch magnetic states. They are theorized to bring the switching energy down substantially. However, the switching current density remains in the order of $1 \mathrm{MA} / \mathrm{cm} 2$ in current STT-MTJ devices, with the smallest device reported to date around $10 \mathrm{~nm}$. This current density remains inadequately high for enabling a wide range of information processing applications. For this technology to be competitive in the near future it is critical to show that it could be favorably scaled into the sub-10-nm range. This is an intriguing size range that currently remains unexplored. Nanomagnetic devices may display promising characteristics that can make them superior to their semiconductor counterparts. Below $10 \mathrm{~nm}$ the spin physics from the 
surface become dominate versus those due to volume. The goal is to understand the size dependence versus the switching current. 


\section{TABLE OF CONTENTS}

CHAPTER

PAGE

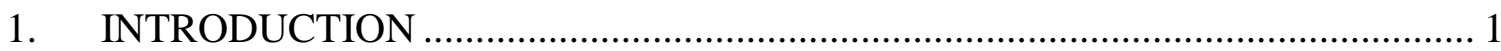

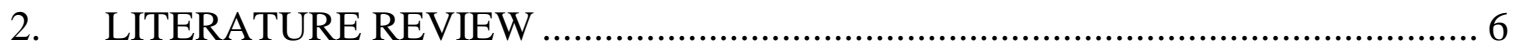

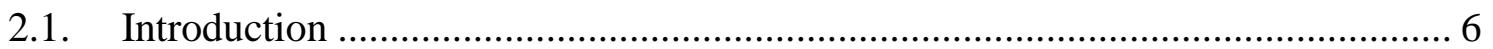

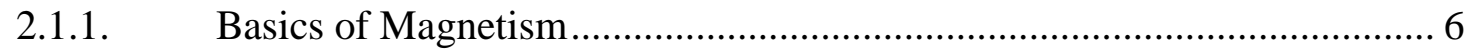

2.1.2. Magnetism of Electrons ……………............................................... 14

2.1.3. Classification of Magnetic Materials ……............................................... 22

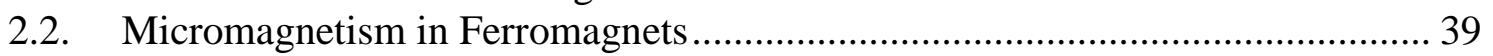

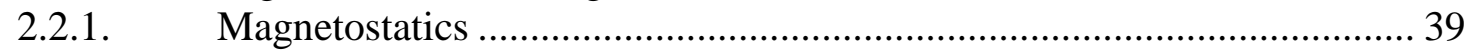

2.2.2. Magnetic Anisotropy ………………................................................ 40

2.2.3. Magnetostriction …………............................................................. 42

2.2.4. Zeeman Energy ………................................................................... 42

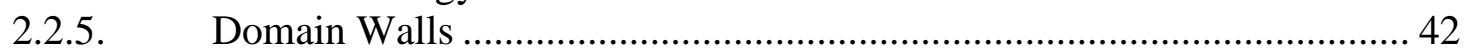

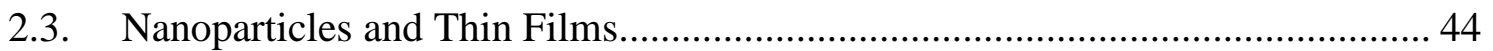

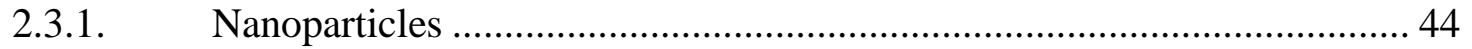

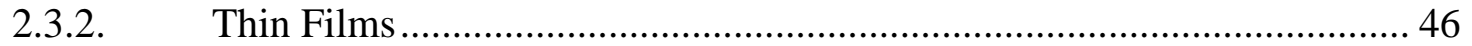

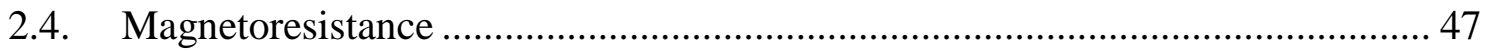

2.4.1. Anisotropicmagnetoresistance ........................................................... 48

2.4.2. Giantmagnetoresistance ……............................................................ 49

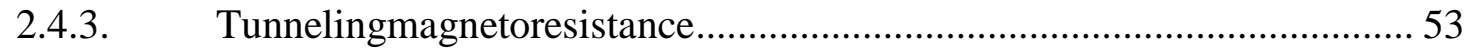

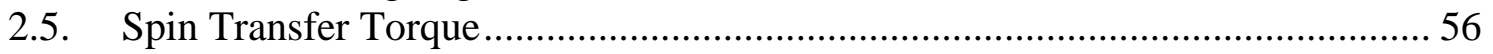

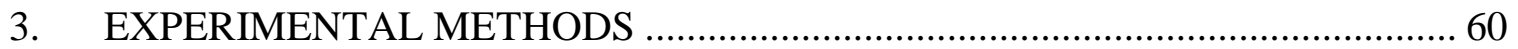

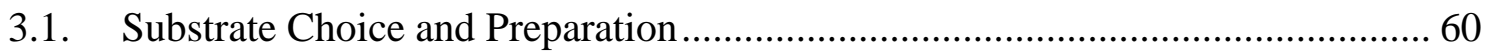

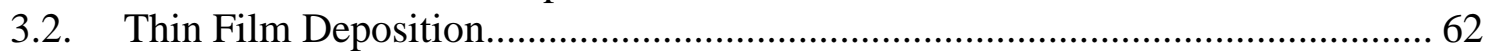

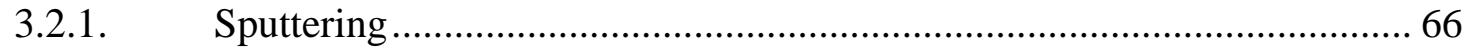

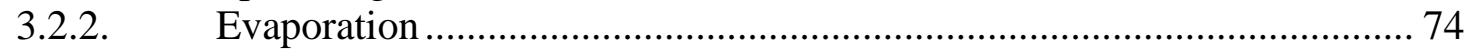

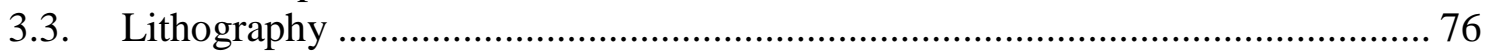

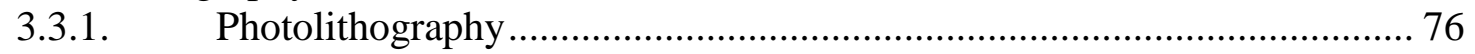

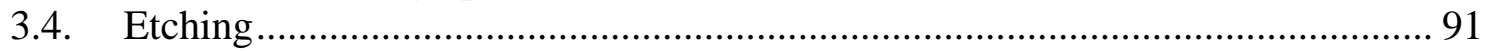

3.4.1. Focused Ion Beam................................................................................ 94

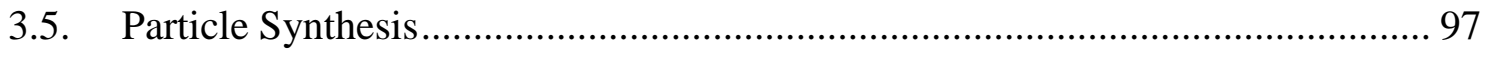

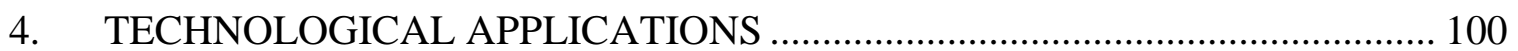

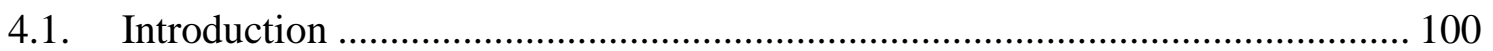

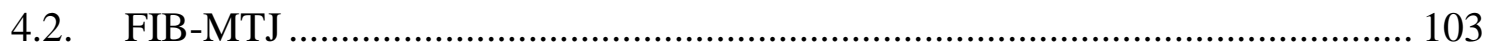

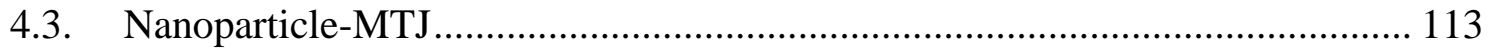

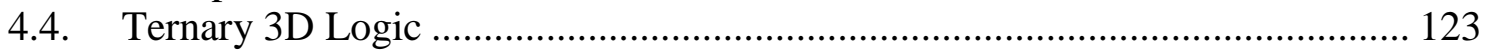

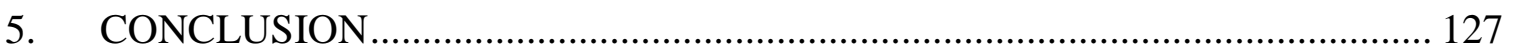

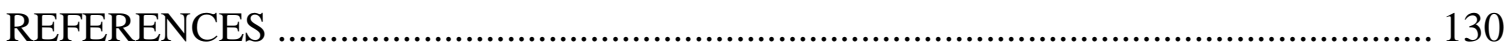




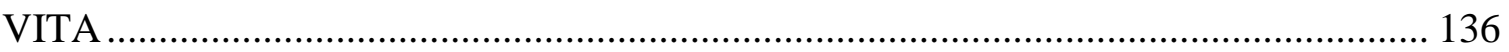




\section{LIST OF TABLES}

TABLE

PAGE

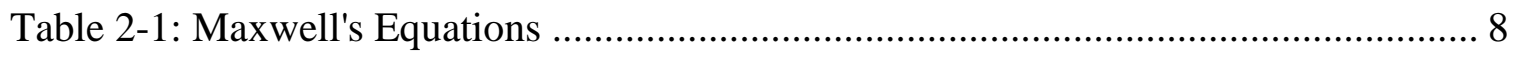

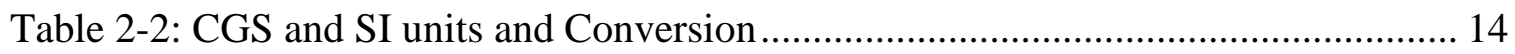

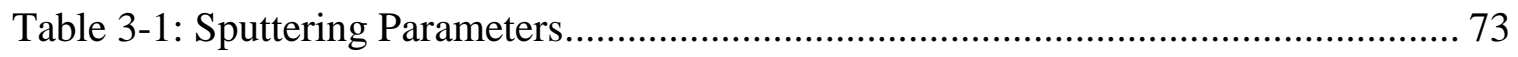

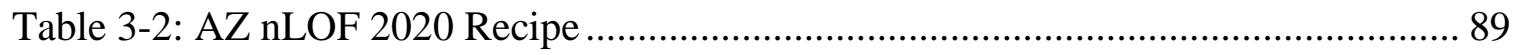

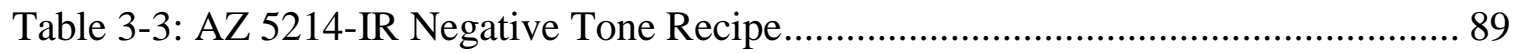

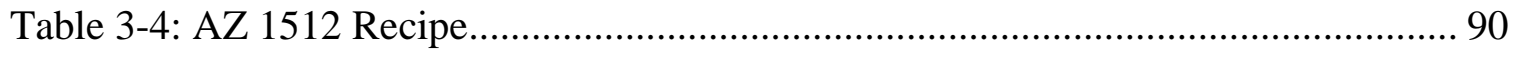

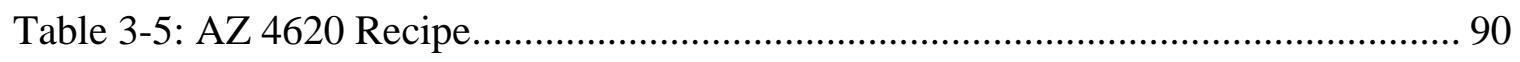

Table 3-6: AZ 5214 Positive Tone Recipe ................................................................... 91 


\section{LIST OF FIGURES}

FIGURE

PAGE

Figure 1-1: Memory Capacity VS Latency of Competing Technologies ......................... 4

Figure 2-1: Magnetic Field Lines .............................................................................. 7

Figure 2-2: Relation between Magnetic Fields and Current ........................................ 8

Figure 2-3: Flux Density In and Out of a Material ................................................... 11

Figure 2-4: Magnetization Curves for Different Materials ........................................ 12

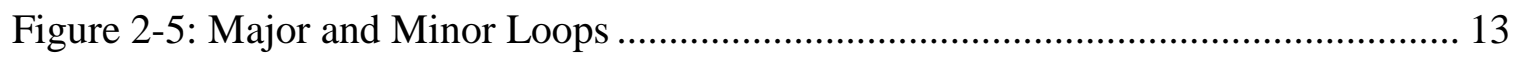

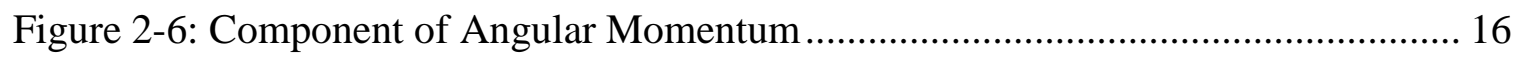

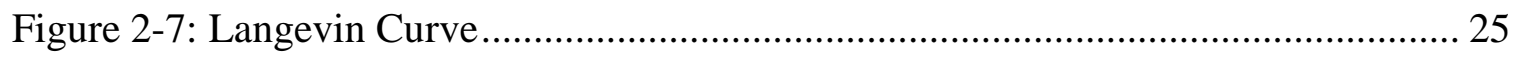

Figure 2-8: Electron Energy Adjustment with an Applied Field .................................. 29

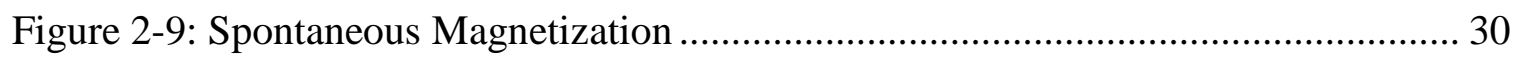

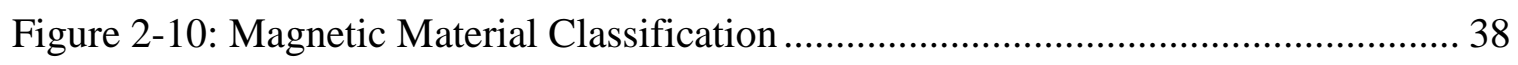

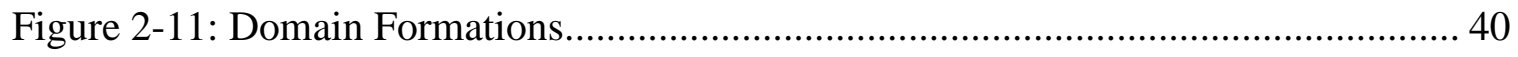

Figure 2-12: AMR Low and High Resistance ...................................................... 48

Figure 2-13: High and Low Resistance in GMR Junctions ....................................... 51

Figure 2-14: Parallel VS Antiparallel Configuration................................................ 52

Figure 2-15: Spins tunneling through barrier (a); MTJ Structure (b) ............................. 54

Figure 2-16: Different levels of polarization in a ferromagnet...................................... 55

Figure 2-17: Spin transfer torque in both directions ................................................ 57

Figure 2-18: Spin Transfer Torque Illustration..................................................... 58

Figure 3-1: Orange Peel Coupling Due to Surface Roughness.................................... 66

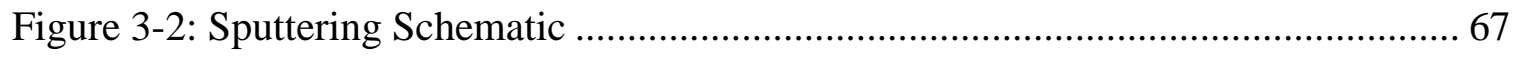

Figure 3-3: Magnetron Sputtering Process ......................................................... 70 


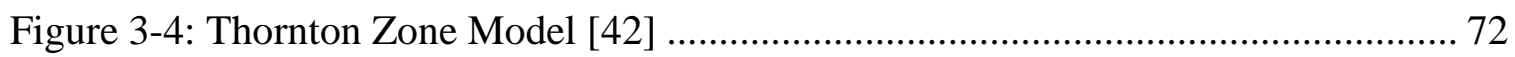

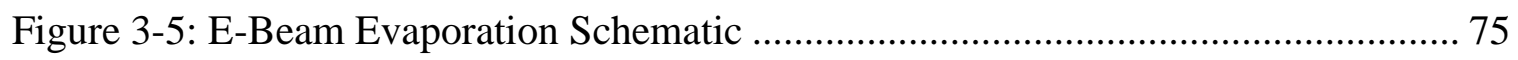

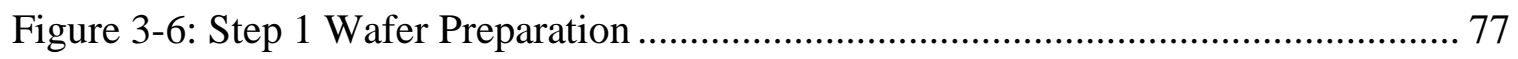

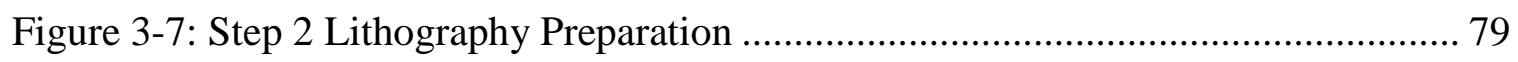

Figure 3-8: Light Field and Dark Field Masks ................................................... 79

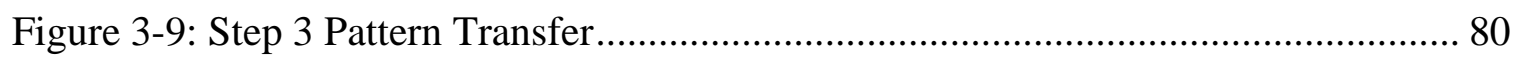

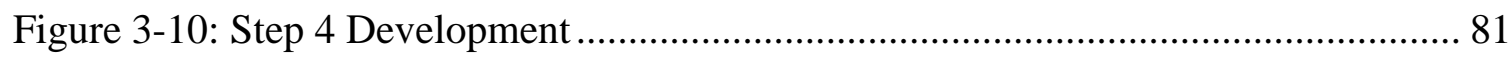

Figure 3-11: Step 5 Material Processing .................................................................. 82

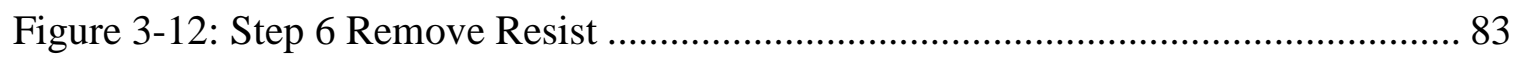

Figure 3-13: Negative and Positive Resist Exposure................................................ 84

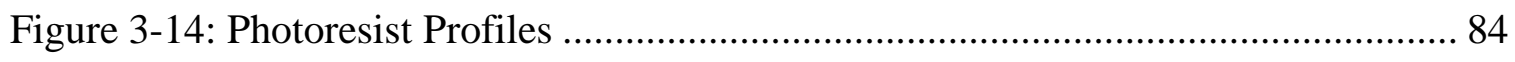

Figure 3-15: Lift-Off with Overcut (top) and Undercut (bottom) Sidewall Profiles ........ 85

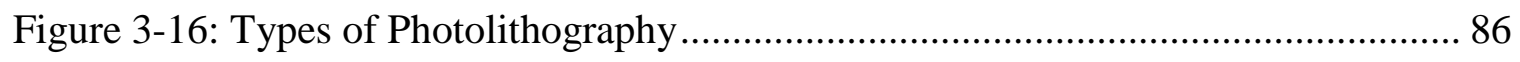

Figure 3-17: Light Intensity Profile on Mask with Grating [42] .................................. 88

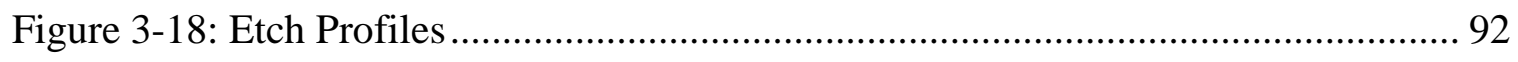

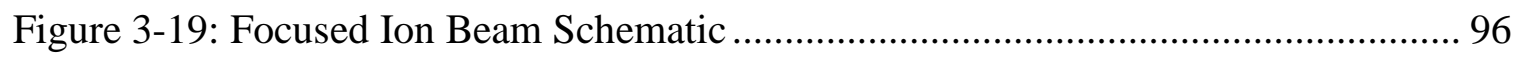

Figure 4-1:Ab Initio Quantum Mechanical Calculations [66] .................................... 105

Figure 4-2: Nanoprobe Device Schematic (left) and SEM image of the probe

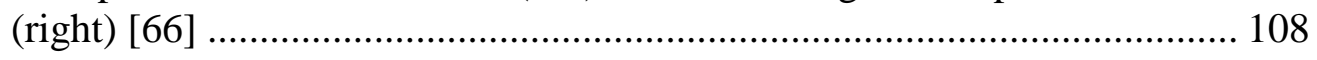

Figure 4-3: TMR Graph (left) and Switching Current vs Field Dependence (right)

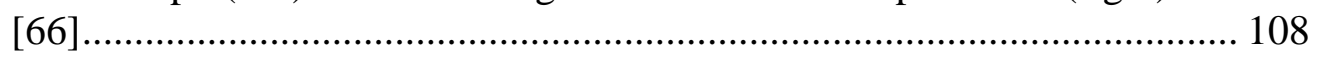

Figure 4-4: Microfabrication Process for developing MTJs ..................................... 110

Figure 4-5: Nanofabrication Process for developing MTJs ...................................... 111

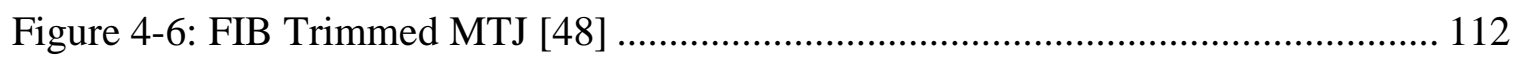

Figure 4-7: IV Measurement (left) and MR Measurement (right) [48] ........................ 112 
Figure 4-8: Particle Based MTJ Structure (left) and Schematic of Energy Levels with Fermi Energy Controlled by a Magnetic Field (right)

Figure 4-9: Thin Film Schematic of Particle Based Magnetic Tunnel Junction (left) and Cross-Sectional View of Particle Based Magnetic Tunnel Junction (right)

Figure 4-10: MFM Image (left) and High-resolution TEM Image of 2-nm

$\mathrm{CoFe} 2 \mathrm{O} 4$ Nanoparticles Sandwiched in a Junction (right) 116

Figure 4-11: TEM Images of Nanoparticles MTJ (left) and Magnification to see nanoparticles (right).....

Figure 4-12: Gallium Fibbed Magnetic Tunnel Junction (left) and TEM Images of the Cross Section of the MTJ (middle \& right)

Figure 4-13: MR for 4nm particles (left) and 10nm particles (right) 118

Figure 4-14: MR for 2nm Particles (Left and right graphs come from different batches)

Figure 4-15: IV measurement of 2nm-Nanoparticle MTJ with evidence of single electron transport

Figure 4-16: Nanoparticle magnetization with respect to the different points on the IV curve from Figure 4-15.

Figure 4-17: IV measurement for $2 \mathrm{~nm}$ particles

Figure 4-18: MR done at 50nA (top) and MR done at 10nA (bottom)

Figure 4-19: MH-Loop of MTJ Structure (left) and MTJ Composition (right) [69] 124

Figure 4-20: I-V curve measured via sweeping voltage in one direction (left) and the reverse direction (right) [69] 125

Figure 5-1: Switching Currents and TMR from Fabricated Devices 129 


\section{LIST OF ACRONYMS}

\begin{tabular}{|c|c|}
\hline AFM & Antiferromagnet \\
\hline AGM & Alternating Gradient Magnetometer \\
\hline AMR & Anisotropicmagnetoresistance \\
\hline $\mathrm{AC}$ & Alternating current \\
\hline CVD & Chemical Vapor Deposition \\
\hline $\mathrm{DC}$ & Direct current \\
\hline DRAM & Dynamic Random Access Memory \\
\hline $\mathrm{FM}$ & Ferromagnet \\
\hline GMR & Giantmagnetoresistance \\
\hline HMDS & Mexamethyldisilazane \\
\hline MFM & Magnetic Force Microscopy \\
\hline MR & Magnetoresistance \\
\hline MTJ & Magnetic Tunnel Junction \\
\hline MOKE & Magneto-optical Kerr Effect \\
\hline PMMA & Polymethylmethacrylate \\
\hline PVD & Physical Vapor Deposition \\
\hline $\mathrm{RF}$ & Radio Frequency \\
\hline SRAM & Static Random Access Memory \\
\hline STT & Spin Transfer Torque \\
\hline TMR & Tunnelingmagnetoresistance \\
\hline UHV & Ultra High Vacuum \\
\hline UV & Ultraviolet \\
\hline
\end{tabular}




\section{INTRODUCTION}

Gordon E. Moore predicted the doubling of transistors in an integrated circuit roughly every 2 years. His prediction proved accurate for several decades and it eventually became known as Moore's Law in the semiconductor industry. A more accurate analysis

describes the chip performance doubling every 18 months given the combined effect of adding more transistors in a chip and increasing their performance [1]. This trend, however, cannot go on indefinitely. Moore's Law is reaching saturation and prolonging its growth has been a major focus of research in universities and industry. This thesis will address this issue by developing devices from a magnetics stand point and are theorized to push Moore's law further.

Moore's law is hitting a brick wall and further scaling of the CMOS transistor is reaching its limit both technologically and economically. When scaling a CMOS transistor, the channel length is reduced and the gate dielectric is decreased. We begin to encounter physical limitations such as when the channels become closer, a higher off-state drain leakage current will flow. Also, as the thickness of the dielectric decreases, quantum mechanical tunneling begins to take place and we have gate leakage currents that increase exponentially as the dielectric thins down. Furthermore, the supply voltage has been unable to scale with equal footing with the transistor, causing the power density to grow [2]. In fact, as CMOS transistors scale down, static power density (device switched off) approaches the dynamic power density (device switched on). This contributes to further power and thermal problems that is adverse to the performance of the CMOS transistor. Improvement in the on/off ratio is an important issue to address in new devices [1-2].

We are living in the age of information technology where the Internet of things is 
dominating our lives. There has been rapid advancement in cloud computing, social networking, mobile Internet, etc. This progress comes at an enormous power cost in information processing. Growth in information technology has the world currently consuming $\sim 1.4 \%$ of the total electricity production in the form of data centers and servers, computers, etc [3]. It is important that research also finds a way to bring down the energy costs in the information processing industry. Landauer calculated that the minimum energy to switch a bit of information is $k T \ln 2$ [4], which is over a factor of five less than what modern transistors use. This thesis will focus on exploring new methods and concepts to reduce the energy consumption required to manipulate bits of information.

Spintronics is a field that studies the intrinsic spin of an electron and its associated magnetic moment. The purpose of this thesis is to develop devices that exploit spin properties of electrons in addition to its charge. What propelled the field of spintronics was the discovery of Giantmagnetoresistance (GMR), which became the backbone of the magnetic storage industry [2]. GMR is an effect found in alternating thin metallic films of magnetic and non-magnetic layers. There is a substantial difference in electrical resistance that depends on the magnetization of the magnetic films [5]. It is a quantum mechanical effect whose discovery warranted the 2007 Nobel Prize in physics, awarded to Albert Fert and Peter Grünberg. Tunnelingmagnetoresistance (TMR) is a concept built off GMR and led to the development of the modern magnetic tunnel junction (MTJ) that are ubiquitous in read-heads in hard disk drives (HDD) and niche market non-volatile memory technologies such as MRAM. 
Traditionally, external magnetic fields have been used to flip magnetic elements. This requires relatively high power, slow speed actuation (mechanically moving parts), and architectural complexity [6]. Furthermore, as devices are scaled down, accidental "writing" can occur as fields switch neighboring devices unintentionally [7]. Spintransfer torque (STT) has been proposed as a switching solution that has theoretical lower power consumption and better scalability than applied external fields. It consists of switching the magnetic layer with a spin-polarized current. While STT is promising, currents required to switch states remain inadequately high for large information processing applications [8-9].

The few studies that have explored the sub $10 \mathrm{~nm}$ region in magnetic media, suggest that the switching current reduction in this region is superior to what would be expected by linear scaling [10]. This size range offers a solution in the form of energy efficient STTMTJ devices. Spin transfer torque (STT) based Magnetic Tunneling Junctions (MTJ) is a promising technology that can overcome many challenges from CMOS devices. STTMTJs have the potential to be a universal memory that has the speed advantage of Static Random Access Memory (SRAM), the packing density of Dynamic Random Access Memory (DRAM), and the non-volatility of FLASH memory [11-13]. Because of the non-volatility of magnets these devices can perform logic operations with near-zero static power consumption. Their non-volatility has already made them popular in the data storage industry with the development of hard disk drives (HDD). However, to utilize them for logic operations the key challenge is addressing the high energy required to switch magnetic orientations. The smallest switching current to date is in the order of $1 \mathrm{MA} / \mathrm{cm}^{2}$, which is too high for enabling a wide range of information processing 
applications. Other technologies such as phase changing memory (PCM) and resistive random access memory (ReRAM) are among the competitors to create a universal memory. For STT memory technology to outclass CMOS, it is paramount to bring the switching current down substantially.

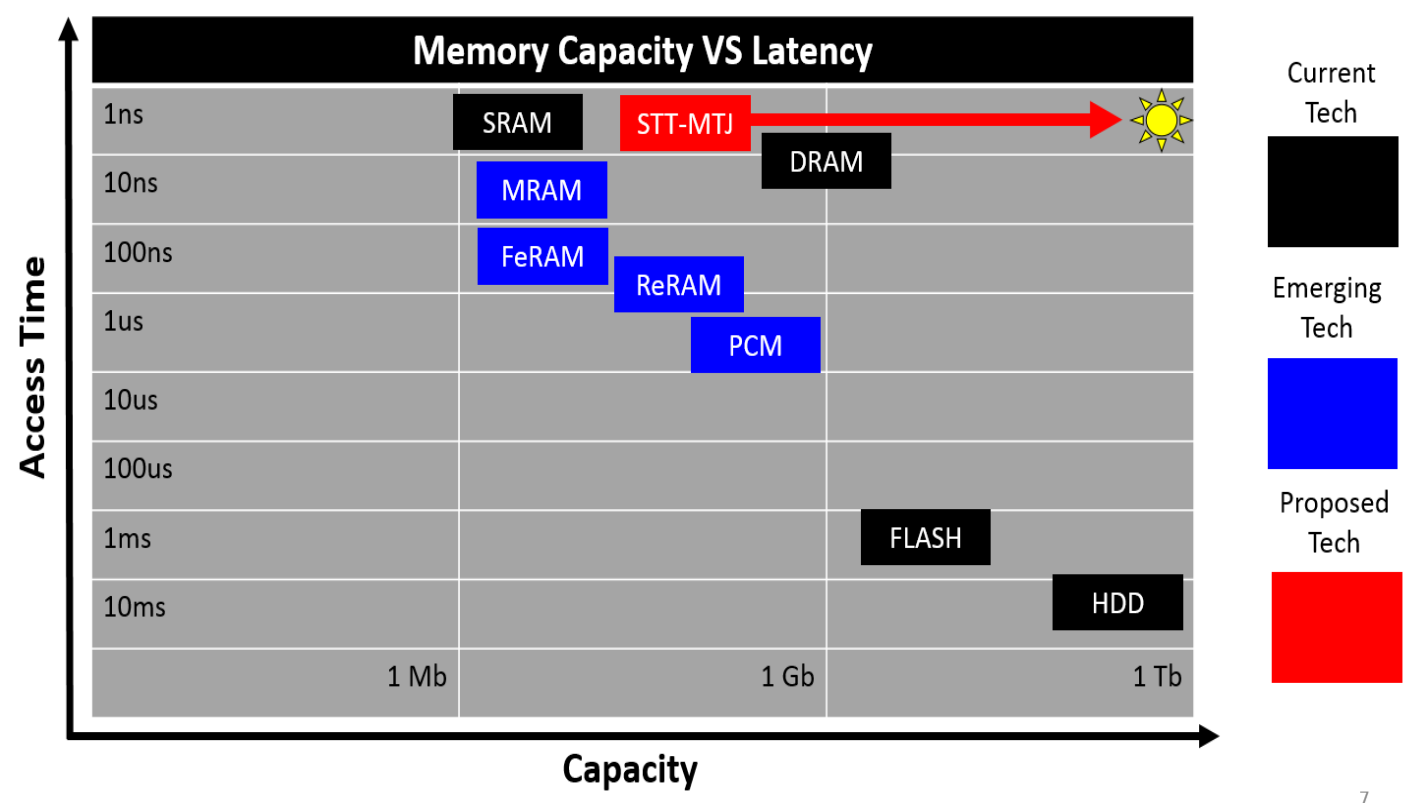

Figure 1-1: Memory Capacity VS Latency of Competing Technologies

This thesis focuses on the study and fabrication of STT-MTJs in the sub-10nm range. To date, the smallest reported device has a cross-section in the order of $\sim 10 \mathrm{~nm}$. So far, the switching current has scaled linearly with size, but in the sub-10nm range we can expect a dramatic reduction beyond linear scaling [10]. In fact, little is known in this size range and we theorize that these devices display promising characteristics that can overcome many of the challenges from their semiconductor counterparts. The biggest challenge in this thesis is fabricating nano-scale devices. Different micromachining methods (photolithography, etching, etc.) and nanofabrication techniques (Focused Ion beam) will be used in clever ways to meet that challenge. Most processes involved in making devices in the sub-10nm range are top-down. Particle based MTJs, on the other hand, are 
proposed as bottoms up method to address the size challenge in a novel way. Cobalt ferrite $\left(\mathrm{CoFe}_{2} \mathrm{O}_{4}\right)$ nanoparticles of different sizes are embedded into the MTJ architecture. These nanoparticles have unique size dependent magnetic properties that will also be explored in this thesis.

This thesis is broken down into five chapters. The first chapter (current) is an introduction to the thesis where I discuss the motivation of my research. The second chapter focuses on magnetism and the theoretical considerations. Chapter three goes over the fabrication techniques and challenges involved in making the devices in this thesis. Chapter four goes into further detail in the physics of both the STT-MTJs and the particle based MTJs. The process flow in making both the film and particle based MTJ are discussed, and the data obtained from the devices will be presented also. Chapter five is the conclusion where the impact of the results obtained is discussed and potential future work is explored. 


\section{LITERATURE REVIEW}

The role of magnetism has a major impact in the behavior of the devices fabricated in this thesis. This section will review basic concepts and definitions. Certain aspects of magnetism that pertains to the thesis will be covered in more detail. Some concepts unrelated to magnetics will also be discussed as needed. Having a proper understanding of the theory allows us to properly model and design a process flow for making devices. The majority of the information written in this section can be referenced from three excellent magnetics books: Magnetic Materials [14] by Nicola A. Spaldin, Introduction to Magnetic Materials [15] by B.D. Cullity, and Magnetism and Magnetic Materials [16] by J. M. D. Coey.

\subsection{Introduction}

\subsubsection{Basics of Magnetism}

Historically, a magnetic field $H$ was thought of as the field surrounding a magnetic pole that exerts a force on another pole nearby, much like Coulomb's law for interacting charged particles. The north pole acts as a source of magnetic field where lines of forces radiate outwards, and the south as a sink where they converge. The field strength can be understood quantitatively as the number of lines of force passing through a unit area perpendicular to the field. For example, in Gaussian units (CGS) a unit for magnetic field strength is Oersteds where 1Oe is 1 "line of force" passing through $1 \mathrm{~cm}^{2}$. The number of lines of force is expressed as the amount of flux $\phi$, where the unit of flux in CGS is the Maxwell (Mx) and each line of force is $1 \mathrm{Mx}$ [15]. 


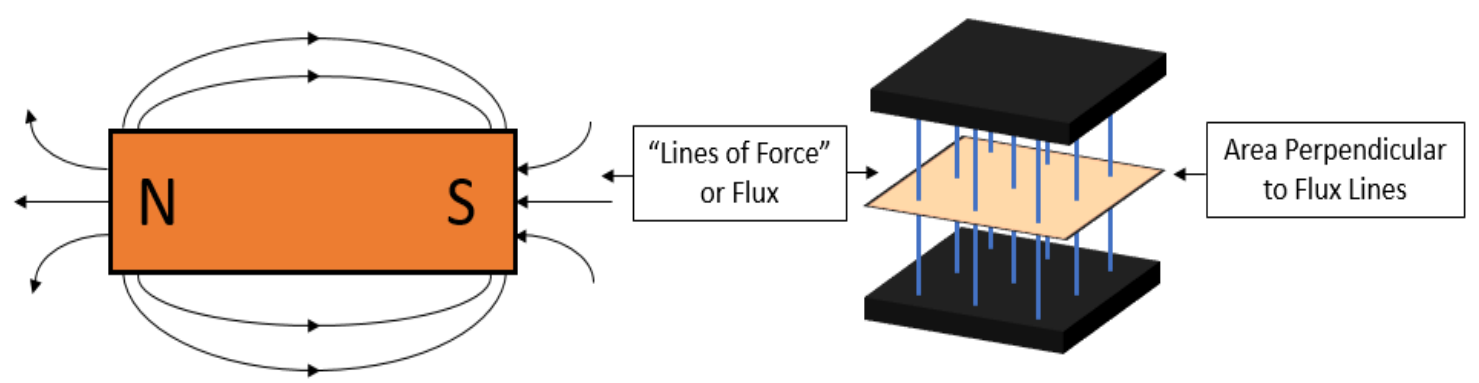

Figure 2-1: Magnetic Field Lines

The concept of flux is important because Faraday's law of electromagnetic induction tells us that a change in flux generates an electromotive force $\varepsilon$. This electromotive force creates a potential difference that leads to an electric current in a closed circuit. Mathematically, Faraday's law is expressed as: $\varepsilon=-\frac{d \phi}{d t}$. The greater the change in flux, the greater the generated voltage. The negative sign is attributed to Lenz's law where the current in the circuit creates a field in the opposite direction of the flux. Consequently, Ampère discovered that currents also generate magnetic fields. Ampère experimentally found that a magnetic field, equivalent to that of a bar magnet, was found in circular current carrying conductors. He concluded that all magnetic effects originate from current loops and those materials that exhibit magnet effects are due to "molecular currents." His experiments led to Ampère's law that relates the magnetic field to the amount of current in a conductor: $\oint H \cdot d l=I$. Maxwell would amend Ampère's law by adding a displacement current $I_{D}=\varepsilon_{0} \frac{\partial \mathrm{E}}{\partial \mathrm{t}}$. Maxwell reasoned that if a time varying magnetic field generates an electric field (Faraday's law), the reverse must also be true. A more general (but equivalent) form of Ampère's law that is often used is the Biot-Savart law that states $\delta \overrightarrow{\boldsymbol{H}}=\frac{1}{4 \pi R^{2}} I \delta \overrightarrow{\boldsymbol{l}} \times \overrightarrow{\boldsymbol{R}}$, where $\delta \boldsymbol{H}$ is the field created by the current $I$ at $\delta \boldsymbol{l}$ of the conductor 
at a distance $\boldsymbol{R}$. Ampere's law is preferred over the Biot-Savart law for calculating magnetic fields in configurations with a high degree of symmetry.

Faraday's Law

Magnet Movement creates change of flux

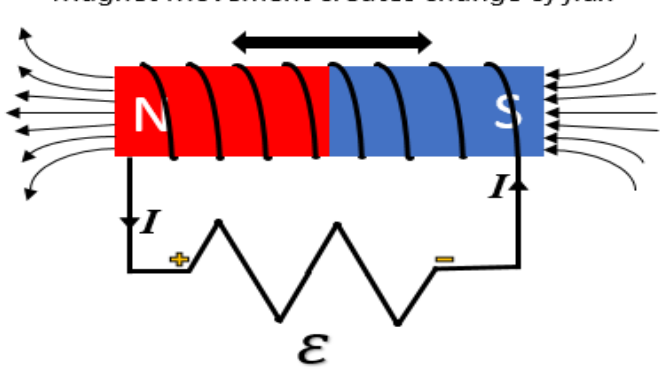

Ampere's Law

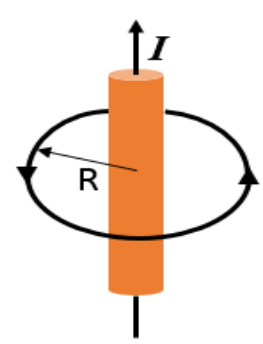

Current Loop

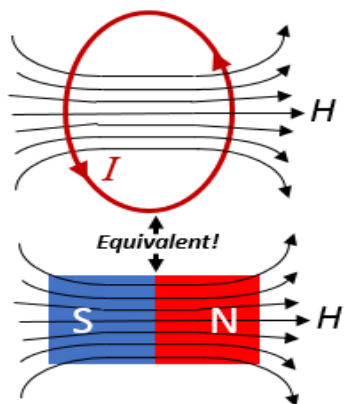

Figure 2-2: Relation between Magnetic Fields and Current

The discoveries made by Faraday and Ampere (among others) help unify the two phenomena of electric currents and magnetism into the field of electromagnetism. The relation between electric and magnetic forces form the foundation of classical electromagnetism and are summarized by Maxwell in his famous four equations known as Maxwell's Equations. They can be expressed in both differential and integral form:

Table 2-1: Maxwell's Equations

\begin{tabular}{|l|c|c|}
\hline \multicolumn{2}{|c|}{ Maxwell's Equation } \\
\hline Laws & Differential Form & Integral Form \\
\hline 1-Gauss' Law & $\nabla \cdot E=\frac{\rho_{v}}{\varepsilon_{0}}$ & $\oint E \cdot d S=\int \frac{\rho_{v}}{\varepsilon_{0}} d V$ \\
\hline 2-Gauss' Magnetism Law & $\nabla \cdot H=0$ & $\oint H \cdot d S=0$ \\
\hline 3-Faraday's Law & $\nabla \times E=-\mu_{0} \frac{\partial \mathrm{H}}{\partial \mathrm{t}}$ & $\oint E \cdot d l=\int\left(-\mu_{0} \frac{\partial \mathrm{H}}{\partial \mathrm{t}}\right) \cdot d S$ \\
\hline 4-Ampère-Maxwell's Law & $\nabla \times H=J+\varepsilon_{0} \frac{\partial \mathrm{E}}{\partial \mathrm{t}}$ & $\oint H \cdot d l=\int\left(J+\varepsilon_{0} \frac{\partial \mathrm{E}}{\partial \mathrm{t}}\right) \cdot d S$ \\
\hline
\end{tabular}

In addition to Faraday's and Ampere's Law ( $3 \& 4)$, Gauss also contributed to the field of electromagnetics (Laws $1 \&$ 2). The first law describes the behavior of electric fields around electric charges. It states that the total electric flux out of a closed surface is proportional to the enclosed charge. Convention establishes that positive charges act as 
sources for electric fields and negative charges as sinks. Gauss' law on magnetism, however, dictates that magnetic monopoles do not exist! Unlike electric charges that can be isolated, every magnetic object is a magnetic dipole with a north and south pole. Despite the many intellectual insights and discoveries due to Maxwell's equations, there still remain many unanswered questions, particularly those concerning ferromagnetism. The field of quantum mechanics helps bridge many gaps of knowledge in the field of magnetism, but that will be discussed in further detail later in this chapter.

It is also important to understand the concept of magnetic moment. This can be understood in terms of magnetic poles and currents. If a bar magnet is at an angle with a magnetic field, it experiences a torque. This torque is expressed as: $\tau=p H l \sin \theta$ where $\boldsymbol{p}$ is the pole strength, $\boldsymbol{l}$ is the length of the magnet, and $\boldsymbol{\theta}$ the angle the field makes with the magnet. The magnetic moment of the magnet is defined as: $m=p l$. This quantity is of upmost importance since it can easily be measured with precision, whereas $p$ and $l$ are difficult to measure and quantify individually. The expression of torque can be further simplified to: $\tau=m \times H$. In terms of a current loop with an area $\boldsymbol{A}$ and a current $\boldsymbol{I}$, the torque is: $\tau=A I H \sin \theta$ where the magnetic moment is: $m=I A$. A magnet that isn't parallel to a magnetic field has a potential energy relative to the parallel position. The work done in turning the magnet is:

$$
E=\int \tau d \theta=\int m H \sin \theta d \theta=-m H \cos \theta=-m \cdot H
$$

This same equation can be used to describe the energy of a magnetic dipole at an angle $\theta$ with respect to the magnetic field. In a magnetic dipole, the magnetic moment is finite but the length of a magnet (or the area of a current loop) approaches zero. We can 
visualize any magnet as a sum of its individual dipole moments. Understanding the concepts of magnetic moment, magnetic dipole, and the work involved in rotating a magnet in the presence of a field is important because they will appear repeatedly when I introduce more detailed concepts in magnetism.

Maxwell's second law dictates that there can't exist any magnetic monopoles. No matter how many times we break magnets down into pieces, new magnetic dipoles emerge. We know that the magnetic moment $m$ is dependent on both the pole strength $p$ and length $l$, so we can introduce a concept that describes the distribution of magnetic moments in a material. The magnetization $M$ is the magnetic moment per unit volume: $M=\frac{m}{V}$. This property of the material depends on the collection of magnetic dipoles and how they interact with each other. When a magnetic field is applied to a material it undergoes magnetic induction $\boldsymbol{B}$, where $B=H+4 \pi M$ (CGS units) or $B=\mu_{0}(H+M)$ (SI units). Here it begins to become obvious the confusion and challenges of one unit system versus the other.

Magnetic induction is the density of flux inside a material: $B=\frac{\Phi}{A}$. Materials can be classified according to their level of flux inside $(B=H+4 \pi M)$ compared with the outside $(B=H)$ when a magnetic field is applied. Materials with less flux inside compared to the outside are diamagnetic. Should they have slightly more flux they can either be paramagnetic or antiferromagnetic. If they exhibit much greater flux, then materials can be classified as either ferromagnetic or ferrimagnetic. To further distinguish magnetic materials, measurements need to be done with varying temperatures. More details on the classification of materials will be discussed later. 


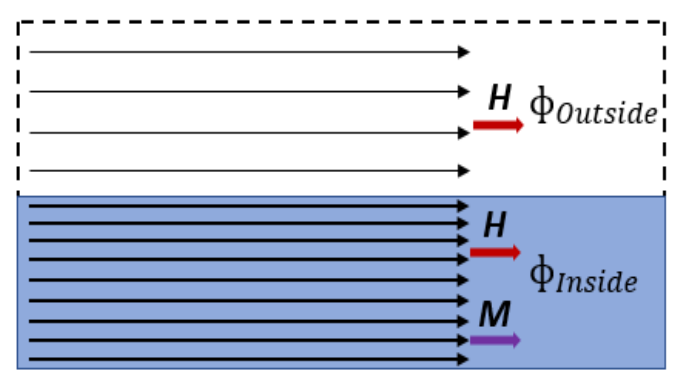

$$
\begin{aligned}
& \phi_{\text {Inside }}<\phi_{\text {Outside }} \rightarrow \text { Diamagnetic } \\
& \phi_{\text {Inside }}>\phi_{\text {Outside }} \rightarrow \text { Paramagnetic or Antiferromagnetic } \\
& \phi_{\text {Inside }}>>\phi_{\text {Outside }} \rightarrow \text { Ferromagnetic or Ferrimagnetic }
\end{aligned}
$$

Figure 2-3: Flux Density In and Out of a Material

The amount of magnetization depends on the amount of magnetic field $H$ applied. The rate at which a material is magnetized with respect to $H$ is known as the susceptibility $\chi$. The susceptibility is a dimensionless proportionality that describes the ease at which a material is magnetized and is defined as the ratio between the magnetization and the applied field: $\chi=\frac{M}{H}$. In a similar fashion, we can also relate magnetic induction B with the applied field. Permeability $\boldsymbol{\mu}$ is a quantity that also describes the ability of a material to form a magnetic field within itself. It is also defined as the ratio between magnetic induction and the applied magnetic field: $\mu=\frac{B}{H}$. Ferromagnets, for example, have high flux density and therefore high permeability. Since $\frac{B}{H}=1+4 \pi\left(\frac{M}{H}\right)$, the permeability and the susceptibility are related by: $\mu=1+4 \pi \chi$. In free space $\chi=0$ and $\mu=1$ since there is nothing to magnetize. Diamagnetic materials have a small but negative $\chi$, and $\mu$ is slightly less than 1. Paramagnetic and antiferromagnetic materials have a small and positive $\chi$, and $\mu$ is slightly greater than 1 . Ferromagnetic and ferrimagnetic materials have a large and positive $\chi$, and $\mu$ is much greater than 1 . Furthermore, the susceptibility and permeability in ferromagnets and ferrimagnets are also a function of the applied field $H$. They have a non-linear relationship, so their values vary are in accordance to the flux measured in these materials when a magnetic field is applied. 
$\mathrm{M}$ vs. $\mathrm{H}$ and $\mathrm{B}$ vs. $\mathrm{H}$ graphs are magnetization curves that are useful for determining magnetic properties. There are small changes in magnetization even when high fields are used for diamagnetic, paramagnetic, and antiferromagnetic materials. The magnetization is zero in these materials the instant the applied field is removed. Ferro- and ferrimagnets, on the other hand, obtain large values of magnetization with just a small applied field. This magnetization also saturates above a certain applied field. These materials exhibit hysteresis, meaning that removing or decreasing the applied field doesn't necessarily bring the magnetization to zero.
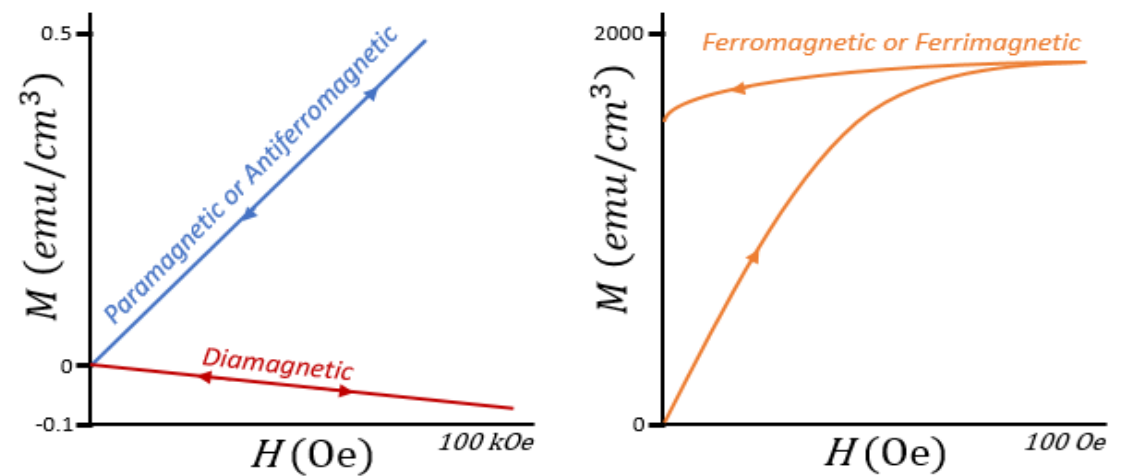

Figure 2-4: Magnetization Curves for Different Materials

Ferromagnetic and ferrimagnetic magnetization curves show that with enough field, the magnetization will saturate $\left(\mathrm{B}_{\mathrm{S}}\right.$ or $\left.\mathrm{M}_{\mathrm{S}}\right)$ and become constant. If only a small field is needed to saturate the magnet, it is said to be magnetically soft. Should the material require high fields to saturate, it is magnetically hard. The magnetic hardness isn't necessarily an intrinsic property since the same material can be both soft and hard depending on its physical condition. After saturation, the magnetic induction increases linearly with small gains like a paramagnet. If saturation occurs and the field is brought to 0 , the induction decreases from saturation $B_{S}\left(\right.$ or $\left.M_{S}\right)$ to retention $B_{R}\left(\right.$ or $\left.M_{R}\right)$. The reversing field required to reduce the magnetization to 0 is called the coercivity field 
$\left(\mathrm{H}_{\mathrm{C}}\right)$. Magnetic materials with coercivities around $\sim 100$ Oe are considered soft, and those greater than 5000 Oe are hard. Saturation can be obtained in the reverse direction also ($B_{S}$ or $-M_{S}$ ). A B-H (or M-H) loop that traces from positive saturation to negative and back to positive is known as a major hysteresis loop. Even if saturation isn't achieved, retention is still possible, mapping a minor hysteresis loop. While there can only be one major loop, there are an infinite number of minor loops possible.
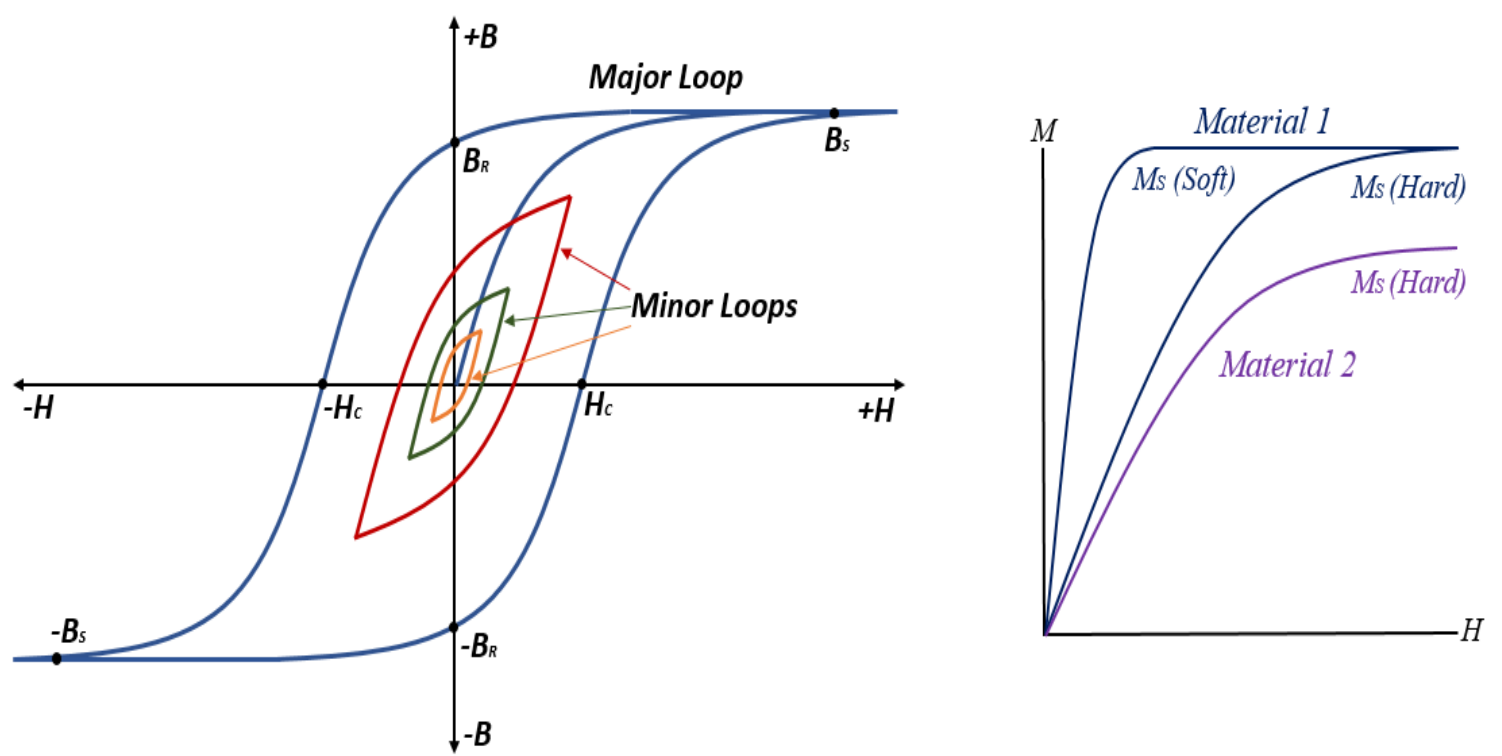

Figure 2-5: Major and Minor Loops

Before we go further, it's important to address the two major systems of units used in magnetism: The International System (SI) and Gaussian (CGS). While both describe magnetism appropriately, the units reflect the way it is visualized [17]. Converting between units is not always straightforward and can involve more than just multiplying a numerical factor. The following table shows some of the more important magnetic terms and their conversion: 
Table 2-2: CGS and SI units and Conversion

\begin{tabular}{|l|l|l|l|}
\hline Magnetic Term & CGS Units & SI Units & Conversion \\
\hline Magnetic Field $(H)$ & Oersted $(\mathrm{Oe})$ & $\mathrm{A} / \mathrm{m}$ & $1 \mathrm{Oe}=1000 / 4 \pi \mathrm{A} / \mathrm{m}$ \\
\hline Magnetic Flux $(\phi)$ & Maxwell $(\mathrm{Mx})$ & Weber $(\mathrm{Wb})$ & \\
\hline Magnetic Flux Density $(B)$ & Gauss $(\mathrm{G})$ & Tesla $(\mathrm{T})$ & $10^{4} \mathrm{G}=1 \mathrm{~T}$ \\
\hline Magnetic Moment $(m)$ & $\mathrm{emu}$ & $\mathrm{Am}^{2}$ & $10^{3} \mathrm{emu}=1 \mathrm{Am}^{2}$ \\
\hline Magnetization $(M)$ & emu/cm & $\mathrm{A} / \mathrm{m}$ & $10^{3} \mathrm{emu} / \mathrm{cm}^{3}=1 \mathrm{Am}$ \\
\hline Susceptibility $(\chi)$ & dimensionless & dimensionless & $1(\mathrm{CGS})=4 \pi(\mathrm{SI})$ \\
\hline Permeability $(\mu)$ & dimensionless & H/m & $1(\mathrm{CGS})=4 \pi^{*} 10^{-7} \mathrm{H} / \mathrm{m}$ \\
\hline
\end{tabular}

\subsubsection{Magnetism of Electrons}

Ampère theorized that all magnetic effects are due to "molecular" current loops. The atoms that make up materials have magnetic moments [14]. Electrons are much bigger contributors of magnetic moment than protons and neutrons, attributed mostly to their motion. Electrons are small, negatively charged particles that possess angular momentum because of its orbital motion around a nucleus. This angular momentum contributes to its magnetic moment like a small current loop since its equivalent to a circulation of charge. Because we are dealing with electrons, some quantum mechanical terms will be reviewed because quantum mechanics govern the behavior of particles at the atomic level.

In addition to particle behavior, electrons also exhibit wave like properties. Schrodinger's equation describes a wave function that analytically dictates the behavior of particles. This equation is used to find allowable energy levels in a quantum mechanical system in addition to other behavioral aspects. It's important to understand that at the atomic level the angular momentum of electrons is also quantized. Both the magnitude and direction 
of the momentum are restricted to certain values. The quantum parameters $\boldsymbol{n}, \boldsymbol{l}$, and $\boldsymbol{m}_{\boldsymbol{l}}$ are found in the solution of the wave equation and have important physical interpretations.

The parameter $\boldsymbol{n}$ is a principal quantum number can only take positive integers $(1,2$, etc.) and determines the energy of the electron level. Electrons with a certain $n$ value form the nth electron shell, where the larger the $n$ the larger the orbital the electrons can be found in. This parameter is not magnetically impactful, but can affect the values of other parameters that are. The parameter $l$ is the orbital quantum number that describes the magnitude of the orbital angular momentum of an electron, and can take integer values from 0 to $n-1$. Depending on the $n$ value, different $l$ values describe different orbital shapes. For example, at $l=0$ the orbit takes the shape of a sphere (s-orbital) and $l=1$ takes the shape of a dumbbell (p-orbital). The magnitude of the orbital angular momentum $|L|$ of a single electron is: $|L|=\sqrt{l(l+1)} \hbar$, where $\hbar$ is Planck's reduced constant $\frac{h}{2 \pi}$ ( $h$ is Planck's constant). It is apparent that the $n$ parameter influences the magnitude of the angular momentum of an electron. Finally, $\boldsymbol{m}_{l}$ is the magnetic quantum number which describes the orientation of the orbital angular momentum with respect to a magnetic field. The available values it can take are from $-l$ to $+l$. Mathematically, the component of the orbital angular momentum along the field can never be greater than the total orbital angular momentum. This means that the vector can never fully point along the magnetic field and therefore precesses around it. In the macroscopic case, where $l$ is very large, the component along the field is apparently equal (but not quite!) to the total orbital angular momentum and therefore appears to point in the exact direction of the field. 


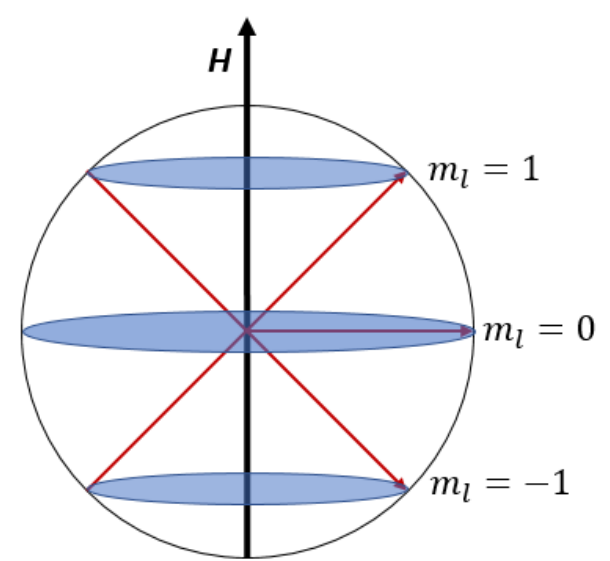

Figure 2-6: Component of Angular Momentum

Electrons orbit the nucleus so we can think of them as small current loops $[15,16]$. The angular momentum $L$ of any orbiting object is: $L=m v R$, where $\boldsymbol{R}$ is the distance of the orbiting electron from the nucleus, $\boldsymbol{m}$ is the mass of the orbiting object, and $\boldsymbol{v}$ is the velocity of the object. Since electrons are atomic particles, the orbital angular momentum is subject to quantization: $L=m v R=m_{l} \hbar$. If we consider the magnetic moment $\mu=I A$ (for atomic magnetic moments, $\boldsymbol{\mu}$ is typically used instead of $m$ ) and the quantization of the orbital angular momentum, we find that:

$$
\mu=(I)(A)=\left(\frac{e}{(2 \pi R / v)}\right)\left(\pi R^{2}\right)=m_{l}\left(\frac{e \hbar}{2 m_{e}}\right)
$$

The magnetic moment in the first Bohr orbit $\left(m_{l}=1\right)$ is: $\mu=9.27 \times 10^{-24} \frac{J}{T}$ (SI), a value that is a fundamental quantity and is referred to as the Bohr Magnetron $\left(\boldsymbol{\mu}_{\boldsymbol{B}}\right)$. We can express the magnetic moment as: $\mu=-\mu_{B} m_{l}$ (negative because of the electron charge). This value is the projected amount of magnetic moment on the field axis, however the magnitude of the total orbital magnetic moment is $\mu=\mu_{B} \sqrt{l(l+1)}$.

The potential energy relative to the field direction is defined as $E=-m \cdot H=\mu_{B} m_{l} H$. In the presence of a magnetic field, the energies split by an amount proportional to both 
the orbital angular momentum and the applied magnetic field. This is known as the normal Zeeman effect [18], an effect that has been observed in atomic absorption spectroscopy. For example, in the p-orbital, when a magnetic field is applied the energy is split into 3 since the magnetic quantum number $m_{l}$ can obtain values of $-1,0$, and 1 . On the other hand, s-orbitals will always have $m_{l}$ equal to 0 , and won't have any energy splitting.

The spin of an electron is another contributor to the total angular momentum of an electron. The classical interpretation of spin is the idea that the electron is a sphere spinning about its own axis. This is purely a visual aid with no quantitative substance. The concept of spin was utilized as a necessary solution to explain certain aspects of the Zeeman spectra. A quantum spin parameter was designated as $s$ and has the value of $\frac{1}{2}$. The magnitude of the spin angular momentum $|S|$ is given as:

$$
|S|=\sqrt{s(s+1)} \hbar=\frac{\sqrt{3}}{2} \hbar .
$$

Like $m_{l}$, spin also has an orientation $m_{S}$ because the spin angular momentum is also quantized. This quantum parameter can only take values $-\frac{1}{2}$ and $+\frac{1}{2}$. The spin angular momentum along the field is then given as $m_{S} \hbar= \pm \frac{\hbar}{2}$. Like the orbital angular momentum, the spin angular momentum vector can't point directly along the line of the applied field and therefore precesses around it also. Quantum mechanics dictate that the equation for spin magnetic moment along the field is: $\mu=-g_{e} \mu_{B} m_{s}$, and the magnitude of the spin magnetic moment is: $\mu=g_{e} \mu_{B} \sqrt{s(s+1)}$. Where $g_{e}$ is the g-factor of an electron. The g-factor in this context is $\sim 2$, so the spin magnetic moment along the field is $1 \mu_{B}$. 
So far, these calculations only consider a single electron. In an individual atom (besides hydrogen) there are more than one electron that interact with each other and the nucleus. These additional interactions are an analytical nightmare! What is known, however, is that electrons with lower angular momentum have lower energy and therefore the order in which atomic orbitals are filled (1s, 2s, 2p, 3s, etc.) can be understood. Electrons will tend to occupy orbitals of lower energy first and work their way up. As the electrons fill these orbitals, they shield each other from the Coulombic attraction to the nucleus. It is clear from the order in which orbitals are filled that electrons with lower magnetic moment $(l)$ are closer to the nucleus. It is also important to understand that this ordering also depends on the Pauli exclusion principle, where no 2 electrons can share the exact same quantum numbers. Each atomic orbital can be occupied by up to 2 electrons with opposite spins.

It is established that an electron has magnetic moment due to both its orbit and spin. There is also an interaction between both, called spin-orbit coupling. Spin-orbit coupling describes that the larger the atom (greater $\mathrm{Z}$ ), the larger the spin-orbit interaction. Furthermore, there are interactions within spins (spin-spin coupling) and orbits (orbitorbit interaction). The magnetic moment of an atom is determined by the comprehensive sum of all these interactions. To calculate the total angular momentum of all the electrons in an atom is complicated and depends on the $l$ and $s$ quantum numbers of each electron. Depending on the size of the atom different methods are used to calculate the total angular momentum.

Atoms with a small $\mathrm{Z}$, such as first row transition series, have weak spin-orbit interactions (ignored) so coupling within the individual spins and the orbits are dominant 
[14]. Individual summations of the orbital momentum for a total amount $L$ and spin momentum $S$ are done. The allowed values for $L$ is given by the Clebsch-Gordon series and $S$ are simply found by adding individual $m_{S}$ together. We can define a magnetic quantum number $\boldsymbol{M}_{L}$ and $\boldsymbol{M}_{S}$ (like $m_{l}$ and $m_{S}$ ) which describes the orientation of the total orbital angular momentum and spin momentum with respect to a magnetic field. $M_{L}$ takes values from $-L,-L+1, \ldots,+L$ and $M_{S}$ takes values from $-S,-S+1, \ldots,+S$. The total angular momentum $\boldsymbol{J}$ is simply the vector sum of both the total orbital momentum and the spin momentum: $J=L+S, L+S-1, \ldots,|L-S|$. The magnitude of the spin angular momentum: $|J|=\sqrt{J(J+1)} \hbar$ and the magnetic orientations $\boldsymbol{M}_{J}$ are from $-J,-J+1, \ldots,+J$. The total angular momentum component along the field is then: $M_{J} \hbar$. This approximation for light atoms is known as LS coupling or Russell-Saunders coupling. To summarize, the interactions between the spin and the orbit are weak so electron orbital angular momenta interact among themselves forming a total orbital angular momentum $L$, and the same with spins that also form a total spin angular momentum $S$. Together $L$ and $S$ form a total angular momentum $J$. The electrons needed for these calculations are only those with incomplete outer shells.

For heavy atoms (large Z), there is a strong spin-orbit interaction [14]. This interaction is typically larger than spin-spin and orbit-orbit interactions (ignored). Each electron has a resultant total angular momentum: $j_{i}=l_{i}+s_{i}$, where the total angular momentum of the atom is: $J=\sum j_{i}$. This coupling configuration is known as $j j$-coupling. Filled shells yield zero magnetic angular momentum and therefore cannot contribute to the magnetic dipole moment of the atom. 
Electrons want to occupy remain in the lowest energy configuration. Friedrich Hund defined three rules for identifying this configuration. His assumptions are based on the angular momentum calculated by $L S$-coupling (so they aren't applicable to heavy atoms) [19]. His first rule is based on the Pauli exclusion principle, where each orbit can only be occupied by at most two electrons with opposite spin. This rule states that the lowest energy configuration is achieved by maximizing the total spin angular momentum $S$ in the orbital shell. Basically, spins will first align themselves in parallel before pairing up in an antiparallel state. This makes physical sense since parallel spins are more likely to avoid each other, resulting in less Coulombic interaction (less energy). His second rule dictates that electrons will align themselves to maximize the total orbital angular momentum $L$. Electrons interact less if orbiting in the same direction. His third rule states that electron shells that are less than half full are configured to minimize the total angular momentum $J$; and if more than half full, the maximum $J$. The third rule was made under the assumption that dipoles in an anti-parallel configuration exhibit lower energy than those that are parallel. With heavier metals jj-coupling takes precedence and Hund's rules become inapplicable.

The normal Zeeman effect mentioned before is for atoms that have $S=0$. The anomalous Zeeman effect show much more spectral lines due to spin-orbit coupling [20]. With an applied magnetic field, there are multiple projections of the total magnetic angular momentum $M_{J}$, generating more spectral lines. The unequal splitting of the upper and lower levels of the transition further complicates this matter. This inequality is due to the g-factor of the electron. The total atomic magnetic moment along the field axis is a function of $S$ and $L$ in addition to $J$. The total magnetic moment can be expressed as: 
$\mu=-g \mu_{B} M_{J}$ and the magnitude as $\mu=-g \mu_{B} \sqrt{J(J+1)}$ where $g$ is called the Landé g-factor:

$$
g=1+\frac{J(J+1)+S(S+1)-L(L+1)}{2 J(J+1)}
$$

When $S=0$ we find that $g=1$ and we observe a normal Zeeman effect with upper and lower energies split equally. If $S \neq 0$, the $g$-factor is a combination of both $L$ and $S$, so the levels are split unevenly. The g-factor is basically an interpolation of both the spin angular momentum and the orbital angular momentum of an atom. The number reflects the amount that the spin and orbit contribute to the total magnetic momentum. Should the applied magnetic field be too strong, the coupling between S and L is broken and they will directly couple and precess around the applied field. This effect is known as the Paschen-Back effect.

The amount of magnetic moment is directly proportional to the value of the g-factor. Unfortunately, the magnetic moment calculated isn't always consistent with the experimental values. Crystal lattices couple strongly with the orbit. In many cases orbits find themselves fixed because of electric fields generated by the ions that surround them. This effect is known as quenching of the orbital angular momentum. Quenched orbits resist the motion to orient themselves along the applied field, so they do not contribute to the magnetic moment. Spins, however, don't interact strongly with the lattice and therefore become sole contributors to the magnetic moment. In first row transition metals the experimental values of magnetic moment closely match the moments calculated with only spin angular momentum. Spin only contribution changes the value of the previously calculated g-factor. 
The purpose of this sub-section was to explore the origin of magnetic dipoles at the atomic level. Magnetic materials are made of atoms, and understanding their magnetic behavior at the fundamental level will provide insight in the behaviors when they are grouped together to form thin films, particles, and bulk material. The electronic structure of a material is a meaningful aspect to classifying materials.

\subsubsection{Classification of Magnetic Materials}

\section{Diamagnetism}

The diamagnetic effect is the change of the orbital motion of an electron when an external magnetic field is applied. This effect is present in all atoms and it's so weak that it is hard to detect and is usually overshadowed by other interactions such as paramagnetism and ferromagnetism. Diamagnetism is only dominant in atoms with zero net magnetic moment. These atoms usually have electron shells that are full.

Diamagnetic materials reduce magnetic flux when a field is applied. Lenz's law relates to this effect, because an applied field will generate currents in the opposite direction in the atom via induction (Faraday's law). These currents generate fields in the opposite direction of the applied field. The stronger the applied field, the stronger the opposing fields. Noble gases (ex: Ar) are all diamagnetic because of their complete electron shells, and many diatomic gases $\left(\mathrm{ex}: \mathrm{H}_{2}\right)$ are also since electrons pair up in the molecular orbitals, reducing the magnetic moment to zero. There is not much of a temperature dependence in the diamagnetic effect. Some ionic solids (ex: $\mathrm{NaCl}$ ), materials with covalent bonds (ex: $\mathrm{Si}$ ), some metals $(\mathrm{Au})$, and organic compounds are also found to be diamagnetic. Susceptibility is very small and negative in these materials, typically in the order of $10^{-6}$ per unit volume. 
Superconductors are the most popular known materials that exhibit diamagnetism. These materials below a critical temperature will go from a conductor with a certain amount of electrical resistivity to having zero resistivity. In the superconducting state, they have a susceptibility of -1 due to macroscopic currents that circulate in the material suppressing the magnetic flux completely. This perfect diamagnetism is known as the Meissner effect. In superconductors there is however a high enough magnetic field, called the critical field that will nullify the superconducting state. The lower the temperature, the higher the critical field. Superconductors can generate high magnetic fields and have many practical applications.

\section{Paramagnetism}

Paramagnetic effects occur in materials that have a net magnetic moment. These magnetic moments, however, are weakly coupled to each other so thermal effects randomly align them. When a magnetic field is applied, they begin to align themselves in the direction of the applied field. This effect is usually observed in materials that have a net magnetic moment due to unpaired electrons in partially filled orbitals. These materials include certain metals (ex: $\mathrm{Al})$, some diatomic gases $\left(\mathrm{O}_{2}\right)$, rare earth and transition metal ions, salts, and oxides. Susceptibility is small and positive, between $10^{-3}$ and $10^{-5}$ per unit volume.

Due to thermal energy, there is a temperature dependence that creates an inverse relation to how well the moments align to the applied field. Langevin developed a theory that relates the temperature with the susceptibility by assuming that the magnetic moments in a material are non-interacting and that their orientation is function of thermal energy and

the field applied. Boltzmann statistics $\boldsymbol{e}^{-\boldsymbol{E} / \boldsymbol{k} \boldsymbol{T}}$ are used to describe an average distribution 
of the orientation of the magnetic moments. Considering a unit volume containing $\boldsymbol{n}$ amount of moments, we envision a sphere where all the moment vectors are drawn from the center. The number $d n$ of moments between $\theta$ and $\theta+d \theta$ is proportional to the surface area $d A$ of the sphere which they through:

$$
d n=K d A e^{-E / k T}=K 2 \pi R \sin \theta d \theta e^{\mu H \cos \theta / k T}
$$

$\boldsymbol{K}$ is a proportionality factor, $\boldsymbol{T}$ is the temperature, $\boldsymbol{R}$ is the radius of the sphere, and $\boldsymbol{k}$ is the Boltzmann factor. For simplification, we make $a=\mu H / k T$. The total number of moments is simply: $n=\int_{0}^{n} d n$ and we modify it in terms of $\theta$ so:

$$
n=2 \pi R K \int_{0}^{\pi} e^{a \cos \theta} \sin \theta d \theta
$$

The magnetization in the unit volume would just be the total amount of magnetic moments:

$$
\begin{gathered}
M=\int_{0}^{n} \mu \cos \theta d n=2 \pi \mu R K \int_{0}^{\pi} e^{a \cos \theta} \sin \theta \cos \theta d \theta=\frac{n 2 \pi \mu R K \int_{0}^{\pi} e^{a \cos \theta} \sin \theta \cos \theta d \theta}{2 \pi R K \int_{0}^{\pi} e^{a \cos \theta} \sin \theta \cos \theta d \theta} \\
=\frac{n \mu \int_{0}^{\pi} e^{a \cos \theta} \sin \theta \cos \theta d \theta}{\int_{0}^{\pi} e^{a \cos \theta} \sin \theta \cos \theta d \theta}=n \mu\left(\operatorname{coth} a-\frac{1}{a}\right)
\end{gathered}
$$

The maximum magnetization $\left(\mathrm{M}_{0}\right)$ would occur if all the moments were perfectly aligned with the field: $M_{0}=n \mu$. The evaluated integral can be reduced to: $\frac{M}{M_{0}}=\operatorname{coth} a-$ $\frac{1}{a}$, an expression known as the Langevin function $\mathbf{L}(\boldsymbol{a})$. 


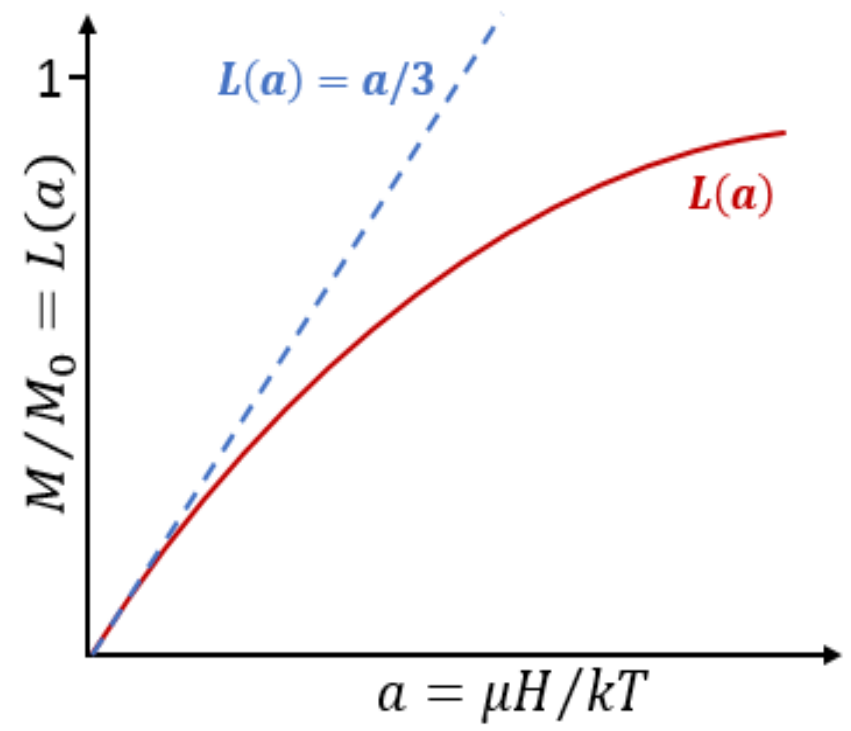

Figure 2-7: Langevin Curve

The Langevin function can be expanded as a Taylor series: $L(a)=\frac{a}{3}-\frac{a^{3}}{45}+\frac{2 a^{5}}{945}-\cdots$ where the first term will be considered since it is the most dominant for most realistic temperatures and magnetic fields:

$$
M=\frac{M_{0} a}{3}=\frac{n \mu a}{3}=\frac{n u^{2} H}{3 k T}
$$

and the susceptibility is:

$$
\chi=\frac{M}{H}=\frac{n u^{2}}{3 k T}=\frac{C}{T}
$$

$\boldsymbol{C}$ is called the Curie constant. This expression is known as Curie's Law and the susceptibility in paramagnets is inversely proportional to the temperature.

Quantum mechanics, however, limits the amount of available states the magnetic moment can take. Taking account quantization where energy $E=-g M_{j} \mu_{B} H$, the Boltzmann statistics $e^{-E / k T}=e^{g M_{j} \mu_{B} H / k T}$. The magnetization is the amount of moments times the average magnetic moment: 


$$
M=\frac{n \sum M_{j} \mu_{B} e^{g M_{j} \mu_{B} H / k T}}{\sum e^{g M_{j} \mu_{B} H / k T}}
$$

$M_{j}$ goes from $-J$ to $+J$ and $a^{\prime}=g J \mu_{B} H / k T$, the magnetization can be reduced to:

$$
M=n g J \mu_{B}\left[\frac{2 J+1}{2 J} \operatorname{coth}\left(\frac{2 J+1}{2 J}\right) a^{\prime}-\frac{1}{2 J} \operatorname{coth} \frac{a^{\prime}}{2 J}\right]
$$

The maximum magnetization $\left(M_{0}\right)$ occurs if all the magnetic moments were aligned with the field:

$$
M_{0}=n g J \mu_{B} \rightarrow \frac{M}{M_{0}}=\left[\frac{2 J+1}{2 J} \operatorname{coth}\left(\frac{2 J+1}{2 J}\right) a^{\prime}-\frac{1}{2 J} \operatorname{coth} \frac{a^{\prime}}{2 J}\right]
$$

This expression is called the Brillouin function $B\left(J, a^{\prime}\right)$. Should we take the limit as $J$ approaches $\infty$ we find that it yields the Langevin function. The Brillouin function can also be expanded as a Taylor series:

$$
B\left(J, a^{\prime}\right)=\frac{(J+1) a^{\prime}}{3 J}-\frac{\left[(J+1)^{2}+J^{2}\right](J+1)\left(a^{\prime}\right)^{3}}{90 J^{3}}+\cdots
$$

We take the first term as the relevant one and the susceptibility is:

$$
\chi=\frac{J(J+1) n g^{2} \mu_{B}^{2}}{3 k T}=\frac{C}{T}
$$

In both the Langevin and Brillouin functions we see that at low temperatures and high fields they approach 1, meaning the magnetization saturates. This makes intuitive sense because magnetic moments tend to align themselves with the magnetic field and thermal energy will cause them to fluctuate. Curie's law assumes no interaction between atomic magnetic moments, where they reorient themselves with an applied field independently. This, however, isn't always the case because there is evidence that some materials undergo some spontaneous ordering. Weiss assumed an interaction between the atomic 
moments or a "molecular field." This field is fictitious, but does account for the behavior of many paramagnets. Weiss assumed that the molecular field was proportional to the magnetization: $H_{m}=\gamma M$ where $\gamma$ is the molecular field constant, a material dependent coefficient. The total field acting on the material must be: $H_{T}=H+H_{m}$. The susceptibility is then:

$$
\chi=\frac{M}{H_{T}}=\frac{M}{H+H_{m}}=\frac{M}{H+\gamma M}
$$

We also know that:

$$
\chi=\frac{C}{T} \rightarrow \chi=\frac{M}{H+\gamma M}=\frac{C}{T} \rightarrow M=\frac{C H}{T-C \gamma}
$$

If we make $\theta=C \gamma$ we find that $\chi=\frac{C}{T-\theta}$, an expression called Curie-Weiss law. If atomic moments do not interact with each other then, $\theta=0$ and we have Curie's law. Should $\theta$ be positive, there is a stronger tendency to align to the field, whereas if it is negative it opposes it. It is apparent with the Curie-Weiss law that the susceptibility diverges at $T=\theta$. This divergence corresponds to a transition where a paramagnet will begin to act as a ferromagnet. More details on this critical temperature will be discussed in the ferromagnetic sub-section

The drawback with the Langevin theory is that it considers that electrons are localized and that individual atomic moments do not interact with each other. Metals have high conductivity, so electrons are not confined but rather move around the lattice. Band theory is more appropriate to understanding their magnetic behavior. Electrons orbit around an atom and occupy discrete energy levels. According to the Pauli exclusion principle no two electrons can share the same set of quantum numbers. Each energy level 
can permit two electrons, but with opposite spin. When atoms come together, to form a solid for example, their outermost electrons begin to overlap. Electrons with the same configuration begin to approach each other, but the Pauli exclusion principle can't allow these identical electrons to share the same values. What occurs is a splitting of the bands, and the electron configuration is altered. Electrons will then begin to fill the bands from the lowest energy to the highest. At $\mathrm{T}=0 \mathrm{~K}$, the highest energy electrons occupy is called the Fermi level. The number of atoms that come together to form a solid corresponds to an equal amount of energy levels formed.

Paramagnetic materials have an equal amount of up and down spin electrons at the Fermi level. However, when a magnetic field is applied, the electrons with spins pointing against the field have lower energy than those pointing in the same direction (magnetic moments point in the opposite direction of the spin due to the electron's negative charge). Electrons with magnetic moments parallel with the field will shift down the energy band, and those with moments anti-parallel will shift up. Spin magnetic moment is $1 \mu_{B}$, so the electron energy shift would be $H \mu_{B}$. Electrons with moments antiparallel to the field will try to rotate. The only way they can align their moments with the field is if a vacant parallel-moment energy state is made available. Electrons with antiparallel moments closest to the Fermi level can be promoted to higher energy bands and re-orient themselves, creating magnetization. Once the field is removed the electrons go back to their original place. This effect is known as Pauli Paramagnetism. The free electron model is used to derive an expression for paramagnetic susceptibility in metals:

$$
\chi=\frac{M}{H}=\frac{\mu_{B}^{2}(N / V)}{E_{F}}
$$


$\boldsymbol{N}$ is the number of electrons, $\boldsymbol{E}_{\boldsymbol{F}}$ is the Fermi energy, and $\boldsymbol{V}$ is the volume of the material. The susceptibility according to the free electron model is independent of temperature.
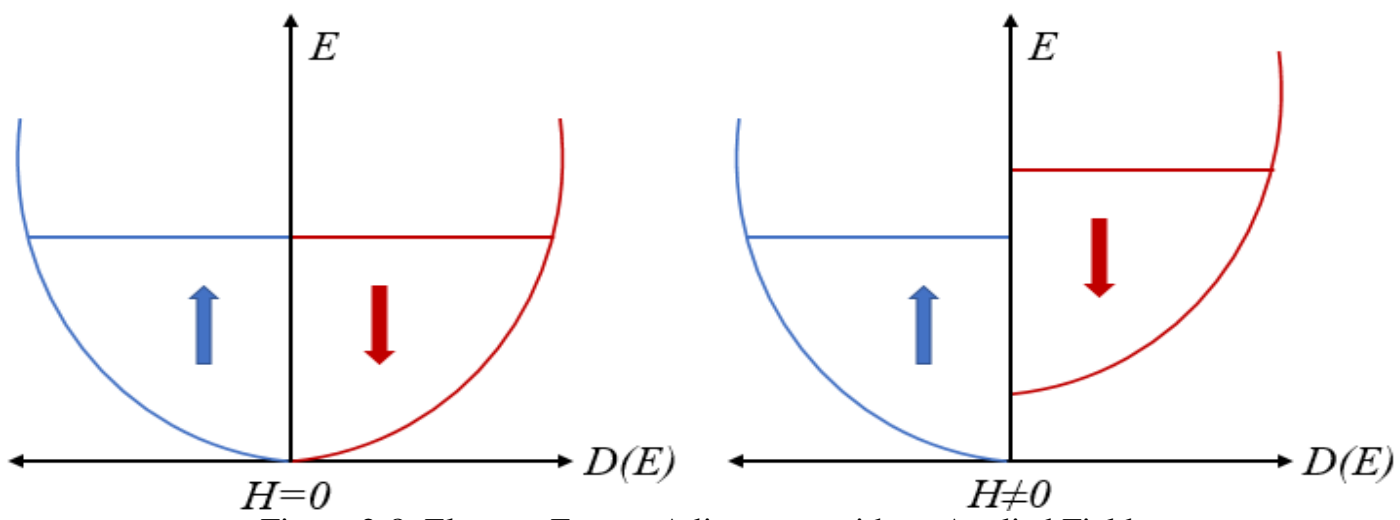

Figure 2-8: Electron Energy Adjustment with an Applied Field

\section{Ferromagnetism}

Ferromagnets can typically be brought to saturation with a relatively small applied magnetic field. With a similar field, the magnetization of a paramagnetic is typically millions of times less than that of a ferromagnet. Weiss was one of the first to develop a theory to understand this dramatic effect. With the Curie-Weiss law he figured that a strong intrinsic molecular field was responsible for magnetizing the material. He understood that temperatures above $\theta$ caused the material to become paramagnetic, and below ferromagnetic. Weiss argued that the molecular field was strong enough for the material to "self-saturate." Many times, however, we find that ferromagnets appear to be unmagnetized. Weiss responded to this problem by claiming that a ferromagnet consists of domains that are spontaneously magnetized in different directions. Applying a magnetic field orientate the domains in the same direction, hence creating a single domain magnetized in the direction of the applied field. 
Weiss's interpretation of ferromagnetism is known as molecular field theory. The critical temperature where $T-\theta=0$ is called the Curie temperature $T_{C}$. Below this temperature, the ferromagnet retains its spontaneous magnetization, above $T_{C}$ it becomes paramagnetic. Without the application of an external field, only the molecular field acts on it: $H_{T}=H_{m}=\gamma M$. If we plot the magnetization due to the molecular field and the Langevin function, we find that they intersect at 2 points: origin and a positive non-zero point. The point at the origin is magnetically unstable. A small field (ex: Earth's field) will magnetize it slightly, increasing its magnetization. This in turn will increase its molecular field and so on until it reaches the second point of intersection.

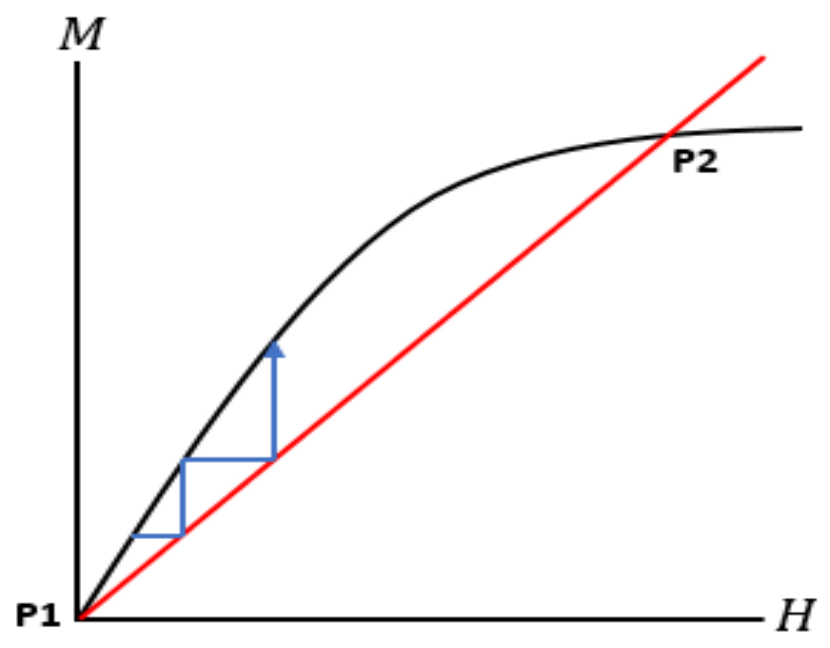

Figure 2-9: Spontaneous Magnetization

To understand the temperature dependency, we once again assume that the molecular field is the only magnetic field applied:

$$
a=\frac{\mu H_{M}}{k T}=\frac{\mu \gamma M}{k T} \rightarrow M=\left(\frac{k T}{\mu \gamma}\right) a
$$

With higher temperatures, the slope becomes steeper, resulting in the line intersecting at a lower spontaneous magnetization. To find the Curie temperature, we equate the first term of the Langevin function with the spontaneous magnetization field line: 


$$
M=\left(\frac{k T_{C}}{\mu \gamma}\right) a=\frac{n \mu a}{3} \rightarrow T_{C}=\frac{n \mu^{2} \gamma}{3 k}=\frac{\mu \gamma M_{0}}{3 k}
$$

Weiss's theory can be modernized to yield more accurate results by introducing quantization:

$$
a^{\prime}=\frac{g J \mu_{B} H_{M}}{k T}=\frac{g J \mu_{B} \gamma M}{k T} \rightarrow M=\left(\frac{k T}{g J \mu_{B} \gamma}\right) a^{\prime}
$$

To find the Curie temperature, we equate the first term of the Brillouin function with the spontaneous magnetization field line:

$$
M=\left(\frac{k T}{g J \mu_{B} \gamma}\right) a^{\prime}=\frac{(J+1) a^{\prime}}{3 J} \rightarrow T_{C}=\frac{(J+1) g \mu_{B} \gamma}{3 k}=\frac{(J+1) g \mu_{B} \gamma M_{0}}{3 k}
$$

The origin of the molecular field was explained by Heisenberg who claimed that it is due to quantum mechanical effects called exchange forces. When atoms are close, Coulomb's law accounts for electrostatic forces from the charges of the protons and electrons. However, there is a non-classical force that depends on the relative orientation of the spins, the exchange force. Spins with an antiparallel orientation can share the same orbit but will overlap spatially and result in stronger Coulombic repulsions. Parallel spins occupy different orbits so electrostatic repulsions are minimized. The term exchange revolves around the idea that two similar electrons can effectively "exchange" places if atoms are brought close enough together. The exchange energy between two atoms is given by:

$$
E_{e x}=-2 J_{e x} S_{i} S_{j}
$$

$S_{i}$ and $S_{j}$ are the spin angular momentums of both atoms $\boldsymbol{i}$ and $\boldsymbol{j}$, and $\boldsymbol{J}_{\boldsymbol{e} x}$ is the exchange integral. If the exchange integral is positive, spins must be parallel to reduce the overall energy. If it is negative, spins have an antiparallel configuration. For ferromagnetism to 
occur, the exchange integral must be positive. The Bethe-Slater curve relates the exchange integral with the interatomic distance. It shows that when atoms are close they tend to have a negative exchange integral and therefore an antiparallel spin configuration. The greater the separation, the exchange integral becomes positive and spins align themselves in parallel.

Weiss's molecular theory has been very accurate in determining the temperature dependence of the spontaneous magnetization and the transition to the paramagnetic state past the Curie temperature. Unfortunately, his theory does not accurately calculate the magnetic moment per atom in some materials, especially metals. Weiss assumed that the magnetic moment of each atom would be the same regardless if it was in the paramagnetic or ferromagnetic phase, which is experimentally proven to be incorrect. Dealing with localized ions where electrons do not interact with each other is not applicable to metals that have good conductivity.

The free electron model is used to understand ferromagnetism in metals. $\mathrm{Fe}, \mathrm{Co}$, and $\mathrm{Ni}$ are first row transition metals that exhibit ferromagnetic behavior. The outermost electrons are $3 \mathrm{~d}$ and $4 \mathrm{~s}$. When these elements approach each other, their electron clouds overlap and their electron levels begin to split and form bands. The Fermi level of these metals lies between the overlapped $3 \mathrm{~d}$ and $4 \mathrm{~s}$ bands. For simplification, we are going to operate under the assumption that the shape of the bands does not change for different elements. This assumption is known as the rigid-band model. Due to the overlap of the bands, valence electrons partially occupy both $3 \mathrm{~d}$ and $4 \mathrm{~s}$ bands. For example, Nickel has 10 valence electrons, where 9.4 occupy the $3 \mathrm{~d}$ band and 0.6 the $4 \mathrm{~s}$ band. The density of states of the $3 \mathrm{~d}$ band is greater since it can hold 10 electrons as opposed to 2 in the $4 \mathrm{~s}$ 
band. The energy required to promote a 4 s electron into a vacant state to flip its spin orientation is much higher than the decrease in the exchange energy. The $3 \mathrm{~d}$ band, however, has many electrons at the Fermi level. This band is also narrow and has plenty of closely packed energy levels that allow electrons to be easily promoted. This is a perfect recipe for the displacement of electrons due to exchange interactions, which result in spontaneous magnetization!

This model accounts for magnetization because of an unequal amount of spins. Band theory deals with a sea of electrons, where a probability distribution accounts for their location in the bands. Only $3 \mathrm{~d}$ electrons contribute to the magnetic moment because there is no exchange splitting in the $4 \mathrm{~s}$ band. This results in a non-integer value for magnetic moment, consistent with experiments. Choosing between the molecular field theory and the free electron model depends on the electrical properties of the material in question. A method that combines both models is the Density Function Theory (DFT). It accounts for all interactions between all electrons, and they are arranged to minimize the total energy. This theory requires computational software due to the intensity and amount of calculation needed.

\section{Antiferromagnetism}

Due to the small positive susceptibility, antiferromagnets are easily confused with paramagnets. When measuring the susceptibility versus temperature, above a critical temperature, the Néel temperature $\left(T_{N}\right)$, it is paramagnetic. Below $T_{N}$, the magnetic moments tend to align themselves in an antiparallel configuration. This magnetic alignment is formed in the absence of a magnetic field, but can become randomized (paramagnetic) with enough thermal energy. Two or more magnetic superlattices are 
formed with the magnetic moments pointing in opposite directions. The strength of the magnetic alignment increases by lowering the temperature, reducing its susceptibility.

As expected, the net magnetic moment in an antiferromagnet is zero. Like a ferromagnet, each magnetic lattice has spontaneous magnetization. Neutron diffraction is a useful technique to determine the magnetic ordering of a material. Neutrons are used because they have a magnetic moment, so they will scatter due to the magnetic moments of the electrons. Furthermore, they are electrically neutral, so they won't scatter due to charge. This type of diffraction is used to prove that magnetic ordering exists for certain materials below a critical temperature.

Many antiferromagnets are electrical insulators or semiconductors, so molecular field theory is an appropriate model to use. Assuming only 2 magnetic sublattices A and B, where $\mathrm{A}$ ions are closest to $\mathrm{B}$ ions. We ignore any interactions between like ions since they are farther apart, and focus only on the AB interaction. Two molecular fields must be considered: $H_{A}=-\gamma M_{B}$ and $H_{B}=-\gamma M_{A}$. Above $T_{N}$ we know it's paramagnetic, so Curie's law for susceptibility is:

$$
\chi=\frac{M}{H}=\frac{2 C^{\prime}}{T+C^{\prime} \gamma}=\left(\frac{C}{T-\theta}\right)
$$

$\theta$ is negative since the molecular fields tend to anti-align the magnetic moments. The Néel temperature occurs when the susceptibility diverges, $T_{N}=C^{\prime} \gamma$. Below the Néel temperature the susceptibility depends on the angle the external field makes with the spontaneous magnetization. With a field parallel with the magnetic sublattices, the sublattice where the magnetic moments are in the same direction as the field will increase in magnetization by the same amount that the sublattice that points in the opposite 
direction will diminish by. This change in magnetization is just the slope of the Brillouin function times the change in $a^{\prime}$. Understanding this we find that the susceptibility in the presence of a parallel field is:

$$
\chi_{\|}=\frac{2 N m^{2} B^{\prime}\left(J, a^{\prime}\right)}{2 k T+N m^{2} \gamma B^{\prime}\left(J, a^{\prime}\right)}
$$

$N$ is the number atoms per unit volume. At absolute zero we find that the susceptibility is zero since the sublattices are perfectly anti-aligned.

Should we apply a field perpendicular to magnetic moments of the sublattices, we rotate the magnetic moments magnetizing the sample in the same direction. At the same time, a molecular field will be set up to oppose this magnetization. Once equilibrium is reached, the external field is balanced by the molecular field, yielding a susceptibility of: $\chi_{\perp}=\frac{1}{\gamma}$, a constant value independent of temperature. Antiferromagnets have a greater perpendicular susceptibility than parallel.

Exchange forces are also responsible for the negative molecular field. The superexchange interaction is responsible for antiparallel configuration in many antiferromagnets. It is an interaction between neighboring cations through a non-magnetic anion. $\mathrm{MnO}$ is an antiferromagnet where the $\mathrm{d}$ orbitals from the $\mathrm{Mn}$ and the $\mathrm{p}$ orbitals from the $\mathrm{O}$ form a direct exchange. A manganese ion will covalently bond with oxygen. Oxygen has only two electrons it can donate with opposite spins (p-orbital). Oxygen will donate an electron with an up spin to a manganese ion with a down spin, and donate the other spindown electron to a manganese ion with an up spin. These $d$ and $p$ hybridized orbitals result in an antiparallel configuration of the magnetic moments between atoms. RKKY is another indirect exchange interaction theory [21] that results in an antiferromagnetic (and 
ferromagnetic) configuration. This interaction involves a magnetic ion that can polarize conduction electrons that in turn, can polarize surrounding magnetic ions. The distance between the magnetic ions affects whether the interaction is antiferromagnetic or ferromagnetic.

\section{Ferrimagnetism}

Ferrimagnets are very similar to ferromagnets because they have spontaneous magnetization below a critical temperature (Curie temperature $T_{C}$ ) and to antiferromagnets because they have antiparallel alignment of magnetic moments. Antiferromagnets have a net magnetization of 0 ; ferrimagnets on the other hand have a non-zero net magnetization. This is because the magnetization of one sublattice is greater than the other.

Weiss molecular theory is applicable to model ferrimagnetic behavior since most are ionic solids with localized electrons and low conduction. Because the sublattices are not structurally identical, we must consider three interactions: if we have sublattices A and B, the interactions are A-A, A-B, and B-B. The difference of sublattices is usually due to different ions or symmetry. We also assume that A-A and B-B interactions are ferromagnetic interactions and A-B antiferromagnetic, where the molecular fields are: $H_{A}=-\gamma_{A B} \beta M_{B}+\gamma_{A A} \alpha M_{A}$ and $H_{B}=-\gamma_{A B} \alpha M_{A}+\gamma_{B B} \beta M_{B}$. Néel proposed a model that made all the magnetic ions equal for both sublattices and instead used parameters $\alpha$ and $\beta$ are to account for the amount of magnetic moment that each sublattice contributes, where $\alpha+\beta=1$. The negative molecular field coefficients account for the antiferromagnetic interactions and the positive one for ferromagnetic interactions. 
Above $T_{C} M=M_{A}+M_{B}$ and we find the susceptibility is:

$$
\frac{1}{\chi}=\frac{T+C / \chi_{0}}{C}-\frac{b}{T-\theta}
$$

Where:

$$
\begin{gathered}
\frac{1}{\chi_{0}}=\gamma_{A B}\left(2 \alpha \beta-\frac{\gamma_{A A}}{\gamma_{A B}} \alpha^{2}-\frac{\gamma_{B B}}{\gamma_{A B}} \beta^{2}\right), b=\gamma_{A B}{ }^{2} C \alpha \beta\left[\alpha\left(1+\frac{\gamma_{A A}}{\gamma_{A B}}\right)-\beta\left(1+\frac{\gamma_{B B}}{\gamma_{A B}}\right)\right]^{2} \text { and } \\
\theta=\gamma_{A B} C \alpha \beta\left(2+\frac{\gamma_{A A}}{\gamma_{A B}}+\frac{\gamma_{B B}}{\gamma_{A B}}\right) .
\end{gathered}
$$

Below $T_{C}$ we find that the net magnetization is: $M=\left|M_{A}\right|-\left|M_{B}\right|$ where each sublattice follows the Brillouin function:

$$
M_{A}=N m_{A} B\left(J, \frac{m_{A} H_{A}}{k T}\right) \text { and } M_{B}=N m_{B} B\left(J, \frac{m_{B} H_{B}}{k T}\right)
$$

The parameters $\boldsymbol{m}_{\boldsymbol{A}}$ and $\boldsymbol{m}_{\boldsymbol{B}}$ are the magnetic moments of their respective ions along the direction of the field. Since $H_{A}$ depends on $M_{B}$ and $H_{B}$ depends on $M_{A}$, both of the magnetization equations are dependent of each other, requiring a numerically based solution. Both sublattices must have the same Curie temperature because a sublattice with zero moment would not be able to magnetize the other.

Ferrites are the most technologically relevant ferrimagnets. They are ferrimagnetic transition metal oxides. Many are electrical insulators so they found themselves useful in high frequency applications. Low conduction prevents eddy current induction when an AC field is applied, minimizing power losses. Ferrites are classified by their crystal structure: cubic ferrites and hexagonal ferrites. Cubic ferrites have the general formula: $\mathrm{MO} \cdot \mathrm{Fe}_{2} \mathrm{O}_{3}$, where $\mathrm{M}$ is a divalent metal ion, for example nickel ferrite: $\mathrm{NiO} \cdot \mathrm{Fe}_{2} \mathrm{O}_{3}$. Except for cobalt ferrite $\left(\mathrm{CoO} \cdot \mathrm{Fe}_{2} \mathrm{O}_{3}\right)$, they are all magnetically soft. Cubic ferrites have a spinel structure due to the similarity in crystal structure of the mineral spinel. The 
structure is complex, where the oxygen ions are arranged in a face-center cubic arrangement and the metal ions occupy the space between them in a tetrahedral and an octahedral arrangement. These two metal arrangements account for the magnetic sublattices deemed A and B sites. When the divalent metal ions occupy the A sites and the Fe ions occupy the B sites, their crystal structure is called normal spinel. When the divalent metal ions are on the $\mathrm{B}$ sites and Fe ions are equally divided between the $\mathrm{A}$ and B sites the crystal structure is called inverse spinel. Hexagonal ferrites are used as permanent magnets since they are magnetically hard and have high uniaxial crystal anisotropy. As the name implies, they have hexagonal crystal structures. The most important one is barium ferrite that has a hexagonal magnetoplumbite crystal structure. Other important ferrimagnets include garnets and maghemite.

\section{Summary}

The following figure visually summarizes the classification of magnetic materials.

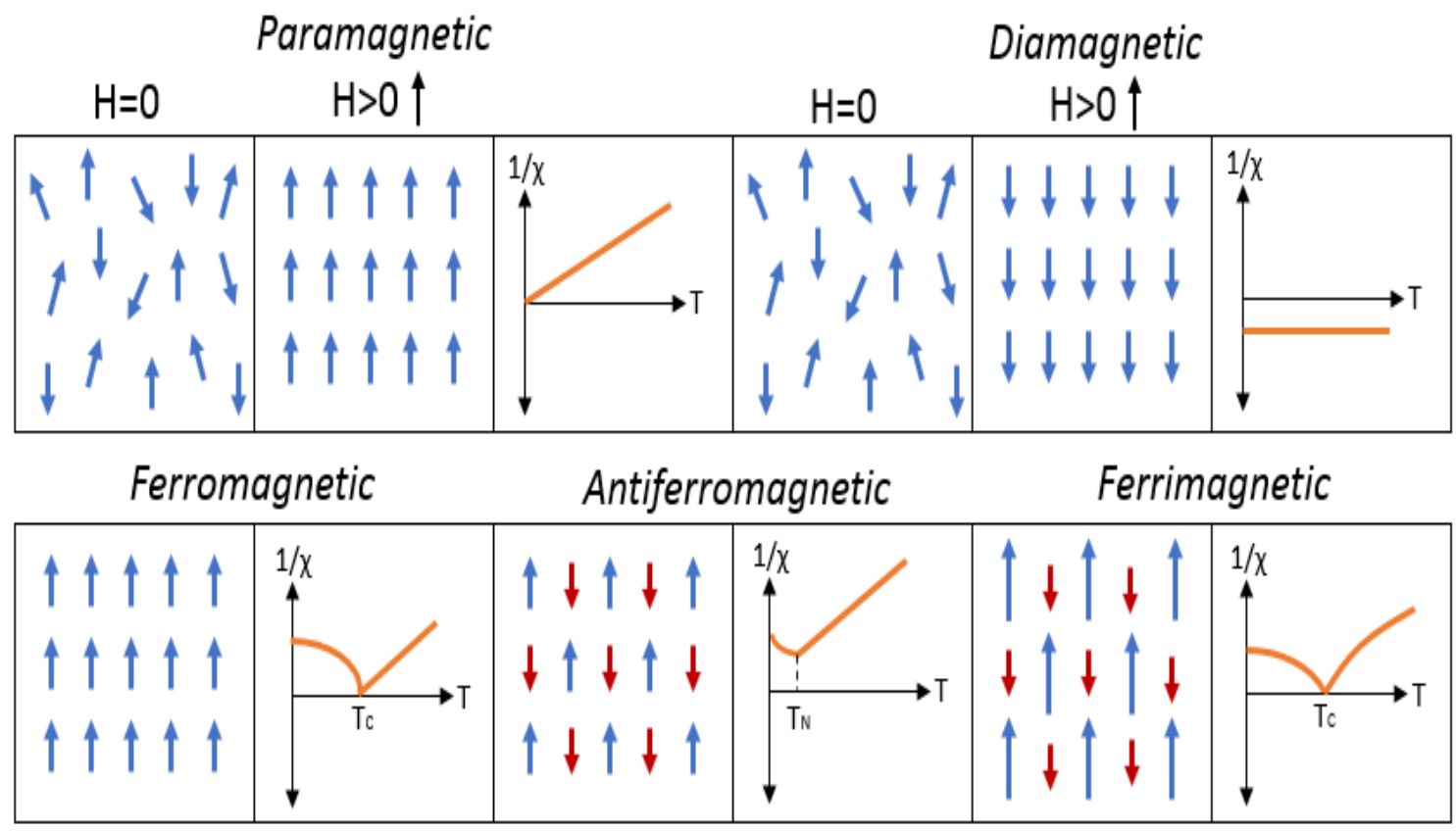

Figure 2-10: Magnetic Material Classification 


\subsection{Micromagnetism in Ferromagnets}

Magnetism is result of many competing processes. This section is dedicated to understanding ferromagnets further by analyzing them at the microscopic level. We know that ferromagnets have spontaneous magnetization, but we generally find them with a net magnetization of zero. This is due to the formations of ferromagnetic domains. When we apply a field, we rotate the domains so they point in the same direction, increasing the net magnetization of the ferromagnet. The section will cover the different physical processes that dictate the behavior of ferromagnets and the formation of domains.

Magnetic domains are microscopic and are separated from each other by domain walls. Exchange energies tend to align spins parallel to each other. A single domain would minimize the exchange energy, but there are other factors that come into play. These other factors include magnetostatics, magnetocrystalline anisotropy, magnetostriction, and Zeeman energy. The formation of magnetic domains stems from the ferromagnetic materials minimizing its energy.

\subsubsection{Magnetostatics}

Magnetostatic forces are a key factor in determining the magnetic microstructure. If we consider a sample of finite size, we find poles at the ends of it creating a magnetic field around it. This field acts against the magnetization of the sample. This demagnetizing field is responsible for magnetostatic energy and is dependent on the shape of the sample. This energy is minimized by reducing the demagnetization field by spitting the sample into domains. The sample will divide into enough domains removing any magnetic poles at the surface of the sample. This reduces the stray fields, minimizing the magnetostatic energy. Domains, however, prefer not to align themselves antiparallel to each other. This 
results in an increase of the exchange energy since at the domain boundaries the magnetic moments aren't parallel. Furthermore, the direction of magnetization also depends on the shape of the magnet since there is a preference to magnetize along the long axis over the short one. Perfect spheres will magnetize in any direction with equal preference (isotropic).
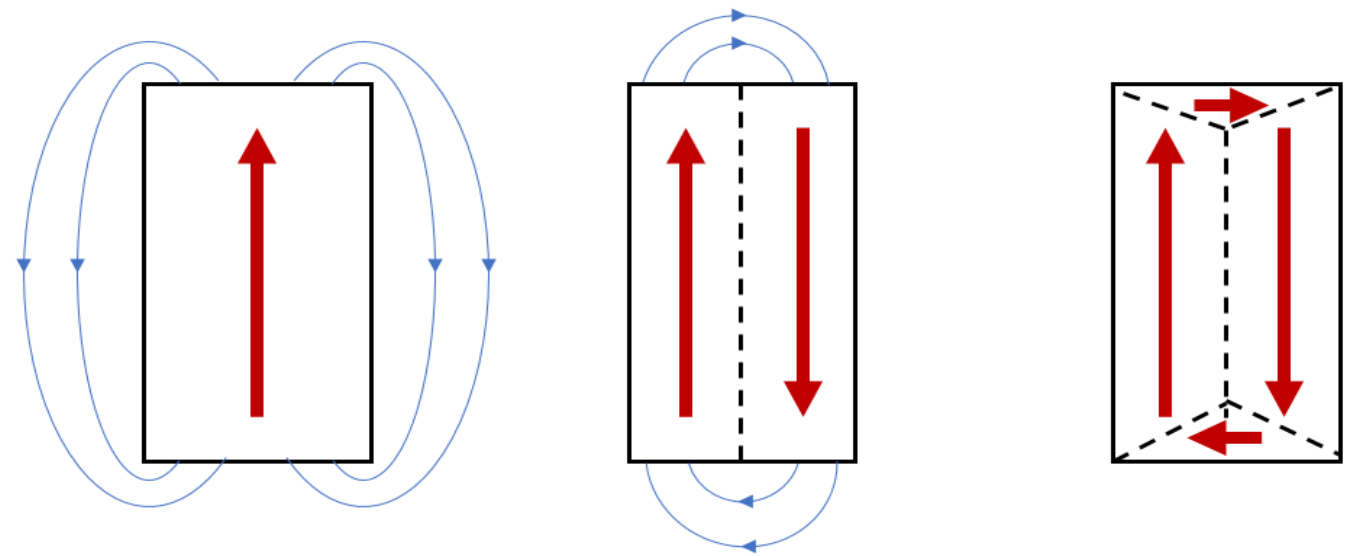

Figure 2-11: Domain Formations

\subsubsection{Magnetic Anisotropy}

The tendency for magnetic moments to align in a preferred crystallographic direction is known as magnetocrystalline anisotropy. The preferred direction is the one that saturates the easiest with an applied magnetic field, or "easy" axis. The direction where it is harder to magnetize and requires a larger field to saturate is called the "hard" axis. For example, in BCC iron, the easy axis is the [100] direction and the hard axis is [010] direction. The sample is at a higher energy if aligned along the hard axis instead of the easy axis. Domains will form to point along the easy axis. BCC iron has an easy axis in the 100 and due to cubic symmetry, domains will form in both the horizontal and vertical axis. Magnetocrystalline energy prefers large domains to avoid any domains in the hard axis. Magnetocrystalline anisotropy energy is defined as the energy difference per unit volume 
between the easy and hard directions. The energy to rotate spins away from the easy axis is the amount required to overcome spin-orbit coupling. If there is strong orbit-lattice interaction, this rotation is met with resistance. Materials with weak spin-orbit interaction will have small magnetocrystalline anisotropy. Rare-earth metals, for example, are heavy elements and therefore have strong spin-orbit coupling, creating strong anisotropy. This makes them useful as permanent magnets because of the large fields required to overcome anisotropy. We can envision the anisotropy as a field keeping the magnetization parallel to the easy axis. This "field" is called the anisotropy field $\left(\mathrm{H}_{\mathrm{K}}\right)$.

A polycrystalline sample will not exhibit a net magnetocrystalline anisotropy due to the crystal orientations of the different grains. There is another type of anisotropy to be considered: shape anisotropy. Samples prefer to be magnetized along the longer axis. As the axial ratio increases, so does anisotropy. Demagnetizing fields are responsible for shape anisotropy. These are internal fields that created by the magnetization and work against the applied field. The demagnetization field is: $H_{D}=N_{D} M$, where $N_{D}$ is the demagnetization factor which depends on the shape of the sample. $N_{D}$ is small along the long axis and large along the short axis. It would take a larger field to overcome the demagnetizing field of a sample along the short axis than it would for the long one.

Induced anisotropy is also possible by manipulating the directional characteristics of a sample. This type of anisotropy is extrinsic to the material and allows us to engineer the magnetic properties of samples. Magnetic annealing is a common practice, where a sample is heated and cooled in the presence of a magnetic field. This method promotes an easy axis parallel to the applied field. Cold-rolling is a mechanical method that affects grain orientation and shape, also creating directional anisotropy. 


\subsubsection{Magnetostriction}

When a ferromagnetic sample is magnetized it suffers magnetostriction. This effect changes the length of the sample. If it elongates the sample it is said to have positive magnetostriction, and if the sample contracts, negative magnetostriction. These changes affect domains by creating an elastic energy, which is proportional to the volume of the closure domains. Closure domains are those created where flux lines from larger domains close on themselves. To minimize the magnetostriction energy smaller closure domains are formed. Reducing the closure domains would require reducing the other domains causing an increase in exchange and magnetostatic energy! Strong spin-orbit coupling is also linked to large magnetostriction.

\subsubsection{Zeeman Energy}

The Zeeman energy describes the interaction between magnetization of the sample and an applied magnetic field. It describes the energy required to rotate the magnetization of the sample with respect to the applied magnetic field. This value is minimized when the magnetization is pointing in the same direction as the applied field. This energy can be expressed as:

$$
E_{Z}=\int M \cdot H d V
$$

\subsubsection{Domain Walls}

In bulk materials the wall separating ferromagnetic domains are called domain walls or Bloch walls. The magnetization between two domains transitions by 90 or 180 degrees across the wall. The thickness of the wall depends on the competing energy contributions, and wind up being around $10-15 \mathrm{~nm}$ thick. High exchange energies result in wider walls 
because they promote parallel magnetization, so the transition between the domains is much more gradual. Magnetocrystalline energy prefers alignment across the easy axis, therefore walls are thinner due to the sharp transition between domains. Overall, the thickness of the walls is dependent on how the energy is minimized across the sample.

Domain walls also try to minimize the energy of the sample by rotating the magnetization in such a way as to not produce demagnetization. Bloch walls tend to rotate out of the plane of one domain and into the next. In thin films domain walls are known as Néel walls where the spins will rotate in plane from one domain to another. Néel walls are found in thin films because poles will be formed on the film wall rather than the surface, significantly reducing magnetostatic energy.

When an external field is applied, the domain with the magnetic orientation closest to the field direction will grow, reducing the size of the other domains. This growth mechanism is called domain wall motion. When domain walls are set in motion, they can encounter defects in the crystal. These imperfections have magnetostatic energy that keeps the magnetization pinned in a certain direction. A certain amount of energy from an external field is required to overcome the defects. Eventually, the sample is magnetized in a single domain pointing along the easy axis that's closest to the field direction. Further magnetization requires rotating the magnetic dipole moments away from the easy axis towards the direction of the field. The larger the magnetocrystalline anisotropy, the larger the field required to saturate the sample. The removal of the field will cause the magnetic dipole moments to rotate back to the easy axis. With the field gone, demagnetization forces will begin to kick in developing domains once again. These demagnetization forces usually aren't strong enough to overcome energy barriers from the defects. This 
results in hysteresis, where the extra needed field to bring the sample back to zero magnetization is called the coercive field. It is apparent that samples with more defects tend to be harder than high-purity ones.

\subsection{Nanoparticles and Thin Films}

\subsubsection{Nanoparticles}

When we scale below a critical size, magnetic particles may only have a single domain. If domain walls are typically $\sim 100 \mathrm{~nm}$, nanoparticles below this size can only exist as a single domain. A single domain particle will have high magnetostatic energy due to the absence of other domains to account for the stray fields. There is also a clear relation between magnetostatic energy and the volume of the particle. Single domain particles always remain saturated and those with more than one domain can only be kept saturated with a field larger than the demagnetizing field.

To magnetize a single domain particle, only the anisotropy must be overcome. Magnetization of a single domain particle lies on the easy axis, determined by both magnetocrystalline anisotropy and shape anisotropy. To switch magnetization in the opposite direction, the magnetic dipole moments must rotate through the hard axis and on to the easy axis along the applied field. Particles with large anisotropy will have large coercivity, making them ideal for magnetic media [22]. Typical hysteresis loops for single domain particles along the easy axis are square since they have well defined switching fields. In the hard direction there is no hysteresis since the field just rotates the spins out of the easy direction and the instant that it's removed, the magnetization is gone. Nanoparticles are exploited for their square hysteresis, so they must be appropriately aligned to exploit the easy axis. 
Single domain particles can have high coercivity, but if they are scaled down below a certain size they can become superparamagnetic. At this size, the coercivity is zero because of the reduced anisotropy with size. The total anisotropy energy is: $K V$, where $\boldsymbol{K}$ is the anisotropy constant and $\boldsymbol{V}$ is the volume of the nanoparticle. Should $K V$ become comparable to $k T$, the thermal energy could randomly switch the magnetization from one easy axis to the other in the absence of an applied field. When superparamagnetic, magnetization is closer to that of a paramagnetic material with a large magnetic moment. The time between flips is known as the Néel relaxation time $\left(\boldsymbol{t}_{N}\right)$. It relates to both the thermal energy and the anisotropy energy and is expressed as:

$$
t_{N}=t_{0} e^{\left(\frac{K V}{k_{B} T}\right)}
$$

The Néel relaxation time $t_{N}$ also depends on $\boldsymbol{t}_{\boldsymbol{0}}$ which is a property of the material called the attempt time. If the time to measure magnetization is larger than the Néel relaxation time, the net magnetization will be zero, rendering it superparamagnetic. High anisotropy is necessary if we want to use small nanoparticles for magnetic media applications. In fact, for volatile memory applications the thermal stability ratio $\left(K V / k_{B} T\right)$ must greater than 20 .

Aggregation of particles can also affect the coercivity [23]. Should the anisotropy be primarily due to shape, we find that as the particles get packed close together the lower the anisotropy will be. This is primarily due to particle interaction, where the field of a nanoparticle can magnetize neighboring nanoparticles. Isolated particles will have a higher coercivity than a cluster of particles. 


\subsubsection{Thin Films}

Magnetic thin films are currently most relevant technologically. Thin films are made by depositing atoms onto a substrate. This can be done by physical vapor deposition techniques such as thermal evaporation and sputtering, or chemical vapor deposition techniques like atomic layer deposition (ALD). Of the many deposition techniques, sputtering is the most popular in magnetic media because it allows the user to deposit almost any number of materials with reproducible results [24].

Thin films exhibit unique magnetic behaviors that wouldn't be found in bulk since the number of atoms at the surface become comparable to the number found in the volume. Sputtered films tend to have small grain sizes $(\sim 100 \mathrm{~nm})$ making them comparable in size to the domain wall thickness. Several grains can be part of a domain wall, where each grain can have different crystallography. Texture can therefore play a key role in the magnetocrystalline anisotropy. It is important to monitor the deposition conditions to avoid any unwanted texture.

Typically, magnetization is found in the plane of the film. Shape anisotropy is mostly responsible since large demagnetizing forces are present out of plane due to the aspect ratio between the film thickness and the surface area. However, surface anisotropy can also occur where the magnetic dipole moments align perpendicular to the surface of the

film. Thin magnetic films can have a change in the magnetocrystalline anisotropy from the lack of neighboring atoms at the surface. The competing surface and shape anisotropies are found to be a function of thickness, where really thin films $(<2 \mathrm{~nm})$ prefer perpendicular orientations. For example, a film a $\mathrm{CoFeB}$ that is less than a couple nanometers will become magnetized out-of-plane rather than in-plane. In thin films, 
domains are typically separated by Néel walls rather than Bloch walls to reduce magnetostatic energy.

Given the greater amount of surface to volume ratio, interfaces between different films and substrates can also produce interesting behavior. Magnetization can therefore be dramatically different at the surface of a material than at the bulk. For example, for materials and substrates with similar lattice constants, the deposited material will tend to match its in-plane constant to that of the substrate. This effect can cause strain on the film which can affect its magnetic behavior. Changing the local bond lengths and coordination environment can alter spin states substantially significantly changing magnetic properties. Changes in strain also affect the magnet via magnetostriction. Depositing materials with near atomic precision allows us to engineer alloys with many different layered configurations, which can have an impact on the anisotropies. Even interfacing with nonmagnetic materials can generate a whole new class of physics. Giantmagnetoresistance (GMR) is a consequence of interfacing magnetic with nonmagnetic thin films [25], and is an important Nobel prize winning effect that propelled magnetics into the magnetic storage industry.

\subsection{Magnetoresistance}

When a magnetic field is applied to a material it can alter its electrical resistance. This change in resistance is called the magnetoresistive ratio which takes the difference of

resistance divided by the resistance when the field is zero $\left(\frac{R_{H-} R_{0}}{R_{0}}\right)$. Materials with increased electrical resistance when a field is applied have positive magnetoresistance, and those with reduced resistance negative magnetoresistance. There are many 
technological applications that exploit magnetoresistance, especially in magnetic media. This subsection is focused on chronologically going through the important types of magnetoresistance and their impact in magnetoelectric devices. The most important type of magnetoelectric devices relevant to this thesis are those composed of multilayered structures, particularly those made of two ferromagnetic layers separated by a nonmagnetic spacer.

\subsubsection{Anisotropicmagnetoresistance}

During conduction, electrons scatter but will travel in a relatively straight path. When we apply a magnetic field Lorentz forces will curl the electrons, scattering them even further. This effect is not appreciable in most metals, however in ferromagnets it is much more pronounced. Anisotropicmagnetoresistance in ferromagnets have been reported to be as high as $5 \%$ at room temperature. The direction of the magnetic field also impacts the resistance, making this effect anisotropic. The resistance of the ferromagnet when current flows parallel to an applied magnetic field increases with field strength. When current flows in the direction perpendicular to the magnetic field it decreases in resistance by an equal amount.

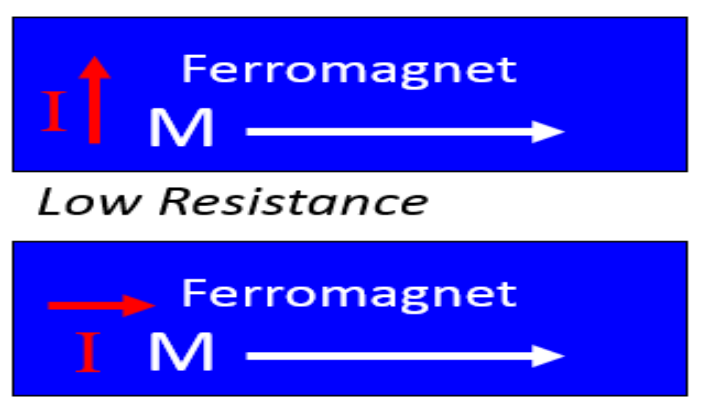

High Resistance

Figure 2-12: AMR Low and High Resistance 
Anisotropicmagnetoresistance (AMR) is attributed to spin-orbit coupling [26]. The conduction of electrons in the s-band are scattered by the unquenched part of the orbital angular momentum of the $3 \mathrm{~d}$ electrons. The direction in which the electron could deviate when a magnetic field is applied impacts the level scattering. When the field is in the same direction as the current, the electron orbits are perpendicular to the current, increasing the chance of scattering. A perpendicular field will rotate the orbits parallel to the current, reducing the scattering cross-section. There is also a temperature dependence in both magnetic and nonmagnetic materials. With increased thermal agitations, there will be more scattering. In ferromagnets, below the Curie temperature, a decrease in temperature also means an increase in the order of magnetic moments. This enhanced magnetic order results in an improved magnetoresistance since scattering is further diminished. The magnetoresistance can be calculated as:

$$
A M R=\frac{R_{\|}-R_{\perp}}{\frac{1}{3} R_{\|}+\frac{2}{\cdot} R_{\perp}}
$$

\subsubsection{Giantmagnetoresistance}

Changes of resistance can be enhanced further by utilizing multilayers of thin films, where ferromagnetic films are separated by non-magnetic conductors [25]. This metallic multilayer arrangement creates a quantum mechanical magnetoresistive effect coined Giantmagnetoresistance (GMR). The electrical resistance depends on the relative magnetization orientation of each ferromagnetic layer. The magnetization of each layer is can be manipulated by an applied external magnetic field. Albert Fert and Peter Grünberg were awarded the Nobel Prize in physics for the discovery of GMR. 
The performance of GMR depends on the interlayer exchange coupling and the spin dependent transport [27]. The interlayer exchange coupling is responsible for the magnetization orientation of the ferromagnetic films. The thickness of the non-magnetic spacer layer determines if the magnetic layers couple ferromagnetically or antiferromagnetically. An area of focus in GMR is understanding the different coupling mechanisms. RKKY is an example of a coupling mechanism that exhibits an oscillatory behavior in magnetic orientation as the thickness of the spacer layer changes. Initially, for thin spacers, ferromagnetic coupling is preferred. As the spacer thickness is increased, an antiferromagnetic coupling prevails. Further increasing the thickness brings the magnet coupling back to ferromagnetic, and so on and so forth. As the thickness increases, the strength of the coupling decreases.

Without an applied field, the multilayer stack is set up in an anti-parallel fashion, meaning the interlayer exchange coupling is negative. When a field is applied to break the interlayer coupling, the magnetic orientations favor a parallel alignment. GMR is measured as the difference between the antiparallel configuration and the parallel configuration. When the films are antiparallel, spins with orientations opposite of the regions of magnetization will strongly scatter increasing the resistance. Spins with the same orientation will scatter much less, decreasing the resistance. 

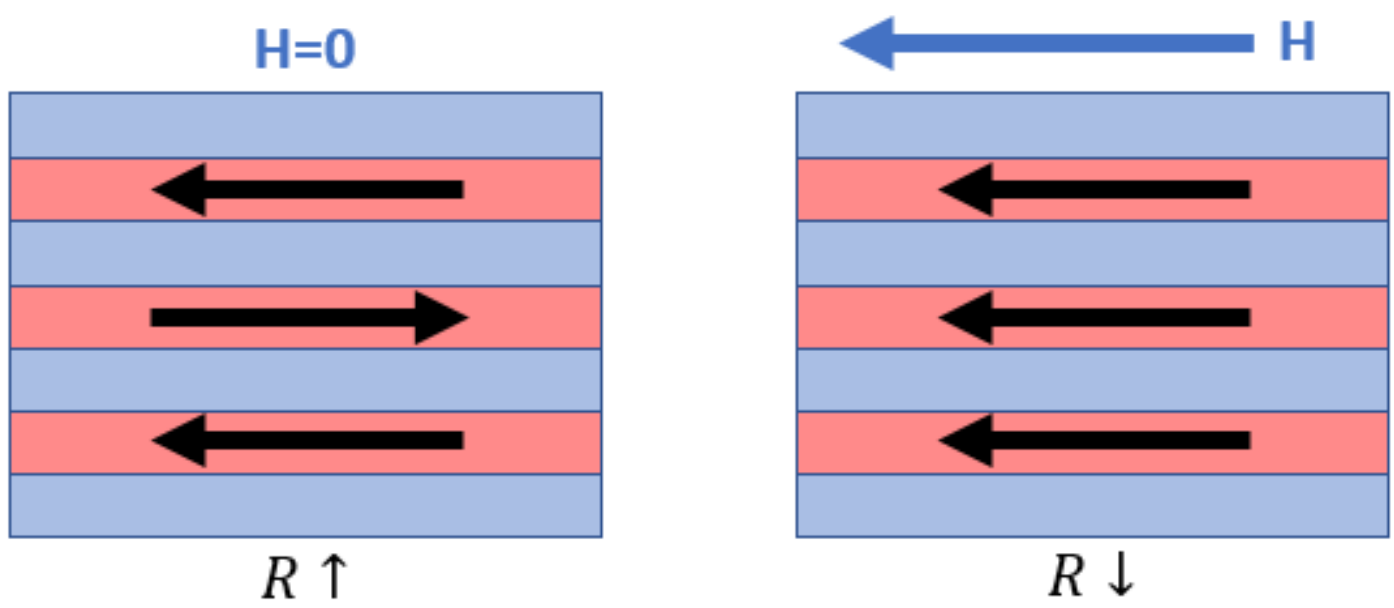

Figure 2-13: High and Low Resistance in GMR Junctions

This scattering effect is understood with band theory. Nonmagnetic metals have an equal amount of spin-up and spin-down electrons at the Fermi level. Electrons of either spin direction can travel through the conductor with equal probability. In ferromagnets, however, there are more available states at the Fermi level for one spin direction than the other. These materials are then considered half metals, since only electrons with spins of the same orientation as the spin state at the fermi level can conduct. In an antiferromagnetic configuration, an unpolarized current will flow through one layer allowing only the electrons with the spin orientation of the magnetic film. The current becomes polarized with one spin orientation, but will be met with resistance as it tries to flow through the following film that has an opposite magnetic orientation. If all the magnetic films have the same orientation, the spin polarized current will flow through the multilayer structure unimpeded. Magnetoresistance can be measured as:

$$
G M R=\frac{R_{\max }-R_{\min }}{R_{\min }}=\frac{R(H=0)-R(H \neq 0)}{R(H \neq 0)}
$$


Antiparallel Magnetic Films:
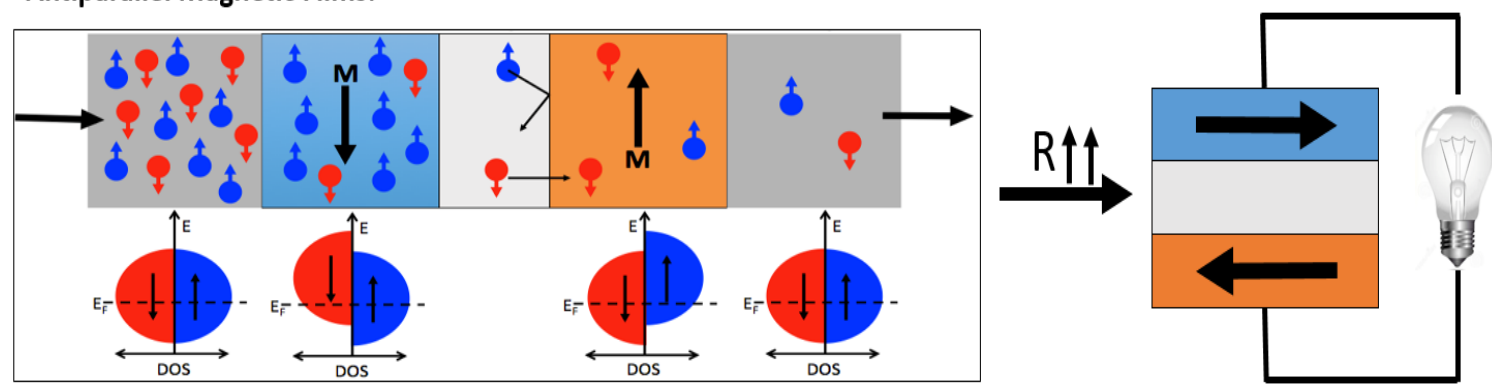

Parallel Magnetic Films:
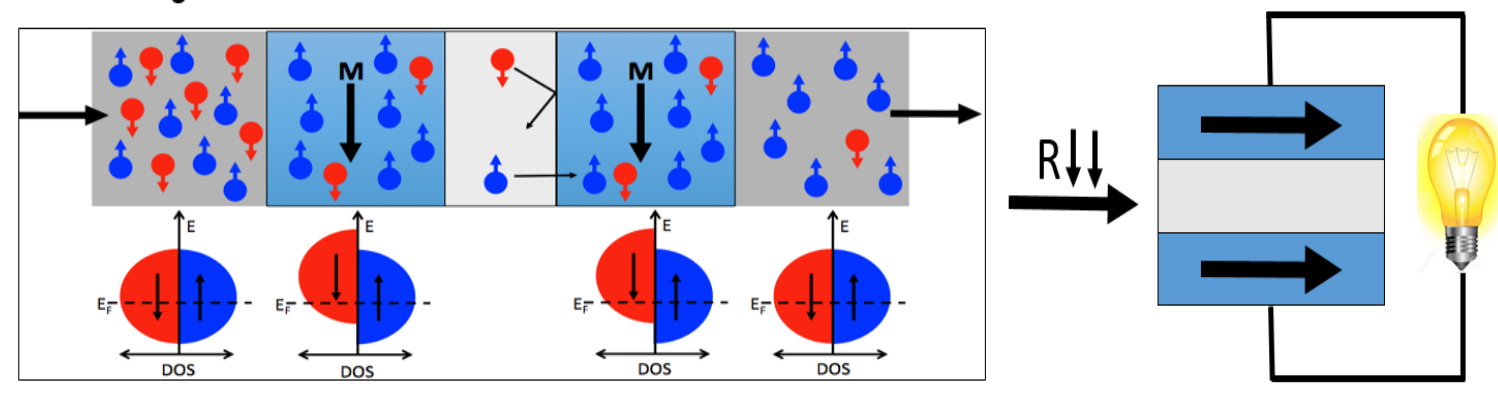

Figure 2-14: Parallel VS Antiparallel Configuration

Spin valves are a type of multilayer stack that consists of four films: Two ferromagnetic films, one non-magnetic spacer film, and one antiferromagnetic film. The antiferromagnetic film is used to "pin" one of the ferromagnetic layers via exchange interlayer coupling. Interfacing an antiferromagnet with a ferromagnet results in large negative exchange coupling energy. This bilayer interface generates an increase in coercivity of the ferromagnet, creating exchange anisotropy. Quantifying exchange anisotropy is an on-going research challenge. While the pinned ferromagnetic layer has a fixed magnetization with large coercivity, the other ferromagnetic layer is magnetically soft and free to switch back and forth. By manipulating the free layer, both antiparallel and parallel configurations are possible.

The magnetoresistance achieved in GMR has been as high as 65\% at room temperature, an order of magnitude greater than AMR. GMR has had an enormous impact in magnetic recording, replacing AMR heads in hard disk drives [28]. Bits can be defined by the 
relative orientations of the multilayer: Parallel (1) and antiparallel (0). These bits are distinguished because of the different resistance of each magnetization configuration. Using magnetics to store data has a major advantage: data is retained without needing power to maintain its magnetization, reducing the overall power consumption required to hold data. This form of memory is called non-volatile. Semiconductor based memory is volatile, requiring power to maintain their memory.

\subsubsection{Tunnelingmagnetoresistance}

GMR eventually evolved into tunnelingmagnetoresistance (TMR) by replacing the nonmagnetic metallic spacer layer that separates the two ferromagnetic layers with an insulating layer instead. This insulating layer is very thin $(\sim 1 \mathrm{~nm})$ and is called the tunnel barrier. Classically, electrons cannot conduct through an insulating barrier, but quantum

mechanics allow it. Tunneling occurs due to the wave nature of electrons. An electron approaches a barrier where the electron wave inside the barrier becomes evanescent, decreasing exponentially in amplitude as it travels thru. The barrier must be thin enough so as not to completely diminish the electron wave all together. There is an exponential dependence between the tunnel barrier thickness and the conduction of the current travelling through it. The tunneling probability is proportional to the ratio between the transmitted and incident electron waves. As expected, if the ferromagnetic layers have the same magnetic orientation the resistance is low, when they are anti-parallel, it's high. Jullière discovered the TMR effect in 1975 when he noticed the change of resistance of around $\sim 14 \%$ in a $\mathrm{Fe} / \mathrm{Ge}-\mathrm{O} / \mathrm{Fe}$ junction at a temperature of $4.2 \mathrm{~K}$ [29]. Other insulators, such as aluminum oxide and magnesium oxide have shown significantly higher 
magnetoresistance at room temperature [30,31,32]. Devices composed of two ferromagnets separated by an insulator are called magnetic tunnel junctions (MTJ).
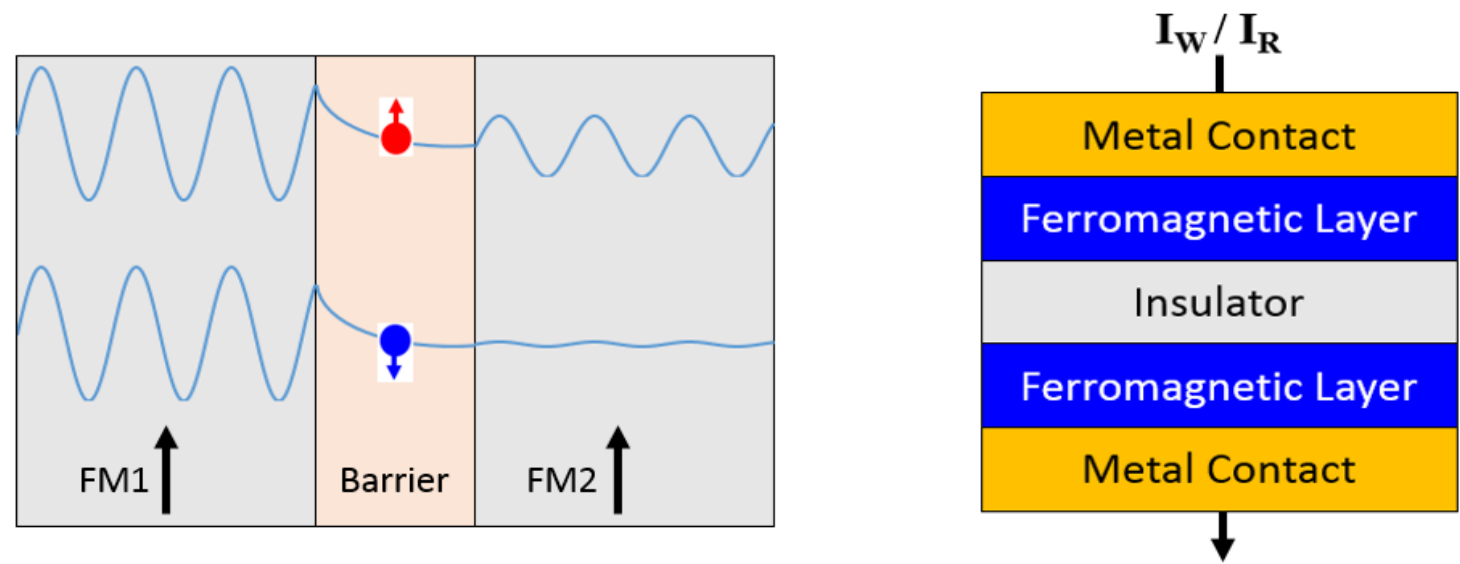

Figure 2-15: Spins tunneling through barrier (a); MTJ Structure (b)

The Jullière model assumes preservation of spin orientation across the tunnel barrier so electrons can only tunnel into a band with the same spin orientation [33]. The tunneling current therefore depends on the product of the density of states (DOS) of both ferromagnetic layers. When a voltage is applied, the energy level shifts and current conducts through the barrier. When the magnetic layers are magnetized in the same direction, the electrons at the Fermi level of the first magnetic layer will tunnel to the second. The electrons with one spin direction will occupy the states available for that spin direction, and the same is true for the other electrons with opposite spin. The electrons will effectively go from majority band to majority band, and minority band to minority band: $G_{P}=D_{1}(\uparrow) D_{2}(\uparrow)+D_{1}(\downarrow) D_{2}(\downarrow)$, where $\mathrm{D}$ represents the DOS, specifying the ferromagnetic layer and the magnetic orientation. When the magnetic moments of the ferromagnetic thin films are anti-parallel, the electrons conduct from the minority band to the majority band and vice versa: $G_{A P}=D_{1}(\uparrow) D_{2}(\downarrow)+D_{1}(\downarrow) D_{2}(\uparrow)$. For high tunneling conduction, the density of states for both majority and minority bands of both 
ferromagnetic layers must be the same so $G_{\mathrm{P}}>>\mathrm{G}_{\mathrm{AP}}$. The tunneling spin polarization can be calculated from the spin dependent density of states at the Fermi level:

$$
P=\frac{D_{1}(\uparrow)-D_{2}(\downarrow)}{D_{1}(\uparrow)+D_{2}(\downarrow)}
$$

The magnetoresistance is expressed as:

$$
T M R=\frac{R_{A P}-R_{P}}{R_{P}}
$$

Resistance is the reciprocal of conductance $\left(R=\frac{1}{G}\right)$. The TMR ratio can also be expressed using Jullière's formula:

$$
T M R=\frac{2 P_{1} P_{2}}{1-P_{1} P_{2}}
$$

The parameters $p_{1}$ and $p_{2}$ are the spin polarization factors for each ferromagnetic layer. Maximum TMR $(\infty)$ can be achieved when the polarization equals 1 for both ferromagnetic layers.

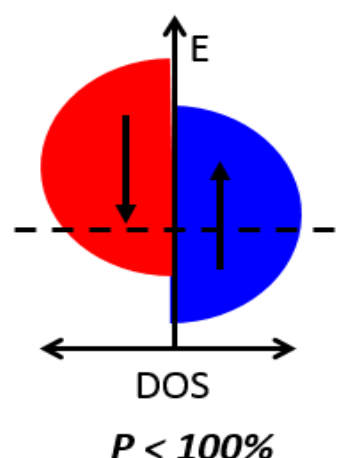

$T M R<\infty$

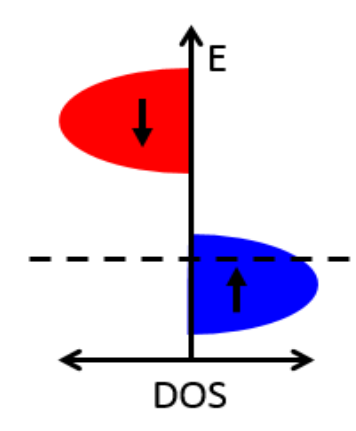

$P=100 \%$

$T M R=\infty$

Figure 2-16: Different levels of polarization in a ferromagnet

TMR values have superseded GMR by an order of magnitude, with the highest recorded being $\sim 600 \%$ at room temperature [34]. While theoretically high TMR is possible by finding ferromagnets with high polarization factors, Jullière's model does not account for surface quality of the films. For example, grain boundaries in the films or defects in the 
tunneling barrier can significantly impact the TMR ratio. Interface and insulator properties are also excluded in Jullière's assumptions. Optimizing the film quality and understanding the interfaces in tunneling devices is critical for obtaining high TMR. MTJs in this thesis are configured to have TMR and they will be discussed in more detail in chapter 4 .

\subsection{Spin Transfer Torque}

Electric current is usually unpolarized, where $50 \%$ of electrons have an upward spin orientation and the other $50 \%$ have a downward one. When an unpolarized current passes through a magnetic material, it can be polarized in the same direction as the magnetic material. This is because electrons with the same spin orientation as the magnetization of the material scatter less than those with the opposite orientation. However, when a spin polarized current conducts through a magnetic material with opposite magnetization, the spins from the electrons can transfer spin angular momentum, creating a torque between the electrons and the magnet. This transfer of momentum can generate oscillations in the magnetic layer and even switch its magnetic orientation all together. The advantage of STT is the ability to manipulate magnetization electrically rather than with external magnetic fields. Slonczewski and Berger independently theorized this phenomenon in $1996[13,35]$.

Magnetic tunneling junctions have two ferromagnetic layers separated by an insulting layer. One of the ferromagnetic layers is magnetically hard or "pinned" and the other is soft or "free." Typically, a current is polarized from the pinned layer and utilizes STT to switch the free layer from an antiparallel alignment to a parallel one. When an electron flows through a ferromagnetic layer with a magnetic moment that isn't collinear with the 
magnetic moment of the electron, the magnetization of the ferromagnet will exert a torque on the spin of the electron to align it. Due to conservation of angular momentum, the reverse also occurs where the electron's spin will exert a torque on the magnetization of the ferromagnet. This effect is only observed in nanoscale magnetic structures, typically less than $200 \mathrm{~nm}[36,37,38]$. Should we want to go from an antiparallel to parallel configuration in the $\mathrm{MTJ}(\mathrm{AP} \rightarrow \mathrm{P})$, we flow positive current where electrons conduct from the fixed layer to the free layer. For a parallel to antiparallel configuration $(\mathrm{P} \rightarrow \mathrm{AP})$, we flow a negative current where electrons conduct from the free layer to the fixed one instead.
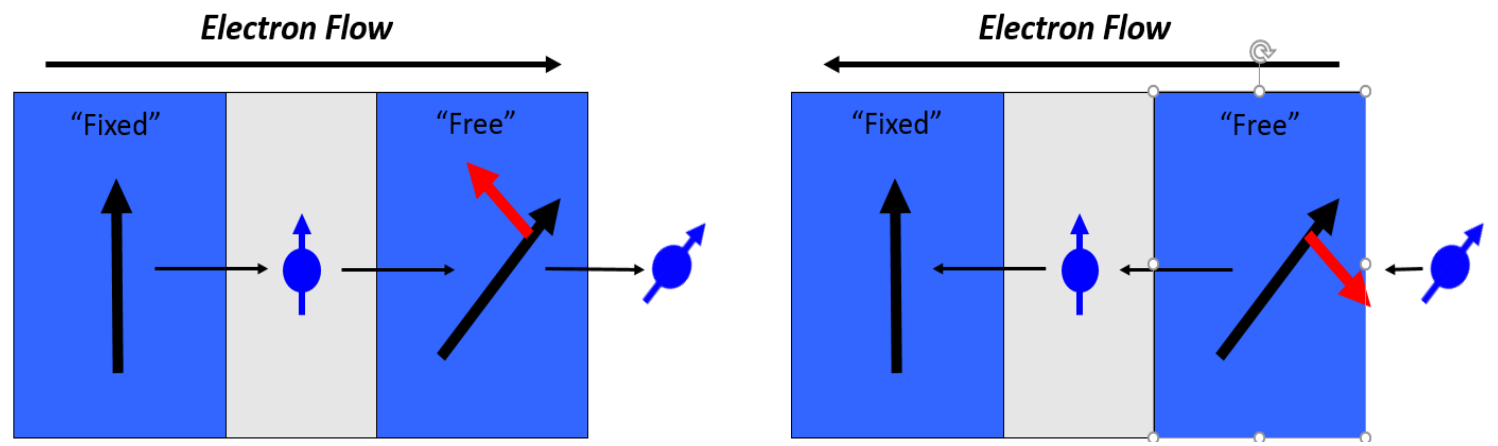

Figure 2-17: Spin transfer torque in both directions

The change in magnetization in the ferromagnetic layer due to STT can be described by the Landau-Lifshitz-Gilbert (LLG) equation [39]:

$$
\frac{d M_{\text {free }}}{d t}=\gamma\left(M_{\text {free }} \times H_{\text {eff }}\right)-\alpha\left(M_{\text {free }} \times \frac{d M}{d t}\right)+\beta J\left(M_{\text {free }} \times M_{\text {free }} \times M_{\text {fixed }}\right)
$$

$\boldsymbol{M}_{\text {free }}$ is the magnetization of the free layer, $\boldsymbol{M}_{\text {fixed }}$ is the magnetization of the fixed layer, $\boldsymbol{\gamma}$ is the gyromagnetic ratio, $\boldsymbol{H}_{\text {eff }}$ is the effective field, $\boldsymbol{\alpha}$ is the damping coefficient, $\boldsymbol{\beta}$ is an STT coefficient, $\boldsymbol{J}$ is the current density. The first term describes the precession of the magnetization about the effective field. This field is the sum of the externally applied magnetic field, anisotropy field, exchange field, demagnetizing field, etc. The second is a 
damping term that describes the energy loss to the magnetic system, gradually decreasing the precessional angle. The final term is Slonczewski's, and it considers the STT effect. Switching magnetizations depends on the competing behavior of STT term $(\boldsymbol{\beta})$ and damping coefficient $(\boldsymbol{\alpha})$. If the STT component and the current density are small compared to the damping coefficient, the magnetic dynamics dampen until a state of equilibrium is reached. Increasing the STT component beyond the damping coefficient can excite the magnetization to precess at larger angles. With sufficient current density, the spin torque can dominate the magnetic dynamics and switch the magnetization direction of the ferromagnet altogether [40,41]. Switching magnet states is heavily influenced by the direction and magnitude of the current density. Magnetic states can therefore be manipulated with a local injection of spin polarized current!

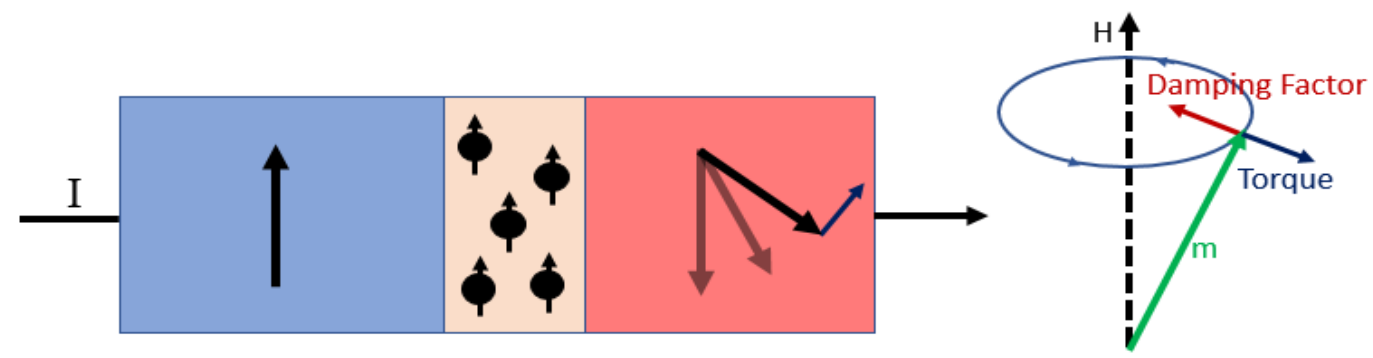

Figure 2-18: Spin Transfer Torque Illustration

A critical current $\left(I_{C}\right)$ is defined as the minimum current required to switch the magnetization at $0 \mathrm{~K}$. Switching involves a precessional motion of the magnetization which goes increasing in magnitude. When the precessions are large enough that they reverse the direction of magnetization, it quickly dampens and precesses with a small amplitude in the switched direction. This precession circulates in the order of $\mathrm{GHz}$, where switching can occur in the order of nanoseconds. Switching time when using a spin polarized current is directly proportional to the amount of current $\left(t_{S W} \sim \frac{1}{I-I_{C}}\right)$. Currents 
smaller than $I_{C}$ generate small precession, but not enough to switch. However, thermal excitations can assist in the switching process, allowing currents smaller than $I_{C}$. However, thermally assisted switching techniques complicate the architecture of the overall device. Switching via spin transfer torque has already made its way into the commercial sector in the form of STT-MRAM devices. Research in this field is focused in reducing the current required to switch magnetic states. 


\section{EXPERIMENTAL METHODS}

Micro and nanofabrication combine an array of technologies with the expectation of making high quality devices in the micrometer and nanometer range. Devices generally begin with a proper choice in substrate and can go through many processes such as thin film deposition, lithography, etching, etc. These processes have proven fundamental in many technological applications such as integrated circuits, microelectromechanical system (MEMS) and of course, magnetic tunnel junctions (MTJ). Most micro and nanofabrication is done in a cleanroom environment where the number of airborne particles are controlled. Cleanrooms are categorized according to the number of particles of a certain dimension per unit volume. This chapter focuses on some of the most important processes used in developing the MTJs in this thesis. The following 4 books have helped me develop my skills in fabrication in the cleanroom and have proven indispensable: Fundamentals of Microfabrication [42] by Marc J. Madou, Principles of Plasma Discharges and Materials Processing [43] by Michael A. Lieberman and Allan J. Lichtenberg, Handbook of Thin Film Deposition [44] by Krishna Seshan and A User's Guide to Vacuum Technology [45] by John F. O'Hanlon. They have been an excellent reference when planning the extensive processing required in this thesis. Most of the information in this section is based on the books mentioned.

\subsection{Substrate Choice and Preparation}

Micro- and Nano-electronic devices are for the most part not "free-standing," but developed on top of a support substance called a substrate. In electronics, substrates are either semiconductors or electrical insulators. Substrates are thin slices of solid, planar material also called wafers. Common material choices for wafers include: silicon, silicon 
dioxide, aluminum oxide, sapphire, germanium, gallium arsenide, etc. Devices developed on a given substrate undergo micro and nanofabrication processing such as photolithography, etching, film deposition, dicing and so forth. Substrates, in addition to material properties that can contribute to the devices performance and testing, serve primarily as a handle for each fabrication step. On a single wafer many devices can be arrayed and processed in parallel, and towards the end of the fabrication process they are diced and packaged individually.

Choosing an appropriate substrate is an important step in ensuring high quality devices. The substrate must be compatible with the fabrication process and test bed. In our case, the substrate must be a good insulator, flat, have good metal oxide adhesion, high thermal conductivity, and mechanical stability. The best candidate for the devices developed in this thesis was silicon with thermally grown silicon dioxide. This choice was also made because fabrication processes that involve silicon substrates are well known and studied, significantly reducing the amount of time needed to characterize many processing steps and checking for compatibility. Additionally, the ease of which these wafers can be handled (mechanical robustness), cost, and obtainability was a factor in this decision. The exact substrate is a $100 \mathrm{~mm} \mathrm{Si}$ wafer that is $500 \mathrm{um}$ thick, with a $300 \mathrm{~nm}$ thermally grown $\mathrm{SiO}_{2}$. The size and thickness of the substrate was chosen for equipment and processing compatibility.

Before beginning the fabrication process, the substrate must be properly prepared and cleaned. Cleaning minimizes the presence of any unwanted particles, grease, and free metal ions that inhibit the performance of the MTJs. The samples were rinsed in various solvent baths and sonicated in each step before drying. Each wafer would be submerged 
in an acetone bath and sonicated for 20 minutes, followed by sonication in Isopropanol for $20 \mathrm{~min}$ and rinsed thoroughly with DI-Water. Solvents clean oil and organic residue on the surface of the wafer. Solvents, especially acetone, leave their own residues, which is why we use isopropanol and DI-water to rinse afterwards. Wafers are then placed in a Spin Rinse Dryer (SRD) that provides a uniform DI-water clean and rinse cycle followed by a heated $\mathrm{N}_{2}$ drying step. The wafers are then checked for any unwanted residue, grease marks and scratches under an optical microscope. This method is effective for ensuring clean substrates, paramount for high quality devices and acceptable yield.

\subsection{Thin Film Deposition}

A thin film is a layer a material that can be as thick as a few atoms to several hundred micrometers. This film is generally applied onto a substrate or on a previously deposited

coating. Thin films play a critical role in the manufacturing process for a plethora of applications, such as: semiconductor devices, MEMS, magnetic recording media, solar cells, optical devices, etc. Thin film deposition is an important process in device development. Different techniques are employed for depositing these films, each with its advantages and disadvantages. This section will be dedicated to briefly covering different thin film deposition techniques and focusing in more detail on those used for the fabrication of the devices developed in this thesis.

Thin film deposition can be classified into two very broad categories: Chemical Vapor Deposition (CVD) and Physical Vapor Deposition (PVD). Chemical deposition involves one or more volatile vapor-phase precursors that undergo a chemical change at the surface of a substrate, leaving a deposited coating. The reactions can be assisted by heat (Thermal CVD), high frequency radiation (photo-assisted CVD) or plasma (Plasma- 
Enhanced CVD). If any by-products are produced, they are removed via gas flow. CVD tend to produce high quality solid films and is very common method used in the semiconductor industry. In fact, Atomic Layer Deposition (ALD), a newer form of a CVD process, has the capability of providing a very controlled method for depositing ultra-thin layers with atomic level precision. Despite that only a certain number of pure elements can be deposited with this technique, it is more common to deposit compounds. Popular materials include: Dielectric Oxides $\left(\mathrm{SiO}_{2}, \mathrm{TiO}_{2}\right.$, etc. $)$, Elemental Semiconductors ( $\mathrm{Si}$, Ge, etc.), III-V Compounds (GaAs, InP, AlGaAs, etc.), Metal Nitrides $\left(\mathrm{Si}_{3} \mathrm{~N}_{4}, \mathrm{TaN}\right.$, etc.), Metal Carbides ( $\mathrm{SiC}, \mathrm{TiC}$, etc.), Metals (Ta, W, etc.), etc. The main advantage of CVD is that it's conformal because it reacts with any exposed surface. This allows for uniform film thickness on the sidewalls and undersides of features, even those with high aspect ratio. CVD also has several disadvantages including the elevated temperatures required to deposit films, and the precursors required for deposition need to be volatile near room temperature and tend to be highly toxic, explosive or corrosive. PECVD is a deposition method of choice for depositing $\mathrm{SiO}_{2}$ and $\mathrm{Si}_{3} \mathrm{~N}_{4}$ as electrical insulation and passivation for some of the devices made in this thesis.

Physical vapor deposition involves a physical release from a source material onto a substrate via mechanical, thermos-dynamical or electro-mechanical processes. Atoms or molecules are vaporized from a solid or liquid source and transferred and condensed onto a substrate via a vacuum or low pressure gaseous environment. PVD processes can deposit many kinds of films including elements, alloys, compound materials, and some polymers. Common PVD processes include: Thermal Evaporation, a deposition method that relies on resistively heating the material; Electron Beam Evaporation, similar to 
thermal evaporation but the material is vaporized by electron beam bombardment; Molecular Beam Epitaxy, a technique for epitaxial growth by means of interaction of one or more molecular or atomic beams on the surface of a heated crystalline substrate; Cathodic Arc Deposition, where a high power electric arc vaporizes a cathode target; and Sputtering where material is ejected from a target from collisions of incident energetic particles. Electron beam evaporation and sputtering are the two main PVD film deposition methods used to fabricate devices in this thesis and will be discussed in more detail later in this chapter. PVD has the advantage of not requiring high temperatures to deposit materials, or hazardous precursors and byproducts. Unlike CVD, most PVD coating techniques are line-of-sight, so step coverage becomes a challenge.

Depositing thin films is not always a straightforward process. When developing magnetic tunneling junctions the properties of the films, such as grain size and texture, play an important role in the overall performance of the devices. Process parameters (process pressure, base pressure, gas flow, substrate heating, etc.) must be carefully characterized to obtain high quality films. Film characterization was done on bare silicon chips. Atomic Force Microscopy (AFM), a scanning probe technique, was used to determine the surface topology of the deposited films where RMS roughness values can be extrapolated. AFM makes use of a sharp tip on a cantilever that scans over the surface of the film and interacts with the atomic potentials of the surface atoms. Transmission Electron Microscope (TEM) images were also used as a visual aid for determining film roughness. The roughness of the electric contacts where the tunnel junction will be deposited on could drastically alter the overall performance of the device. The rougher the electrode, the less uniformity the tunnel junction will have, especially the tunneling film. Should the 
film be too rough, hot spots can be created across the junction. These spots are thin barrier regions with a lower resistive path. Due to the exponential relation of the conductance with the oxide thickness, large amount of current can flow with a low voltage bias in these hot spots. These large, localized currents can lead to heating that could break down the tunnel barrier which in turn can lead to electrical shorts. Hot spots will dominate the electron transport, deteriorating the spin dynamics and the overall TMR. Roughness at the interface between the ferromagnetic layers can also result in “orange peel" coupling [46]. This orange peel coupling (also known as Neel Coupling) occurs between the two magnetic layers from the MTJ creating magneto-static interactions from local magnetic poles at the rough interface. If the surface of the magnetic material were flat, then the magnetization would follow the surface profile. A rough interface generates a strong intralayer exchange creating local magnetic poles across the surface of the magnetic films. The ferromagnetic coupling energy $\mathbf{J}$ can be described with the following equation:

$$
J=\frac{\pi^{2} h^{2}}{\sqrt{2} \lambda}\left(\mu_{0} M M^{\prime}\right) e^{\left(\frac{-2 \pi t_{b} \sqrt{2}}{\lambda}\right)}
$$

The constant $\boldsymbol{\mu}_{\boldsymbol{0}}$ is the permeability of free space, $\boldsymbol{M}$ and $\boldsymbol{M}$, are the saturation magnetization of the ferromagnetic films, and $\boldsymbol{t}_{\boldsymbol{b}}$ is the thickness of the barrier. The interface roughness is quantified by the amplitude $\boldsymbol{h}$ and the wavelength $\lambda$. The image below visually represents these roughness parameters, where the interface roughness is approximated as a 2-dimensional sinusoid. The coupling field $\boldsymbol{H}_{N}$ can be expressed as: $H_{N}=\frac{J}{\mu_{0} M t_{f}}$, where $t_{f}$ is the thickness of the ferromagnetic layer. It is quite clear that the rougher the surface, especially if the amplitude of the roughness $(\boldsymbol{h})$ is large, the larger 
the coupling energy and field. Strong coupling may cause both ferromagnetic layers to switch together, resulting in no change in resistance.

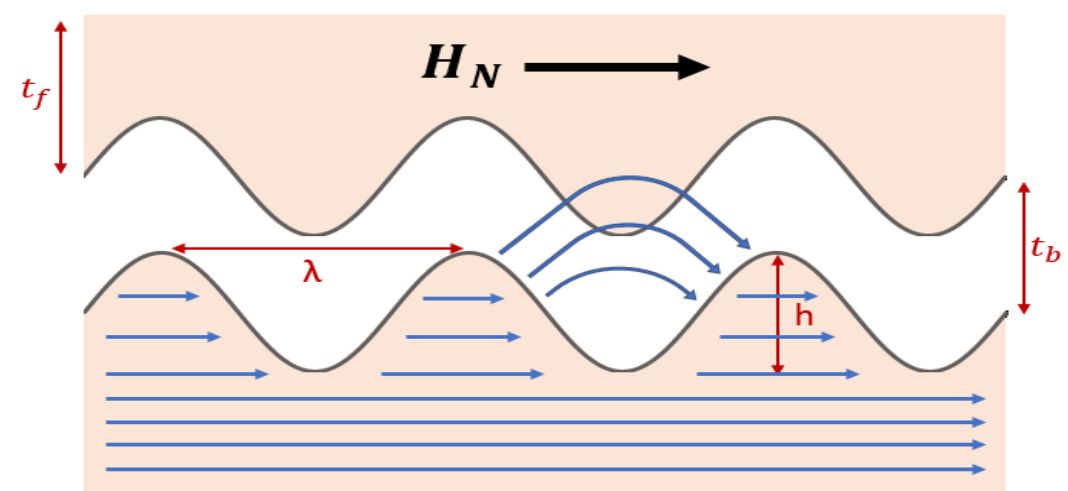

Figure 3-1: Orange Peel Coupling Due to Surface Roughness

Texture also plays a key role in obtaining high TMR in magnetic tunnel junctions. Analyzing the texture involves evaluating the distribution of crystallographic orientations of the grains in a polycrystalline film. For example, to produce high TMR, the tunnel barrier $\mathrm{MgO}$ must have [001] texture and it must be lattice matched between the [001] plane of body-centered-cubic (bcc) CoFeB films. X-ray diffraction (XRD) is a nondestructive method for determining crystal structure. The reason x-rays are used is because their wavelength is within the same order of magnitude as the spacing between crystal planes. An x-ray diffraction (XRD) tool was used to determine the crystallinity in films indicating both the crystalline order and texture relative to the normal direction.

\subsubsection{Sputtering}

Sputtering is a physical vapor deposition technique where a target material is bombarded with positively charged gas ions (ex: Argon) with enough momentum transfer to overcome the surface binding energy allowing the atoms of the target material to be ejected. A substrate is placed in a vacuum chamber containing an inert gas (such as Argon) and a voltage is applied between the target material and the substrate. The target 
acts as the cathode and the substrate as the anode. Free electrons flow from the cathode (target) and collide with the outer shell of the sputtering gas atoms, driving their outer electrons off $\left(\boldsymbol{e}^{-}+\boldsymbol{A r} \rightarrow \mathbf{2} \boldsymbol{e}^{-}+\boldsymbol{A} \boldsymbol{r}^{+}\right)$. Each collision results in an extra electron being knocked off which in turn can collide with other gas atoms, creating a cascade effect known as impact ionization. Due to the ionization of the sputtering gas, the positively charged gas ions bombard the target at very high velocities, releasing source material. Plasma, an electrically neutral medium of unbound positive and negative particles, is created due to the ionization process. Pressure plays a key role in sustaining the plasma. If it's too low there aren't enough collisions between the sputtering gas and electrons, and if it's too high there are too many collisions where electrons don't have enough time to obtain enough energy to ionize the sputtering gas.

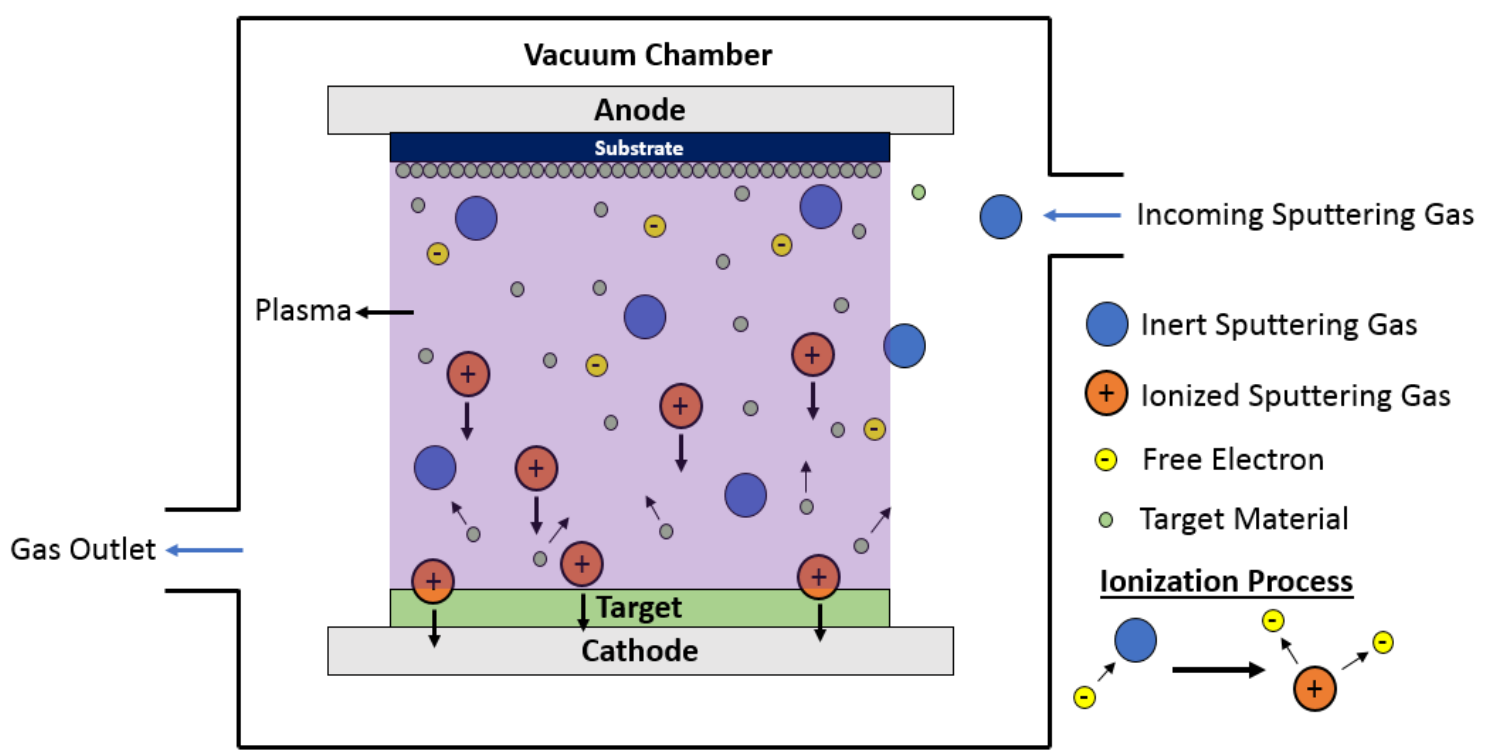

Figure 3-2: Sputtering Schematic

Elastic collisions are assumed dominant for sputtering since incident ion energies are much higher than the lattice bonding or vibrational energies in the target material which are responsible for inelastic interactions. Different ion energies dictate different 
sputtering regimes. For sputtering, there are 3 that are considered: Single knock-on regime, linear cascade regime, and the spike regime. In the single knock-on regime, atoms from the target material recoiling from the incident ions obtain enough energy to be sputtered, but not to generate recoil cascades. For the most part, energies are below $1 \mathrm{keV}$. The linear cascade regime, the recoil atoms themselves produce further recoil cascades. The amount of recoil atoms is quite low which result in infrequent collisions between atoms, therefore knock-on collisions remain dominant. Incident ion energies are between $1 \mathrm{keV}$ and $1 \mathrm{MeV}$. Higher energies (spike regime) entail a high density of recoil atoms, setting many in motion.

The number of atoms removed from the surface with respect to the incident number of ions is known as the sputter yield. This yield depends on the type of material being sputtered, the binding energy of the material, the relative mass of ions and atoms, incident ion energy, and the angle of incidence. For the most part, a typical sputter yield can range from 0.1 to 10 sputtered atoms per incident ion. The sputtering rate is clearly proportional to the sputter yield where different pressures affect the amount of collisions, ions, and scattering. Metals typically have a much higher sputter rate $(\sim 100 \mathrm{~A} / \mathrm{s})$ than insulators $(1-10 \mathrm{~A} / \mathrm{s})$. Sputtered atoms have energies between 2-7 eV and travel to the substrate via diffusion. The energy of the ejected atoms of the target material can be up to two orders of magnitude more than those thermally evaporated. This higher energy typically results in better adhesion. An important advantage of sputtering versus other PVD methods is its capability to sputter targets made up of different material compositions and keep a similar stoichiometry at the substrate. The angular distribution of the sputtered material is also a function of pressure where higher pressures result in 
more uniformity and decent step coverage, while lower pressures are more directional and less uniform.

There are several different methods to enhance the sputtering process. A very common method is by adding a strong magnetic field near the target area, creating what is known as a magnetron sputtering system. The field traps travelling electrons causing them to spiral in the magnetic flux lines near the target instead of going directly to the substrate. Given the proximity of the field lines to the cathode, plasma is generated and confined near the target. This confined plasma reduces the chance of damaging the film sputtered on the substrate and allows the sputtering process to be done at lower pressures. This setup also enables electrons to travel farther, increasing the chance to ionize the sputtering gas, thus making the sputtering process more efficient. Magnetron sputter guns generally have a planar configuration embedded with high quality permanent rare-earth magnets that have high curie temperatures to create the magnetic fields. This planar configuration involves a concentric setup, with the center being occupied with a magnet with one polarity (ex: North) and the perimeter having magnets with the opposite polarity (South). Because of the magnetic configuration, targets are eroded much faster in areas where the field is parallel to the target. 


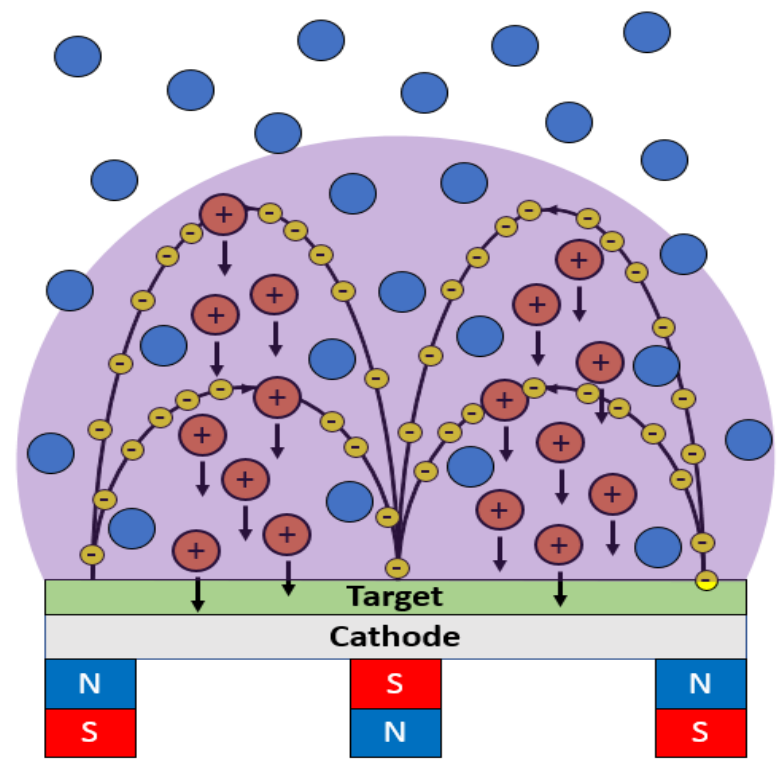

Figure 3-3: Magnetron Sputtering Process

The system used to deposit the films for the MTJs is an AJA ATC Orion sputter system with three 2" A300-XP magnetron sputter sources, ultra-high vacuum (UHV) capability, rotating substrate stage, heating, and a load-lock. The sources are placed in the bottom pointing up towards the substrate for what is known as a "sputtering up" configuration, since the sputtered material travels from the bottom of the sputtering chamber to the top. Both argon and oxygen can be introduced into the chamber and their flow is regulated using mass-flow-controllers (MFC). The chamber is initially pumped down using a roughing pump, where the chamber is exposed to atmosphere and proceeds through the viscous regime. In this regime, the gas molecules have a mean-free-path (MFP) shorter than the dimensions of the chamber and are therefore colliding constantly with each other. The system is pumped via viscous flow from atmosphere down to $\sim 10^{-3}$ Torr. As the MFP is increases, the gas particles begin to flow independently of a pressure gradient, called the molecular regime. In this regime, the AJA system is equipped with a 
turbomolecular pump that brings the pressure down to $\sim 10^{-8}$ Torr. A "getter," such as titanium and chromium, can be sputtered inside the vacuum chamber to absorb background gas and assist in the pump down. A full range gauge (atmosphere to $\sim 10^{-9}$ Torr) is used to establish the base pressure of the chamber.

Depending on the type of material that needs to be sputtered, different power sources and gun configurations must be considered. When sputtering non-magnetic conducting materials, a DC power supply is used where the plasma is struck and maintained with a DC current/voltage bias. Should we sputter an insulating material, the target will charge and repel Ar ions in the plasma. We must use a Radio Frequency (RF) power supply. RF sputtering operates using an $\mathrm{AC}$ voltage where sputtering occurs on the negative swing of the signal, and accumulated ions are neutralized during the positive swing. The RF power supply operates at a frequency of $13.56 \mathrm{MHz}$, a frequency reserved exclusively for industrial and laboratory processes. Impedance matching is needed when using RF so a matching network is embedded into the AJA system. Monitoring the reflected and forward power is of the upmost importance since a poor impedance match could result in power dissipation in the transmission line or in the power supply. RF sputtering can also be used to sputter conducting materials but for the devices made in this thesis it was just used for non-conductors. Finally, the sputtering gun must be configured accordingly to deposit magnetic materials since they alter the fields of the magnetron gun. One of the three guns in the AJA system is configured to sputter magnetic materials by removing the permanent magnet in the middle of the gun and replaced with a copper piece.

When characterizing the films for the MTJ, the main considerations were the processing pressure and the power. In magnetron sputtering, the confinement of the plasma allows 
for a wide variation of processing pressure. Films were deposited from pressures ranging from $0.5 \mathrm{mTorr}$ to $20 \mathrm{mTorr}$ using a capacitance diaphragm gauge (CDG), which is accurate from $0.1 \mathrm{mTorr}$ to $100 \mathrm{mTorr}$. This pressure range was chosen in accordance to the Thornton Zone Model that provides insight to the relation between thin film morphology vs. pressure and substrate temperature.

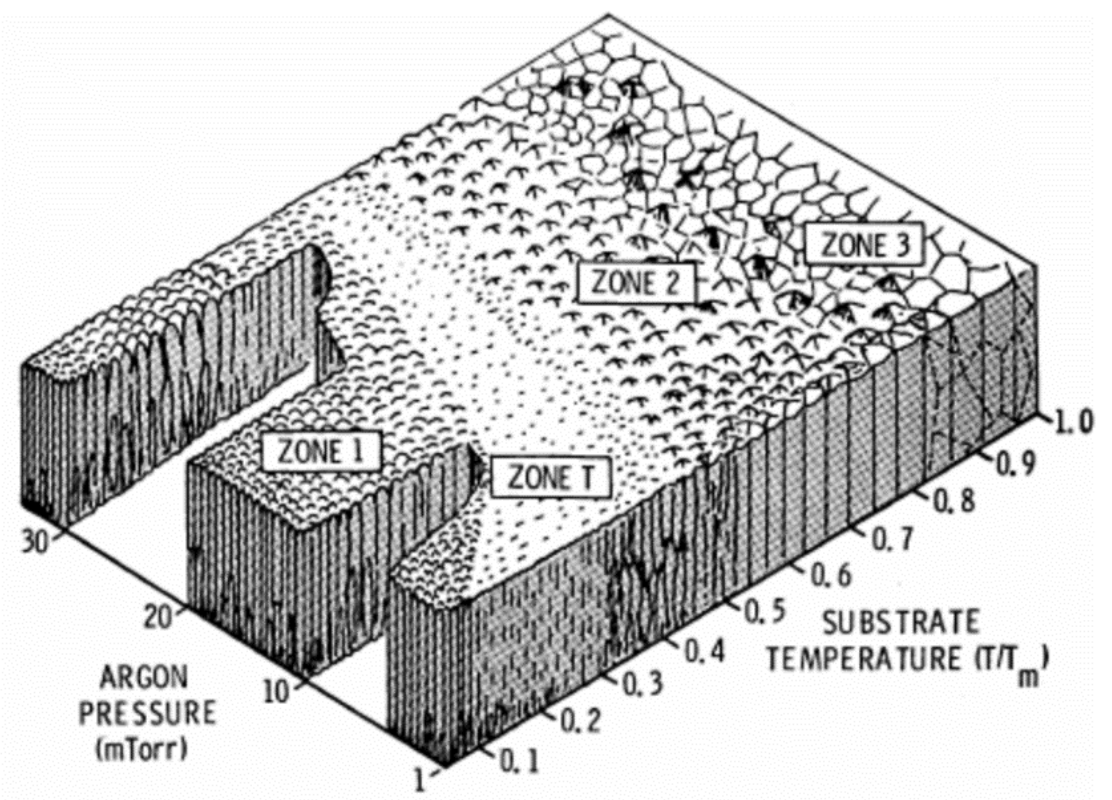

Figure 3-4: Thornton Zone Model [42]

Zone 1 is rather porous and rough, an unwanted characteristic for MTJs. Zone $\mathrm{T}$ is most suitable because is consists of smooth and densely packed grains. Zone 2 and 3 are not considered since they occur at pressures and temperatures beyond the capability of the sputtering equipment. The roughness of the films was measured using AFM and the deposition rate was recorded by using both AFM and profilometry. There is a clear trade-off between the sputtering rate, roughness and uniformity with the operating pressure. At higher pressures $(>10 \mathrm{mT})$ films were uniform but rough and sputtered at slower rates. Too low pressure $(<1 \mathrm{mT})$ resulted in non-uniformity, although the rates were higher and the films were smooth. There was also the concern that at too low of a 
pressure, the ejected material can gain enough kinetic energy because of the longer MFP and lodge themselves in the tunnel barrier.

The power chosen depended on the material sputtered. Typically, a minimum of $\sim 15$ Watts is needed to strike and maintain a plasma. For most metals, studies were done from 25-150 Watts. $\mathrm{MgO}$ and $\mathrm{Al}_{2} \mathrm{O}_{3}$ were carefully deposited at 200 Watts since the sputtering rate is really low. Given the low thermal conductivity of most insulators, the power was gradually ramped up and down during runs. For long runs (>30min), targets were left to cool periodically. Typically, higher sputter rates yield smoother films but using too much power can damage the target due to thermal stress. The guns in the AJA system are not tilted, therefore to ensure uniformity across the sample the substrate had to be rotated. Rates were also chosen to ensure sufficient rotation of the sample. Prior to any deposition, targets were "pre-sputtered" onto the shutter for several minutes to get rid of any surface contamination and unwanted films. The following table shows the optimized parameters for the sputtered films used in the development of the MTJ devices.

Table 3-1: Sputtering Parameters

\begin{tabular}{|l|l|l|l|l|}
\hline Material & $\begin{array}{l}\text { Power } \\
\text { (Type/Watts) }\end{array}$ & $\begin{array}{l}\text { Pressure } \\
\text { (mTorr) }\end{array}$ & $\begin{array}{l}\text { Gas Flow } \\
\text { (Gas/sccm) }\end{array}$ & $\begin{array}{l}\text { Deposition } \\
\text { Rate }(\mathbf{A} / \mathbf{s})\end{array}$ \\
\hline $\mathrm{Ti}$ & $\mathrm{DC}, 70$ & 5 & $\mathrm{Ar} / 10$ & 0.33 \\
\hline $\mathrm{Cr}$ & $\mathrm{DC}, 70$ & 10 & $\mathrm{Ar} / 10$ & 0.71 \\
\hline $\mathrm{Au}$ & $\mathrm{DC}, 125$ & 5 & $\mathrm{Ar} / 10$ & 5 \\
\hline $\mathrm{Ta}$ & $\mathrm{DC}, 40$ & 5 & $\mathrm{Ar} / 10$ & 0.33 \\
\hline $\mathrm{CoFeB}$ & $\mathrm{DC}, 75$ & 5 & $\mathrm{Ar} / 10$ & 0.83 \\
\hline $\mathrm{MgO}$ & $\mathrm{RF}, 200$ & 5 & $\mathrm{Ar} / 10, \mathrm{O}_{2} / 1$ & 0.18 \\
\hline $\mathrm{Al}_{2} \mathrm{O}_{3}$ & $\mathrm{RF}, 200$ & 5 & $\mathrm{Ar} / 10, \mathrm{O}_{2} / 1$ & 0.16 \\
\hline $\mathrm{Co}$ & $\mathrm{DC}, 70$ & 5 & $\mathrm{Ar} / 10$ & 0.8 \\
\hline $\mathrm{Pd}$ & $\mathrm{DC}, 40$ & 5 & $\mathrm{Ar} / 10$ & 0.3 \\
\hline
\end{tabular}




\subsubsection{Evaporation}

Electron beam or "E-Beam" evaporation is another physical vapor deposition technique used in the fabrication of the MTJ devices in this thesis. As the name implies, an electron beam is generated from a charged tungsten filament and guided onto the source material using electric and magnetic fields. The electron beam bombards and heats the surface of the material, converting it into a gaseous state where the atoms precipitate along the chamber walls and substrate. This deposition technique is line-of-sight. E-beam evaporation must be operated at a high vacuum $\left(\sim 10^{-6}\right)$ so that electrons from the filament arrive unimpeded to the source material. Since it's a thermal process, the atoms released arrive at the substrate with energies less than $1 \mathrm{eV}$. Sputtered material have energies that are several orders of magnitude higher than evaporated material, but lower energies will be less destructive to the substrate. Since e-beam deposition is "line-of-sight," it's an ideal process for lift-off but not for ensuring proper step coverage. This PVD method is limited to elements, and alloys and compounds that have constituents with similar vapor pressures. 


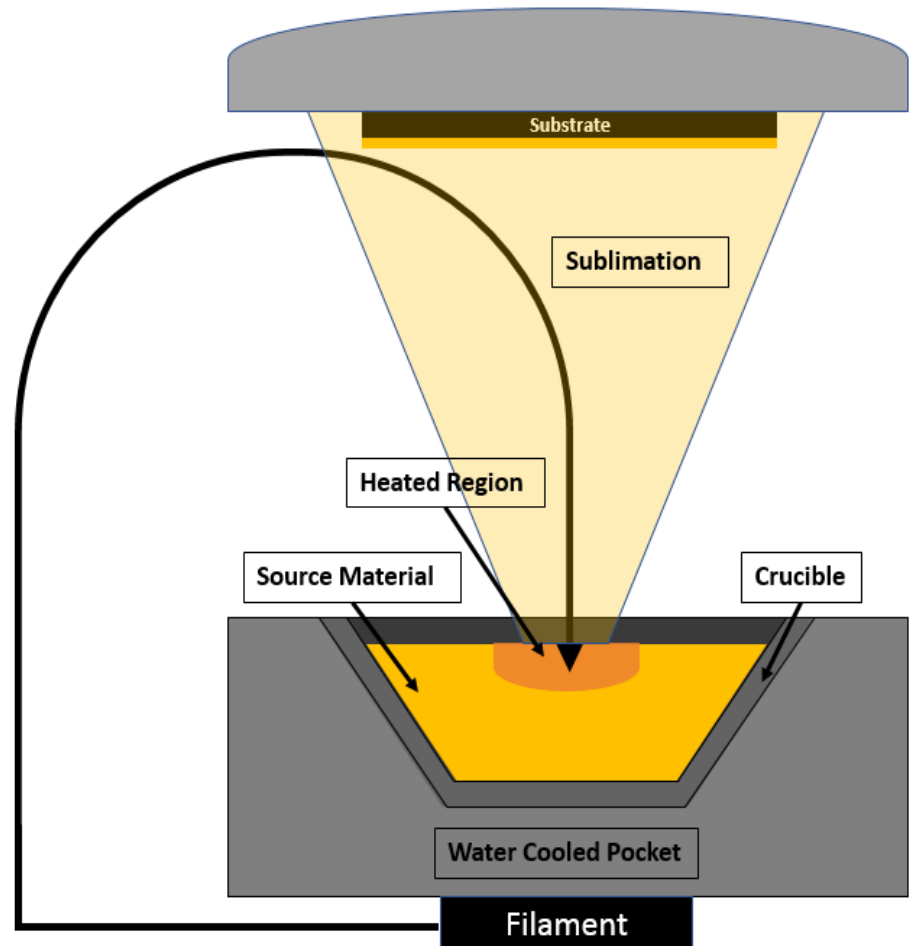

Figure 3-5: E-Beam Evaporation Schematic

A CHA E-Beam Evaporator was used to develop MTJ devices. This system can evaporate up to three materials without breaking vacuum since it has 3 rotatable pockets. Each pocket is water-cooled and can hold a 20cc crucible. This system is equipped with a roughing pump and a cryogenic pump capable of reaching a base pressure of around $\sim 10^{-}$

${ }^{7}$ Torr. The system is interfaced with a PID controller, and the film thickness is monitored with a quartz crystal sensor. The e-beam evaporator was mainly used to deposit electrodes. Typical materials deposited in the evaporator are titanium, chromium, nickel, iron, and gold. Samples can be loaded on the ceiling of the vacuum chamber or onto a rotating planetarium. This planetarium can be rotated at different speeds allowing for adequate step coverage if needed. 


\subsection{Lithography}

Lithography is an important miniaturization method used to transfer patterns onto a substrate. This process allows to structure material at a fine scale, where different lithographic methods can yield different size resolutions. Lithographic techniques typically involve a radiation source (ex: photons, x-rays, etc.) that expose a sacrificial polymer sensitive to that radiation. There are many different types of lithography, but in this section the main focus will be on Photolithography. This patterning technique is a two-dimensional process with limited capabilities for extreme topographies.

\subsubsection{Photolithography}

This form of lithography is the most widely used in microfabrication where patterns are transferred from a mask onto a substrate. Photolithography uses light, typically in the UV spectrum, to expose a light sensitive polymer called photoresist on the substrate. This process is also known as optical lithography or UV lithography. Due to the sensitivity of photoresist to wavelengths $<500 \mathrm{~nm}$, photolithography must be done in labs with yellow light. A clean-room environment is necessary to avoid defects due to particles settling on the substrate and mask. The photolithography in this thesis was done in a Class 100 cleanroom (less than 100 particles $\geq 5$ um per $\mathrm{ft}^{3}$ ), although upon recent inspection, it has been found to have less than 10 particles $\geq 5$ um per $\mathrm{ft}^{3}$, deeming it a Class 10 . The following is a set of process steps illustrating the pattern transfer:

\section{Wafer Preparation}

This is the initial step where any contaminants (organic and inorganic) are cleaned off of the substrate. Surface contamination can affect both the adhesion of the photoresist spun on the wafer and alter the pattern exposure. With new unprocessed wafers, a simple 
solvent clean is sufficient, otherwise a full RCA clean is recommended. The following figure is a substrate with a thin film deposited on it, ready to go through the miniaturization process.

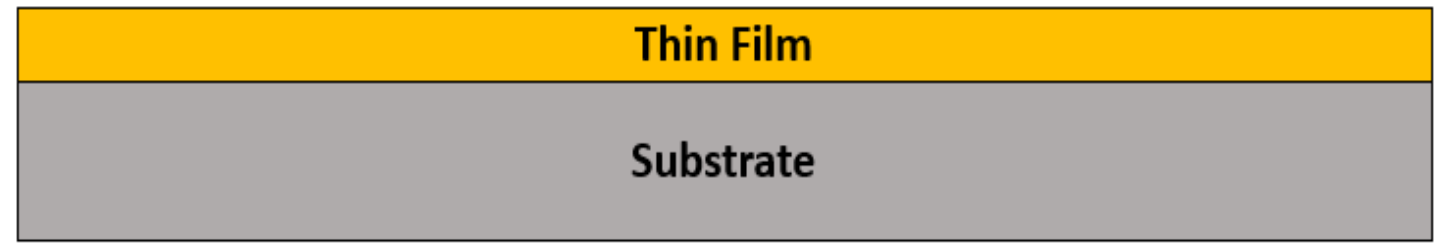

Figure 3-6: Step 1 Wafer Preparation

\section{Lithography Preparation}

Before putting down the photoresist a dehydration bake is done. This bake drives moisture off the surface of the wafer to promote adhesion. Wafers are placed in ovens or on hotplates with temperatures between $115-150{ }^{\circ} \mathrm{C}$ for $5-10$ minutes. Photoresist is a nonpolar substance and therefore adhesion is better achieved on hydrophobic surfaces. Hydrophilic surfaces have polar $\mathrm{O}-\mathrm{H}$ bonds that prevent the wetting of the resist. If necessary, to further improve adhesion wafers can be primed with hexamethyldisilazane (HMDS). This adhesion promoter is not a necessary step but it is typically used when patterning on top of oxides, nitrides, polysilicon, glass, quartz, and any other difficult hydrophilic surfaces.

The wafer is covered with photoresist via spin-coating. Photoresist is a thin, organic polymer sensitive to UV radiation. Spin coating is a standard procedure that deposits uniform thin films on flat substrates. Centrifugal forces are responsible for the coating since photoresist is dispensed at the center of the substrate which is rotated at high speeds (1500-8000 rpm). The polymer solution flows to the edge of the wafer where it builds up 
until surface tension is exceeded. The thickness of the resist $(\mathbf{T})$ can be expressed with the following empirical expression as:

$$
T=\frac{K C^{\beta} \eta^{\gamma}}{\omega^{\alpha}}
$$

$\mathbf{K}=$ calibration constant, $\mathbf{C}=$ polymer concentration, $\boldsymbol{\eta}=$ polymer intrinsic viscosity, $\boldsymbol{\omega}=$ rotation of sample. The parameters $\boldsymbol{\alpha}, \boldsymbol{\beta}$, and $\boldsymbol{\gamma}$ are exponential factors that need to be determined experimentally to predict the thickness of the polymer. Manufacturers of photoresist usually provide spin curve graphs that relate the spin-speed with the polymer thickness.

After you spin the wafer, it's important to check for spinning artifacts such as striations and streaks. These effects are generally due to cleanliness of the substrate (particles) and insufficient spinning of the resist. A major artifact to watch out for is the edge bead. This is a buildup of resist at the edge of the wafer that can be 20-30 times the intended thickness of the resist. There are certain solvents and techniques available to get rid of the edge bead.

Once inspected, the substrate is baked to remove solvents, relieve built in stress in the polymer, and promote adhesion. This step is known as the "soft-bake" and is done at temperatures typically between $90-110{ }^{\circ} \mathrm{C}$ for around 60 seconds. This step is critical for device yield. If not sufficiently baked, solvents won't be completely removed. If baked too much, the photoactive compounds can be destroyed, reducing sensitivity. 


\section{Photoresist}

\section{Thin Film}

\section{Substrate}

Figure 3-7: Step 2 Lithography Preparation

\section{Pattern Transfer via Exposure}

When the soft bake is done, the wafer is exposed to UV light. A pattern is generated on the wafer by using a photo-mask. A photomask is typically made of glass with a pattern defined by an absorber-metal film. Depending on the tone of the resist, light field or dark field masks are used. In a dark field mask, the pattern is transparent and vice versa for a light field one. Positive tone resists when exposed to UV light will weaken the polymer making it soluble in developing solutions. Negative tone resists will strengthen from cross-linkage when exposed, becoming less soluble to developing solutions. The process illustrated in these steps is an example of the use of a positive tone resist.

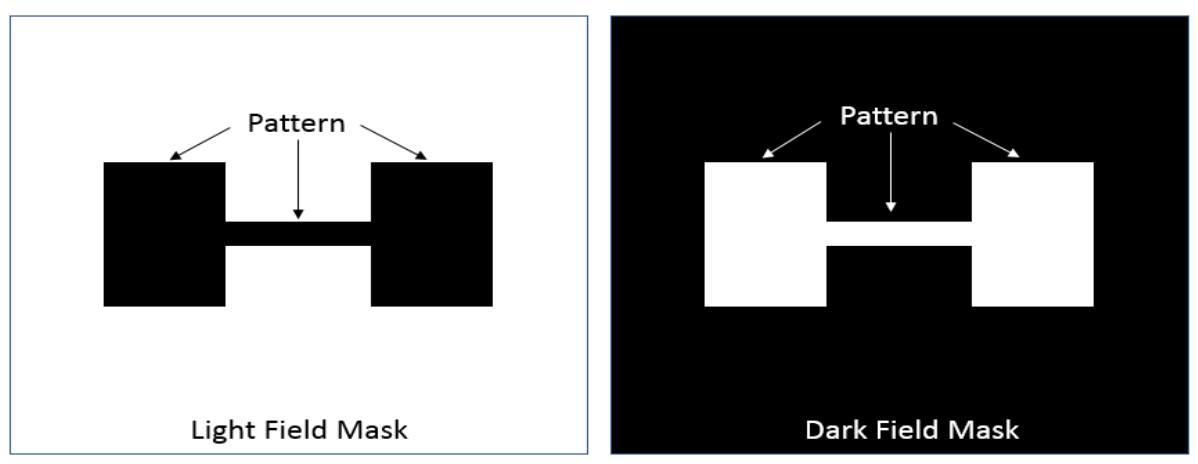

Figure 3-8: Light Field and Dark Field Masks

In photolithography, wavelengths in the UV spectrum include extreme ultraviolet (EUV, $\lambda=10-14 \mathrm{~nm}$ ), deep ultraviolet (DUV, $\lambda=150-300 \mathrm{~nm}$ ), and near ultraviolet (UV, $\lambda=$ $350-500 \mathrm{~nm})$. The resists used for the devices developed here were exposed to UV from 
an OAI Mask Aligner using the g-line (435nm), h-line (405nm) and i-line (365nm) of a mercury lamp. Manufacturers will also provide the dose required to expose the photoresist. The time of required illumination is a function of the resist material (exposure dose) and the intensity of the light source (Dose $=$ Intensity $\times$ Time). Depending on the photoresist recipe, a post-exposure bake is done to reduce standing waves and thermally activate the chemical process.

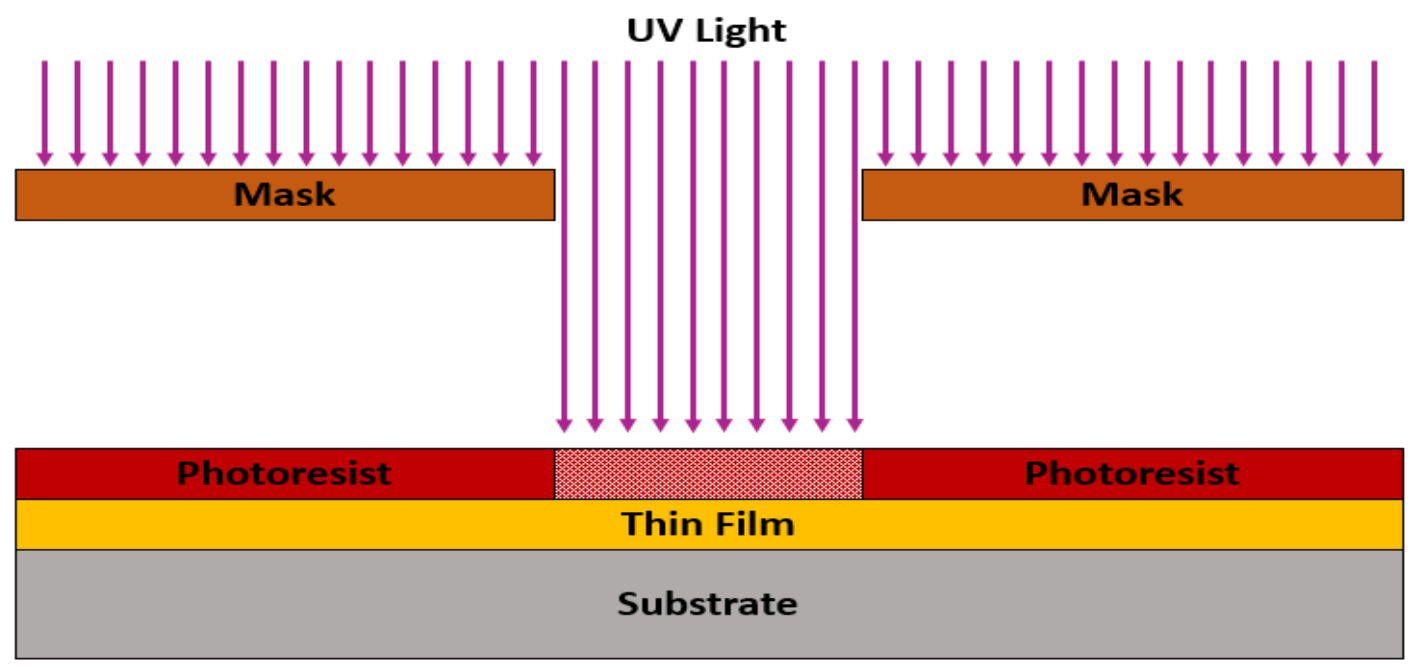

Figure 3-9: Step 3 Pattern Transfer

\section{Development}

After the bake, the next step is development. Soluble parts of the resist are etched away while the non-soluble parts remain. Once this step is completed, the pattern from the mask is visible on the wafer, which in turn will serve as a mask for any future additive or subtractive processes. Development parameters, including the recommended solution to use, are usually provided by the manufacturer. Many developers contain sodium hydroxide $(\mathrm{NaOH})$, but the sodium ions were found to degrade the insulating properties of oxides because they would migrate into them. For our devices, it is preferable to use metal-ion-free developers, such as tetramethylammonium hydroxide (TMAH). Different 
concentrations of the solution and temperature will affect the rate of development. This process is highly selective, but if there are critical features it's important to fully optimize the development process.

Development can be done by immersion or spray. Immersion development was done for the photolithography steps in this thesis. It's a simple process that involves immersing the wafer in a bath of developer, followed by a rinsing and cleaning process. Spray development is a preferred method since the chemicals are always clean and the spray pressure usually improves image definition. This process is usually embedded into the resist coater and spin dryer. Once developed, the wafer is inspected where we check the resolution of the pattern, misalignment, surface contamination, and pattern distortion. If there are any problems the process must be done all over again, beginning with stripping the resist off and cleaning the substrate.

Once inspected, a brief oxygen plasma treatment can be done to remove any unwanted resist still left after development. This step is known as "de-scumming." Furthermore, a final baking process called "hard baking" can be done to remove residual solvents and strengthen the adhesion. This baking step is invaluable if there is processing that involves etching since the photoresist will be hardened, increasing the selectivity of the process. Hard baking is usually done between $120-180{ }^{\circ} \mathrm{C}$ for 20 minutes.

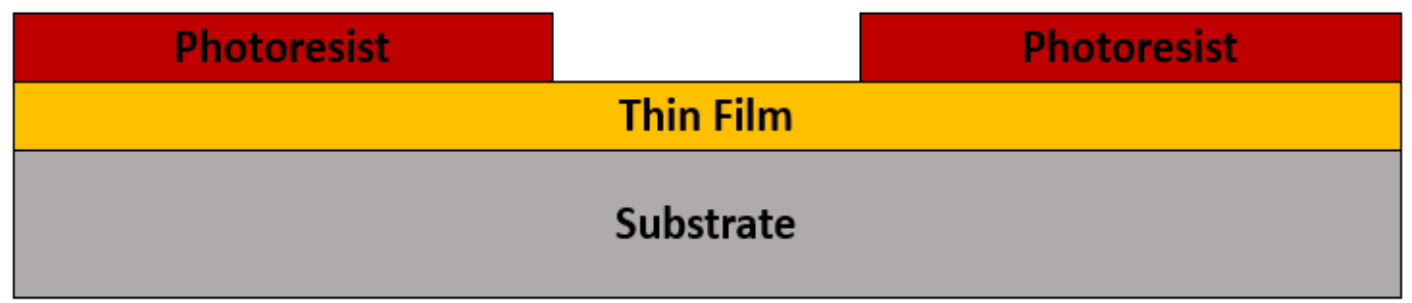

Figure 3-10: Step 4 Development 


\section{Material Process}

The photolithographic step is complete and the wafer can undergo further processing. It's important to understand that any future processing must consider the photoresist used, whether it be additive (ex: metal deposition) or subtractive (ex: plasma etching). There are different photoresists and lithographic variations that cater to different processes. In the illustration below, the metal film deposited is etched where the photoresist is not present.

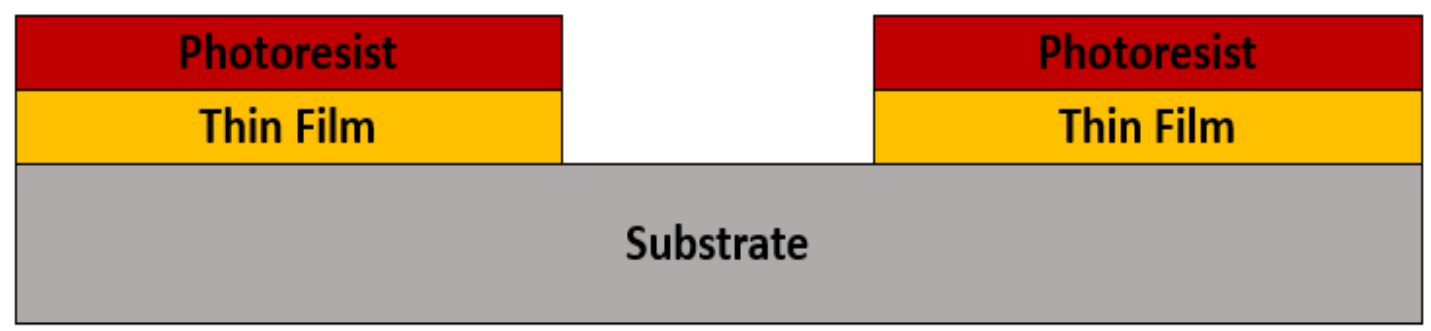

Figure 3-11: Step 5 Material Processing

\section{Remove Resist}

Once the processing is done the photoresist is no longer needed and must be stripped off. Solvents such as acetone and trichloroethylene (TCE) can be used for samples that weren't hard baked for a long time. Otherwise, liquid strippers and alkaline strippers can be used. Before using and solvents or strippers, compatibility with the substrate and any films on it must be considered. Oxygen plasma stripping or "ashing" is a more controlled process that can be less corrosive and ideal for removing organic polymer debris. The photolithographic process is complete. 


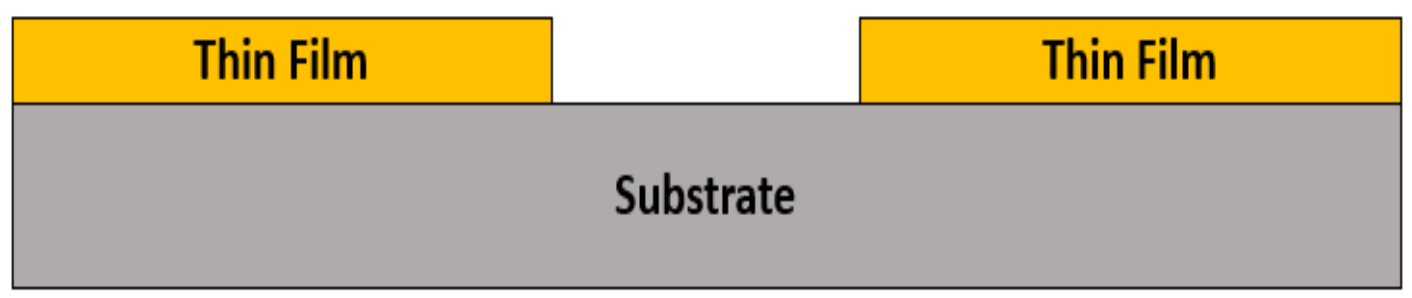

Figure 3-12: Step 6 Remove Resist

Photoresists are usually made up of 3 components: Resin (a polymer), which gives the resist structure; Solvents, responsible for the thickness of the resist; and photo active compounds (PAC) which is the light sensitive component.

Negative tone resists were the first ones to be used in the semiconductor industry. The resist becomes insoluble in developer solution when exposed to UV light. The first negative resists used UV radiation to increase the molecular weight, rendering them less soluble. Newer resists generate insoluble products by photochemical transformations. Overall, the resist becomes polymerized through a process called "cross linking." Since cross-linking is a process that starts from the top, the resolution of the photoresist is limited by the film thickness. It is important to note that the thicker the resist the longer the required exposure (longer scattered radiation). Furthermore, the developer swells the cross-linked resist hindering its size resolution even more. Organic solutions, such as benzene, are used for development. Despite the issues with size resolution, negative resists for the most part have great adhesion to many substrates and are highly resistant to acid and alkaline solutions, and oxidizing agents.

Positive tone resists when exposed the photochemical reaction weakens the polymer by breakage of chemical bonds or "scission." This makes the exposed polymer much more soluble in developing solutions. Two main families of positive resists can be discerned 
based on certain components. 1) Poly(methylmethacrylate) (PMMA) is a component for positive resists that operate under DUV. 2) The other is a 2-component DQN that is composed of the photoactive diazoquinone ester (DQ) and a phenolic novolak resin. This resist operates in the NUV spectrum and is one the most used. The mask aligner used in photolithography for this thesis has a light source that radiates in the NUV; therefore any positive tone resists used are part of the DQN family. The main advantage of positive resist over negative is that they do not swell during development since unexposed regions are permeated by developer solution. Alkalis such as $\mathrm{NaOH}$ are used for development. Positive resists are capable of higher resolution and more resistant to plasma processes than negative resists.

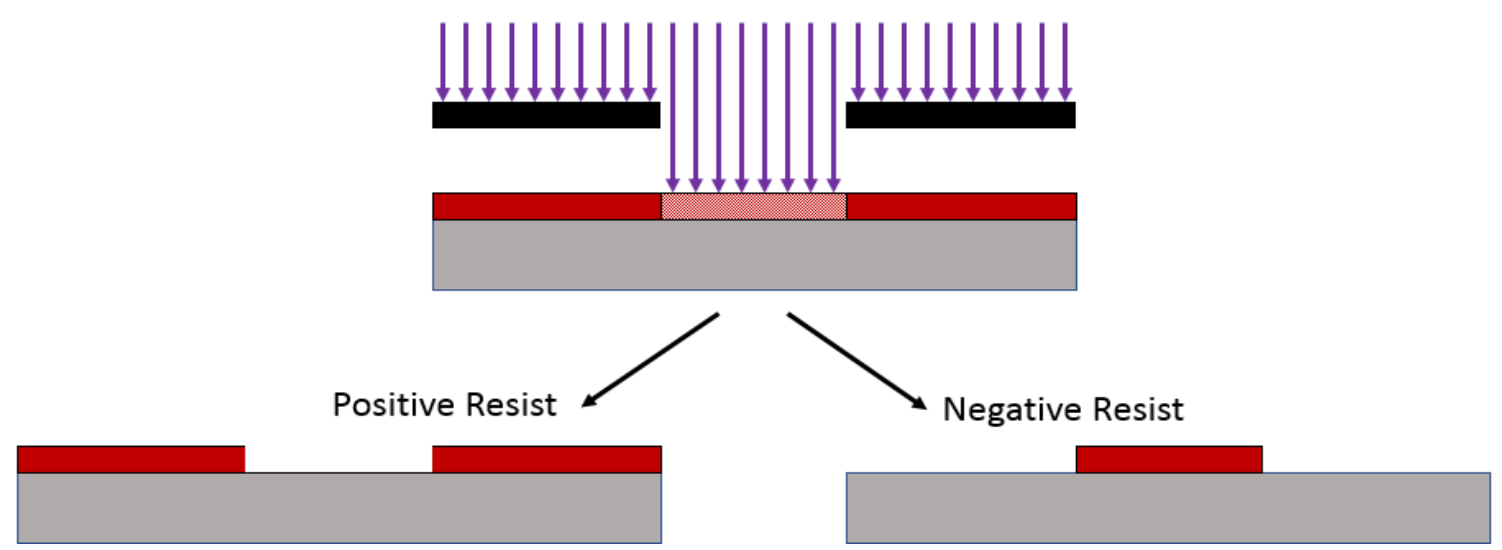

Figure 3-13: Negative and Positive Resist Exposure

An important property of the resist that must be considered is its profile. Three main profiles can be achieved: Overcut, Vertical, and Undercut. Each profile has certain advantages and disadvantages.
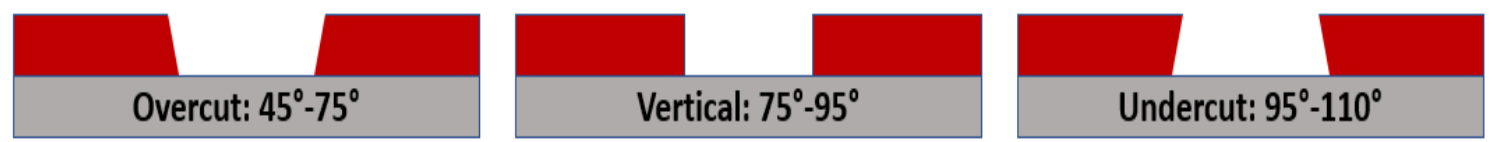

Figure 3-14: Photoresist Profiles 
An undercut profile is desirable for lift-off, an additive process that allows films to be patterned onto a substrate. Lift-off is an alternative to etching, and is one of the most used processes in the devices developed in this thesis. For proper lift-off, a gap between the deposited film and the photoresist sidewall is necessary so that the solvent used to dissolve the resist without any obstacles after the deposition. An under-cut profile is difficult to achieve with positive photoresist since the UV exposure is greater at the surface than at the interface between the resist and the wafer. With negative resists, this profile is easily obtained for the same reason as positive resists; crosslinking begins at the surface and works its way down. Should a user want to use positive resist, the standard method is to utilize a double layer of positive resists, where the layer at the substrate/resist interface develops quicker than the layer on top. Inadequate undercut for lift-off will result in an undesirable "wings" at the edges of the patterned metal due to sidewall coating. An overcut and vertical profile is typical for positive resists and they are more suitable for etch processes.

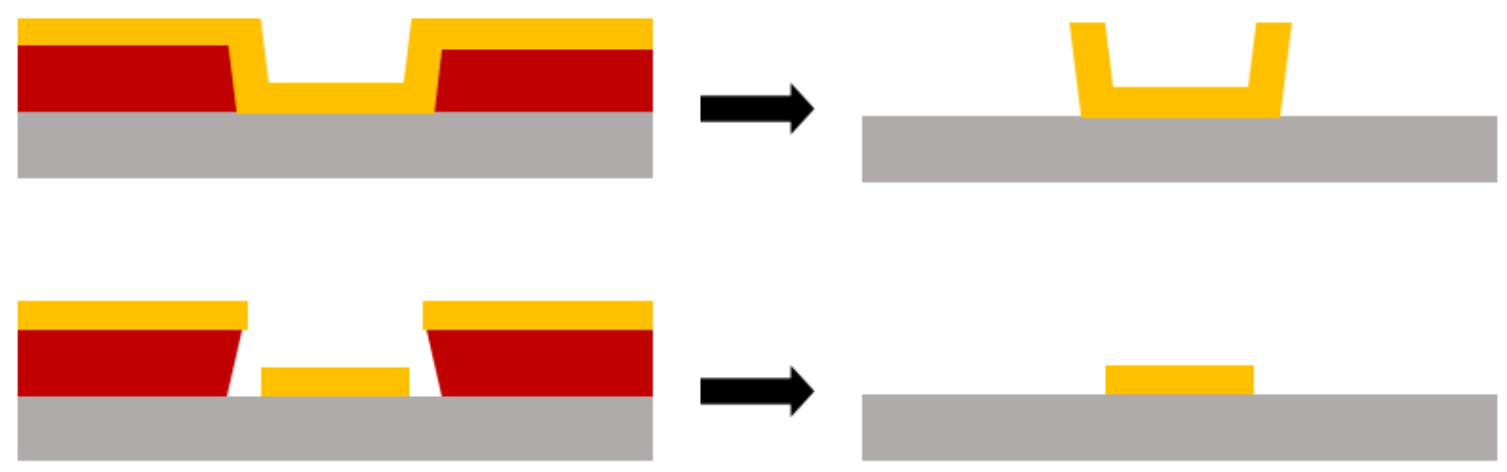

Figure 3-15: Lift-Off with Overcut (top) and Undercut (bottom) Sidewall Profiles Aside from bilayers, another method to utilize positive resist for lift-off is called "image reversal." The process begins by exposing the photoresist with UV light. The exposed pattern becomes soluble as would any positive resist. A "reversal bake" takes place where 
the exposed area becomes cross-linked while the unexposed potion remains photoactive. This bake promotes diffusion of an amine vapor (such as imidazole or triethanolamine) neutralizing the byproduct of the photodecomposition. Finally, the full sample is exposed to UV radiation, a step called "flood exposure," making the previously unexposed region soluble in developing solution. This offers unprecedented advantages: the undercut profile typical of negative resists and the resolution of positive resists. Without the reversal bake, the resist has the same attributes as a positive resist. These image reversal resists are also referred to as "dual-tone" resists. AZ 5214-IR is an image reversal resist used extensively in the development of MTJs.

The "critical dimension" (CD) is the minimum feature size that can be consistently resolved in lithography. It is a function of many aspects of the process such as the mechanical stability of the hardware used, material properties, scattering of the light, etc. This resolution is quantified by "line-width" measurements. The "line-width" is the distance between two resist-air boundaries. Besides the actual size of the feature, in IC manufacturing it is also important to see how close we can bring devices together. These measurements can be measured by looking at the "half-pitch," or the space between two line-widths. Lithography can be done using contact, proximity, or projection.

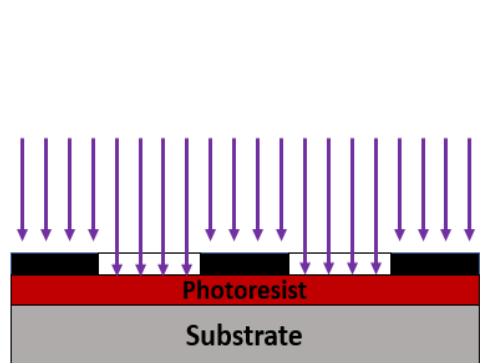

Contact Lithography $(s=0)$

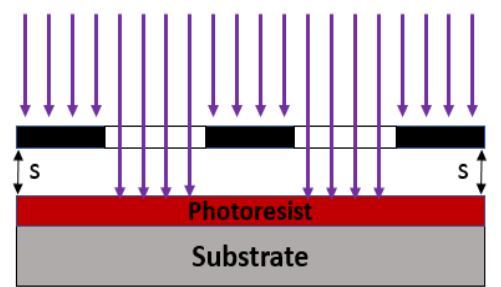

Proximity Lithography $(s \neq 0)$

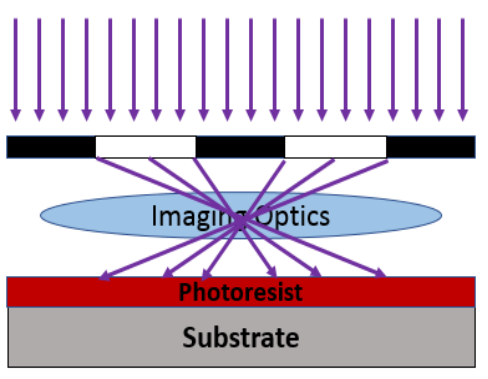

Projection Lithography

Figure 3-16: Types of Photolithography 
Projection lithography patterns the wafer by scanning and projecting the images of the mask. It is typical in IC manufacturing where the minimum feature size $(\boldsymbol{C D})$ can be approximated by the following formula: $C D=k_{1} \frac{\lambda}{N A}$, where $\boldsymbol{k}_{\boldsymbol{1}}$ is an experimental parameter that reflects process related factors and $N A$ is the numerical aperture of the lens as seen by the substrate. This equation is in direct relation with the Rayleigh criterion. Resolution can be improved by using smaller wavelengths and increasing the numerical aperture. While it may seem straight forward, changing these parameters to improve resolution comes at the cost of depth of focus $\left(\boldsymbol{D}_{F}\right)$. Depth of focus can be expressed as $D_{F}=\frac{\lambda}{N A^{2}} k_{2}$ where $\boldsymbol{k}_{2}$ is another process related coefficient. To accommodate this change in depth of focus, the thickness of the resist and surface topologies must be addressed. This process is very expensive but there is no mask wear/contamination, improving yield. Due to the cost, this method is not used in this thesis.

Contact and proximity lithography involve placing the mask in direct or close contact with the surface of the photoresist. An important factor to consider in the resolution of this method (in addition to surface topography and particles) is the diffraction of the light on the opaque edges of a pattern. The following figure shows the distribution of light intensity on the surface of the photoresist after passing through a periodic grating. 


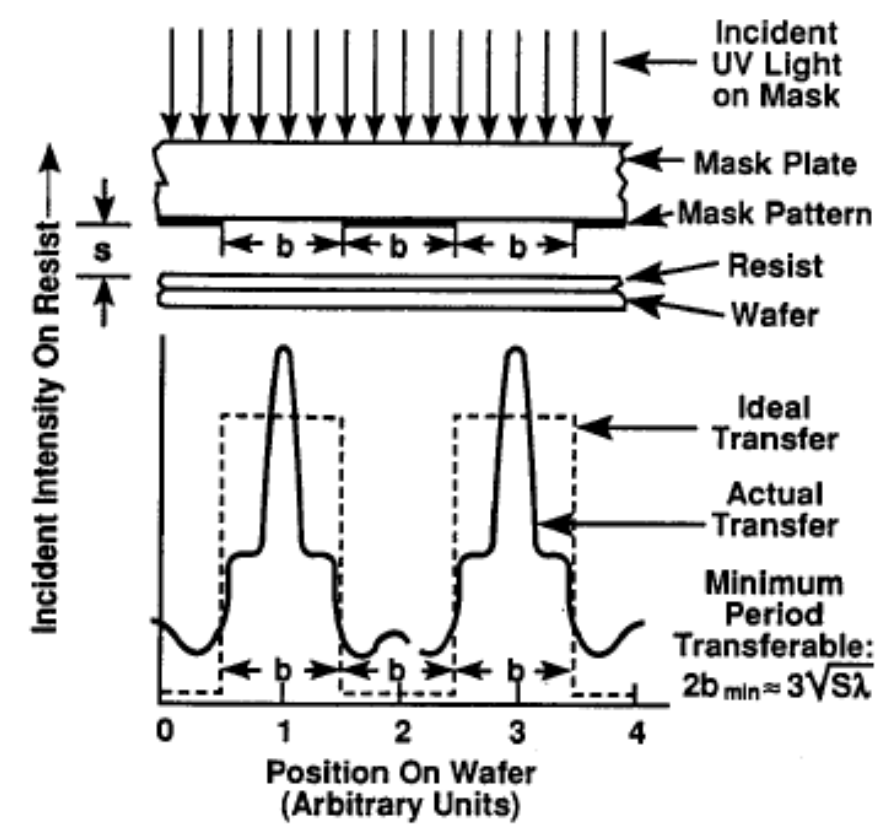

Figure 3-17: Light Intensity Profile on Mask with Grating [42]

Diffraction at the edge of the pattern clearly plays a major role in the resolution. Contact and proximity printing have a theoretical resolution:

$$
b_{\text {min }}=\frac{3}{2} \sqrt{\lambda\left(s+\frac{Z}{2}\right)}
$$

The minimum feature resolution $\boldsymbol{b}_{\min }$ is half the grating period, $\lambda$ is the wavelength of light, $s$ is the gap between the mask and the resist, and $z$ is the thickness of the resist. The main issue with this method is the wear and tear on the mask. The closer we place the mask to the surface of the resist the higher the resolution we can achieve, but at the expense of further degradation of the mask. The maximum resolution requires direct contact, making $(s=0)$. To further improve resolution, smaller wavelengths $(\lambda)$ could also be used (ex: x-rays). This method of lithography is mostly used in R\&D because it is simple and requires relatively inexpensive equipment. Contact lithography is the method of choice in the development of MTJ devices in this thesis. 
In the process of fabricating the MTJs, several different photoresists were characterized:

For lift-off:

1. $\underline{\mathrm{AZ}}$ nLOF 2020 (Negative Tone)

Table 3-2: AZ nLOF 2020 Recipe

\begin{tabular}{|l|l|}
\hline Dehydration Bake & Hotplate $5 \mathrm{~min} / 120^{\circ} \mathrm{C}$ \\
\hline Spin Coat & Resist Thickness: $2 \mathrm{um}$ \\
& Spin 1: $500 \mathrm{rpm}$ \\
& Ramp 1: $250 \mathrm{rpm} / \mathrm{s}$ \\
& Time 1: $5 \mathrm{~s}$ \\
& Spin 2: $3000 \mathrm{rpm}$ \\
& Ramp 2: $3000 \mathrm{rpm} / \mathrm{s}$ \\
& Time 2: $30 \mathrm{~s}$ \\
\hline Soft Bake & Hotplate $1 \mathrm{~min} / 110^{\circ} \mathrm{C}$ \\
\hline Exposure & $66 \mathrm{~mJ}$ \\
\hline Hard Bake & Hotplate $1 \mathrm{~min} / 110^{\circ} \mathrm{C}$ \\
\hline Development & AZ400K $4($ DI-Water $): 1($ Developer $)$, \\
\hline Descum & $180 \mathrm{~s}$ \\
\hline
\end{tabular}

2. $\underline{\mathrm{AZ}} 5214$ IR (Negative Tone IR)

Table 3-3: AZ 5214-IR Negative Tone Recipe

\begin{tabular}{|l|l|}
\hline Dehydration Bake & Hotplate $5 \mathrm{~min} / 120^{\circ} \mathrm{C}$ \\
\hline Spin Coat & Resist Thickness: $1.2 \mathrm{um}$ \\
& Spin 1: $500 \mathrm{rpm}$ \\
& Ramp 1: $250 \mathrm{rpm} / \mathrm{s}$ \\
& Time 1: $5 \mathrm{~s}$ \\
& Spin 2: $5000 \mathrm{rpm}$ \\
& Ramp 2: $2500 \mathrm{rpm} / \mathrm{s}$ \\
& Time 2: $30 \mathrm{~s}$ \\
\hline Soft Bake & Hotplate $1 \mathrm{~min} / 110^{\circ} \mathrm{C}$ \\
\hline Exposure & $60 \mathrm{~mJ}$ \\
\hline Reversal Bake & Hotplate $1 \mathrm{~min} / 120^{\circ} \mathrm{C}$ \\
\hline Flood Exposure (No Mask) & $400 \mathrm{~mJ}$ \\
\hline Development & $300 \mathrm{MIF}$ Full Concentration, $40-50 \mathrm{~s}$ \\
\hline Descum & $\mathrm{O}_{2}$ Plasma Etch for $60 \mathrm{~s}$ \\
\hline
\end{tabular}


For etching:

\section{3. $\underline{\text { AZ } 1512}$ (Positive Tone)}

Table 3-4: AZ 1512 Recipe

\begin{tabular}{|l|l|}
\hline Dehydration Bake & Hotplate $5 \mathrm{~min} / 120^{\circ} \mathrm{C}$ \\
\hline Spin Coat & Resist Thickness: $1.4 \mathrm{um}$ \\
& Spin 1: $500 \mathrm{rpm}$ \\
& Ramp 1: $250 \mathrm{rpm} / \mathrm{s}$ \\
& Time 1: $5 \mathrm{~s}$ \\
& Spin 2: $3000 \mathrm{rpm}$ \\
& Ramp 2: $1500 \mathrm{rpm} / \mathrm{s}$ \\
& Time 2: $30 \mathrm{~s}$ \\
\hline Soft Bake & Hotplate $1 \mathrm{~min} / 100^{\circ} \mathrm{C}$ \\
\hline Exposure & $70 \mathrm{~mJ}$ \\
\hline Hard Bake & Hotplate $50 \mathrm{~s} / 105^{\circ} \mathrm{C}$ \\
\hline Development & $300 \mathrm{MIF}$ Full Concentration, $60-120 \mathrm{~s}$ \\
\hline Descum & $\mathrm{O}_{2}$ Plasma Etch for $60 \mathrm{~s}$ \\
\hline
\end{tabular}

\section{AZ 4620 (Positive Tone)}

Table 3-5: AZ 4620 Recipe

\begin{tabular}{|l|l|}
\hline Dehydration Bake & Hotplate $5 \mathrm{~min} / 120^{\circ} \mathrm{C}$ \\
\hline Spin Coat & Resist Thickness: 7um \\
& Spin 1: $1790 \mathrm{rpm}$ \\
& Ramp 1: $550 \mathrm{rpm} / \mathrm{s}$ \\
& Time 1: $9 \mathrm{~s}$ \\
& Spin 2: $3000 \mathrm{rpm}$ \\
& Ramp 2: $5000 \mathrm{rpm} / \mathrm{s}$ \\
& Time 2: $60 \mathrm{~s}$ \\
& Spin 3: $7000 \mathrm{rpm}$ \\
& Ramp 2: $5000 \mathrm{rpm} / \mathrm{s}$ \\
& Time 2: $10 \mathrm{~s}$ \\
\hline Soft Bake & Oven 25min/110 ${ }^{\circ} \mathrm{C}$ \\
\hline Exposure & $350 \mathrm{~mJ}$ \\
\hline Hard Bake & N/A \\
\hline Development & AZ400K $3($ DI-Water):1(Developer), \\
& $180 \mathrm{~s}$ \\
\hline Descum & $\mathrm{O}_{2}$ Plasma Etch for 60s \\
\hline
\end{tabular}




\section{AZ 5214 IR (Positive Tone)}

Table 3-6: AZ 5214 Positive Tone Recipe

\begin{tabular}{|l|l|}
\hline Dehydration Bake & Hotplate $5 \mathrm{~min} / 120^{\circ} \mathrm{C}$ \\
\hline Spin Coat & Resist Thickness: $1.2 \mathrm{um}$ \\
& Spin 1: $500 \mathrm{rpm}$ \\
& Ramp 1: $250 \mathrm{rpm} / \mathrm{s}$ \\
& Time 1: $5 \mathrm{~s}$ \\
& Spin 2: $5000 \mathrm{rpm}$ \\
& Ramp 2: $2500 \mathrm{rpm} / \mathrm{s}$ \\
& Time 2: $30 \mathrm{~s}$ \\
\hline Soft Bake & Hotplate $1 \mathrm{~min} / 110^{\circ} \mathrm{C}$ \\
\hline Exposure & $230 \mathrm{~mJ}$ \\
\hline Hard Bake & N/A \\
\hline Development & $300 \mathrm{MIF}$ Full Concentration, $60 \mathrm{~s}$ \\
\hline Descum & $\mathrm{O}_{2}$ Plasma Etch for $60 \mathrm{~s}$ \\
\hline
\end{tabular}

All these resists were used at some point in the making and characterization of the MTJs. Towards the end, AZ 5214-IR proved to be the most convenient given its dual-tone capability. Furthermore, it is also the thinnest resist allowing for higher resolution features. If aggressive etching was needed, AZ 4620 is the thickest photoresist available with an overcut profile.

\subsection{Etching}

Lithography allows for both additive and subtractive processes. Film deposition by physical and chemical vapor deposition techniques are examples of additive process. In this section, etching will be discussed where layers of film are taken away rather than added. A masking material must be chosen appropriately to ensure proper selectivity. Selectivity $(\boldsymbol{S})$ is the ratio of the etch rate of the film $\left(\boldsymbol{E}_{f}\right)$ and the masking material $\left(\boldsymbol{E}_{\boldsymbol{m}}\right)$ $\left(S=\frac{E_{f}}{E_{m}}\right)$. Poor selectivity will result in etching unwanted areas. Etching goes through 
three processes: The first is the transport of the reactants on to the surface of the target film; the second is the actual reaction between the film and the reactants; and third is the transport of the reaction products away from the surface of the target film. These processes are important to understand and control for keeping a consistent and uniform etch rate. During the etch process, the patterned are can also be undercut, creating two etch profiles: Isotropic and Anisotropic. The amount of undercut is known as the etching "bias." An isotropic etchant erodes the material equally in all directions producing round sidewalls and resulting in large etch bias. Anisotropic etching will occur when there is a preferential direction of erosion creating sharp, vertical sidewalls and a low etch bias. The level of anisotropy $\left(\boldsymbol{R}_{\boldsymbol{H} / V}\right)$ can be measured by the ratio of the horizontal etch rate $\left(\boldsymbol{R}_{\boldsymbol{H}}\right)$ and the vertical etch rate $\left(\boldsymbol{R}_{V}\right)\left(R_{H / V}=\frac{R_{H}}{R_{V}}\right)$. Anisotropic etching is preferred in the devices developed in this thesis for the feature sizes to remain intact.

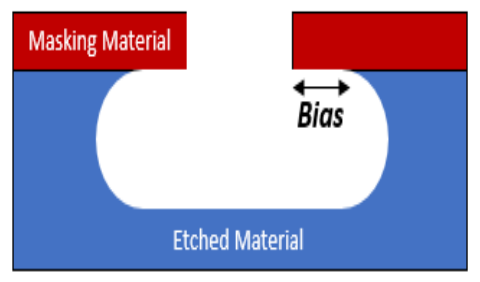

Isotropic: $R_{H N}=1$

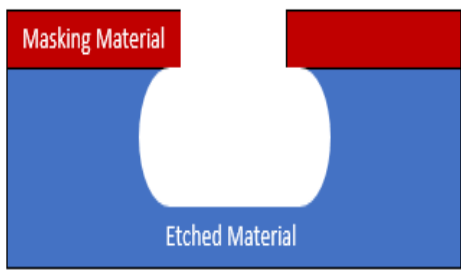

Anisotropic: $0<R_{H N}<1$

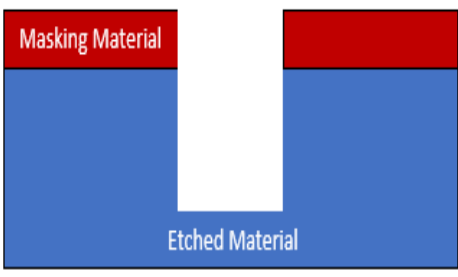

Completely Anisotropic: $R_{H N}=0$

Figure 3-18: Etch Profiles

Etching can be broken down into two classes: wet and dry. Wet etching involves submersing wafers in liquid etchant solution. This method is cheap and simple, but difficult to control and replicate. Most wet processes are highly selective, have fast etch rates, and high throughput. They also tend to be isotropic, except for crystalline materials where the etch rate is lower on the more densely packed direction (ex: Si etches 100 times faster in the [100] plane than the [111] plane). The etch rate uniformity is 
controlled by consistently transporting new reactants to the surface of the target material and removing reaction products by stirring. The etch rate can also be increased by temperature, a process that's easy to do in liquids. Wet etching is a popular method for many microfabrication processes (ex: bulk silicon etching), but at the nanoscale it becomes difficult to apply because of its large etch bias. Wet etching was mostly used in this thesis to make chromium based masks for photolithography, substrate preparation, and some miscellaneous etch processes attempted for developing MTJs.

Dry etch processes include plasma-assisted chemical reaction using gas etchants, physical sputtering, and reactive ion etching. Plasma etching involves the use of an etch species (responsible for the plasma) such as neutral atoms or radicals. The plasma creates chemical reactions with the surface of the sample at room temperature that would otherwise require high temperatures. Radicals combine with the target material forming volatile byproducts. Plasma etching is a low damage isotropic etch process with good selectivity. Physical sputtering involves bombarding ions onto the substrate, mechanically ejecting the material. It is a physical process with high directionality and is therefore anisotropic. The etch rate tends to be low, have poor selectivity, and issues with re-deposition. Reactive ion etching is a physical and chemical combination. It combines both ions and neutral radicals, where the ion bombardment promotes enhanced reaction and etching directionality. Furthermore, it breaks up any byproducts that remain on the surface of the substrate that may inhibit the reaction process. This process has fairly good selectivity and remains rather anisotropic. Dry methods are heavily influenced by the pressure, energy, and gas species. At higher pressures and lower energies, the process is more chemical than physical. If a process is more physical, it tends to be more 
anisotropic with less selectivity, while the opposite is true for a more chemical process. Plasma etching and reactive ion etching were used frequently for photo-stripping, descumming, and some material etch processes that were attempted in fabricating the MTJs.

\subsubsection{Focused Ion Beam}

An important etch tool in the development of MTJs was the focused-ion-beam (FIB). It falls under the category of dry etching, and it is primarily a physical, milling process. FIB works in a similar fashion as a scanning electron microscope, but instead of a focused beam of electrons to image a sample, it uses a focused beam of ions that can be used for both imaging and milling. This tool has recently been used in the semiconductor industry to fix or modify semiconductor devices by milling or filling vias, making connections and disconnection lines, prepare samples for transmission electron microscopy (TEM), and develop prototypes. The ions greater mass allows atoms to be easily ejected from the surface of samples and produce secondary electrons, hence the milling and imaging capability. The milling capability allows for micro- and nanofabrication etch processes for a large variety of materials with nanometer scale precision. Furthermore, the secondary electron imaging reveals intense grain orientation contrast and with enough skill from the user, even grain boundary contrast.

The ion beam consists of metal ions generated from a liquid metal ion source (LMIS). Of the many metallic elements and alloys used, Gallium $\left(\mathrm{Ga}^{+}\right)$is the most preferred because of its mechanical, electrical, and vacuum properties. Gallium also has a low melting point $\left(\sim 30^{\circ} \mathrm{C}\right)$, which minimizes any diffusion between the liquid and the tungsten needle that emits the ions. It has low volatility and low vapor pressure at the melting point, 
increasing its shelf-life. High angular intensity with a small energy spread is possible because of its emission characteristics. Once emitted from the LMIS, the ions are accelerated and focused on the sample using electrostatic lenses. A condenser lens is used to form the probe and an objective lens to focus the beam at the sample. Beams generated can be anywhere between a couple picoamperes to 50nA, depending on whether you want to image or mill a sample.

The most common use of FIB in the devices made in this thesis was the milling application. It is a sputtering process where atomic collisions between the metal ions and the surface result in the removal of material on the surface of the substrate. The amount removed depends heavily on the beam current and the accelerating voltage. The advantage of this tool is the capability to etch without requiring any patterning techniques. Milling and imaging do have certain disadvantages which are related to the current beam and voltage parameters. During the FIB process, the films are at risk for ion implantation which can alter the material properties (thermal, electric, etc.) and physical structure. Re-deposition of the milled material is also a problem, creating defects in the devices. Careful choice of FIB parameters are characterized to ensure that the quality of the devices remain intact. Characterization involving film penetration versus beam strength was done. Samples for high resolution TEM were also prepared with FIB to analyze the cross-section of the MTJs fabricated in this thesis. 


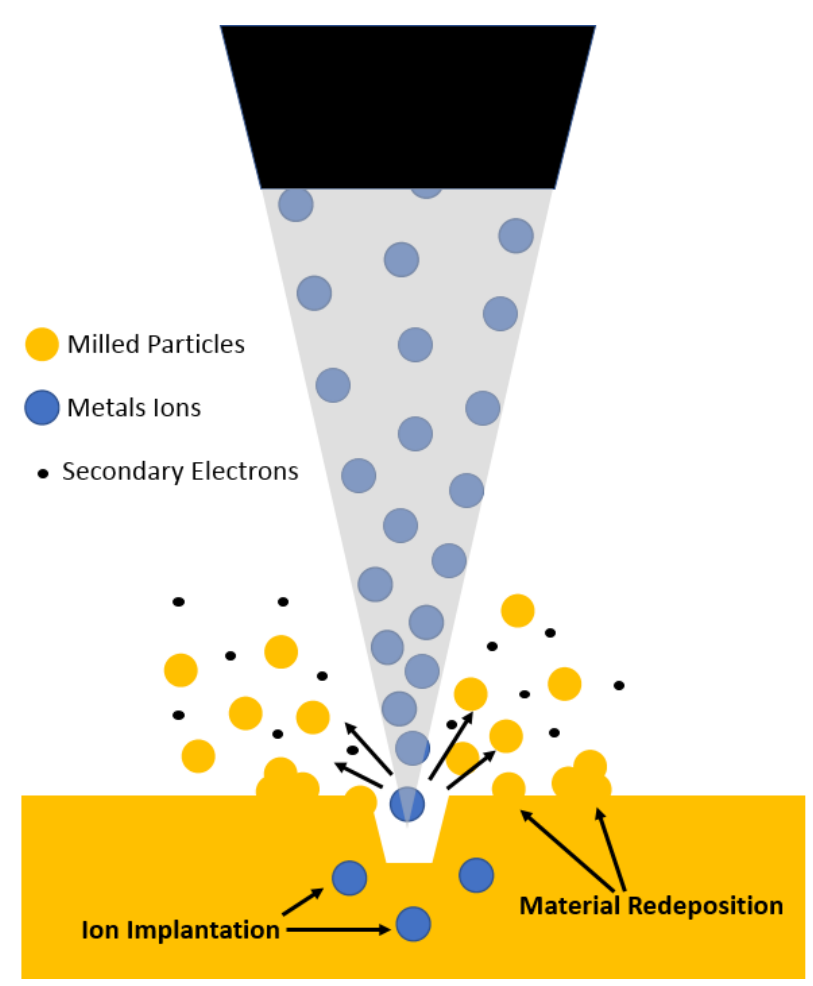

Figure 3-19: Focused Ion Beam Schematic

The FIB tool used was the JIB-4610F, which in addition to FIB column it also has an SEM microscope for high resolution analysis. The FIB column provides a maximum ion current of $90 \mathrm{nA}$ at $30 \mathrm{kV}$ with a resolution capability of $\sim 5 \mathrm{~nm}$. The SEM has a maximum probe current of 200nA and has high resolution analytical tools like energy dispersive $\mathrm{x}$ ray spectroscopy (EDS) and electron back scatter diffraction (EBSD). This setup is known as a dual-beam system where the advantages of both SEM and FIB complement each other and are available in a single tool. The typical dual-beam column setup is having a vertical electron column with a tilted ion column. Electron beams are nondestructive and offer some unique analytical advantages that ion beams cannot. During the FIB process, the electron beam was used to monitor the ion-beam milling. 


\subsection{Particle Synthesis}

Nanoparticles are typically range between 1 and 100nm in size. As mentioned previously, this intermediate size range between atoms and bulk material exhibit unique properties that can be exploited. Nanoparticles have found utility in a variety of fields such as medical treatment, magnetic recording media, solar cells, biosensors, cosmetics, etc. Chemical, physical, and biological processes are used for making nanoparticles [47]. Chemical processes include: Sol-gel method, chemical precipitation, hydrothermal synthesis, etc. Physical methods are for the most part a top down method that involves applying mechanical pressure, high energy radiation, thermal, and electric energy. Typical processes include: milling, laser ablation, inert gas condensation, and physical vapor deposition. Synthesis involving biology makes use of microorganisms, enzymes, plant extracts, etc. Bio-assisted processes have the unique advantage of being environmentally friendly and cost effective. Given the magnitude of this field, this section will focus specifically on the synthesis of cobalt ferrite $\left(\mathrm{CoFe}_{2} \mathrm{O}_{4}\right)$ nanoparticles, since they are the ones used in this thesis.

Co-precipitation is the most common and cost-effective method used to make ferrite nanoparticles. This process involves soluble substances being carried out of a solution by a precipitate. Despite usually being undesired in chemistry, it is an occurrence that is exploited. Co-precipitation can be caused by an inclusion, where an impurity is embedded in the crystal structure of the carrier. An impurity can also be weakly bound to the surface of the precipitate by a mechanism called adsorption. The impurity could also be physically trapped in the precipitate, an occlusion. It is an inexpensive chemical method where the size of the particle depends on the level of control of the relative rates 
of nucleation and growth during the synthesis process. This includes monitoring the reaction temperature, stir rate, $\mathrm{pH}$ value, and ionic strength of the media.

During the synthesis of the cobalt ferrite nanoparticles, the reaction was done in air, a medium that is known to provide good yield. It is also the simplest environment since an apparatus or chamber isn't needed to house the reaction. Characterization of the process was carefully done and it was found that there is a strong relation between reaction temperature and particle size. During the chemical process, nuclei are formed. These nuclei are responsible for the crystal formation and result in the creation of interfaces between the solid nuclei and the liquid solution. Initially, the nuclei exhibit high interfacial area that increases the free energy of the system. This energy is mitigated by the nuclei increasing in volume, resulting in particle growth. Consequently, bringing the reaction temperature down in turn brought the free energy down, resulting in smaller particles. Controlling aggregation (the clustering of particles) is another concern. Nanoparticle motion is governed by Brownian mechanics, where collisions can cause attraction due to Van-der-Waals and magnetic forces. Increasing the $\mathrm{pH}$ of the solution and the ionic strength influences surface charges on the particles, resulting in repulsion. In a further attempt to keep the nanoparticles from aggregating, the particles are eventually coated with a surfactant, such as oleic acid. The surfactant also prevents the particles from oxidizing. Once particles are synthesized, their size is verified with AFM, and magnetic properties are observed with magnetic force microscopy (MFM) and an alternating gradient magnetometer (AGM). 
The following recipe has been characterized for making $2 \mathrm{~nm}, 4 \mathrm{~nm}$, and $10 \mathrm{~nm}$ cobalt ferrite nanoparticles:

1. Prepare the following 3 solutions:

- Solution a: Cobalt nitrate hexahydrate: $0.98 \mathrm{~g}$ in $50 \mathrm{ml}$ DI water

- Solution b: FeCl2: $1.27 \mathrm{~g}$ in 50ml DI water

- Solution c: $\mathrm{NaOH}: 2.8 \mathrm{~g}$ in $20 \mathrm{ml}$ DI water

2. Solutions a and $b$ are mixed. For different size particles, the mixture is heated at:

- $\mathrm{T}=40^{\circ} \mathrm{C}$ for $2 \mathrm{~nm}$ particle 0073

- $\mathrm{T}=60^{\circ} \mathrm{C}$ for $4-6 \mathrm{~nm}$ particles

- $\mathrm{T}=80^{\circ} \mathrm{C}$ for $10-15 \mathrm{~nm}$ particles

3. Once heated, solution $\mathrm{c}$ is added and stirred for 30 minutes

4. Add $1 \mathrm{ml}$ of Oleic Acid and continue stirring for 2 hours

5. Remove heat and let the solution cool naturally

6. Rinse nanoparticles several times with DI-Water or ethanol

7. Dry nanoparticles in a vacuum chamber 


\section{TECHNOLOGICAL APPLICATIONS}

\subsection{Introduction}

Magnetics has dominated the memory storage industry thanks to the discovery of the GMR effect. These magnetic effects led to the development and commercialization of hard disk drives (HDD). This field has been further augmented with the discovery of the TMR effect, expanding the theoretical capability of magnetics to move beyond memory storage and onto information processing (MRAM). Magnetic based information processing yet remains a niche role and cannot dominate the market unless the switching energy is brought down substantially [48].

A magnetic tunnel junction (MTJ) is simply defined as two ferromagnetic electrodes separated by an ultra-thin oxide. Tunneling is strictly a quantum mechanical effect where a particle tunnels through a barrier which would otherwise be impossible to transverse according to classical mechanics. Due to the wave-particle duality of electrons, conduction through a thin, insulating barrier is possible with a certain degree of probability. The fermi levels of the two electrodes separated by the insulator reside in the bandgap of the tunneling barrier. For the sake of simplicity, we can picture a rectangular potential barrier formed, where the height depends on the tunneling barrier conduction band. With the absence of a voltage, the two fermi levels from the electrodes are aligned and the system is in thermodynamic equilibrium, hence no current conducts. Applying a voltage changes the barrier shape and separates the two fermi levels by an amount $\mathrm{eV}$ (e being the charge of an electron and $\mathbf{V}$ the applied voltage). Conduction becomes possible and depends heavily on the density of states of the electrodes and the tunneling probability. 
The probability of conductance depends on the relative magnetic orientation of the two ferromagnetic layers. Initially Jullière experimentally demonstrated the TMR effect using Germanium oxide as the tunneling barrier. The effect was small and found at absolute temperatures $(\sim 0 \mathrm{~K})$. Jullière provided the first interpretation of TMR and assumed that electron spin is conserved during the tunneling process and depended on the spin polarization of the ferromagnetic electrodes $\left(\frac{2 P_{1} P_{2}}{1-P_{1} P_{2}}\right)$. While many oxides were experimented with, most failed to show substantial TMR. Amorphous $\mathrm{AlO}_{\mathrm{x}}$ barriers emerged and were experimentally proven to provide significant TMR $(\sim 15 \%$ at room temperature) $[49,50]$. Improving the quality of the barrier oxide and combining it with ferromagnetic electrodes with high polarization, a TMR of up to 70\% was achieved [51]. Eventually, crystalline $\mathrm{MgO}$ was introduced as a tunnel barrier with a much higher theoretical TMR than $\mathrm{AlO}_{\mathrm{x}}$ [52]. However, to achieve high TMR, $\mathrm{MgO}$ must be crystalline with a [001] texture. Furthermore, the $\mathrm{MgO}$ tunnel barrier must interface coherently with the [001] plane of body-centered-cubic (BCC) ferromagnetic electrodes. A TMR of $400 \%$ has been reported with $\mathrm{Co} / \mathrm{MgO} / \mathrm{Co}$ junctions. In addition to the increase in TMR by an order of magnitude, $\mathrm{MgO}$ films could easily be deposited with magnetron sputtering, an industry compatible film deposition process.

The reason amorphous $\mathrm{AlO}_{\mathrm{x}}$ was chosen as a tunnel barrier was due to its ability to form thin, dense, and smooth barriers. Interface coherency, however, can dramatically improve TMR since tunneling becomes more spin dependent and is subject to less scattering [53]. Coherent tunneling results in a spin-dependent match within the evanescent states in the 
tunneling barrier and the electronic states of the interfacing ferromagnetic layers. Because $\mathrm{AlO}_{\mathrm{x}}$ tunneling barrier is amorphous, there is no crystallographic symmetry. All the Bloch states from the electrode can therefore couple with the evanescent states of the tunneling barrier (incoherent tunneling). Different Bloch states with different spin polarizations will tunnel, resulting in a net spin polarization. When the ferromagnet/oxide is properly interfaced, only select Bloch states from the electrode couple with the evanescent states of the tunneling barrier. With coherent tunneling, a spin filtering effect can be established, allowing for theoretically high TMR ratios. While the Jullière model successfully describes incoherent tunneling that occurs in amorphous $\mathrm{AlO}_{\mathrm{x}}$ barriers, it is unable to properly describe TMR for crystalline $\mathrm{MgO}$. Slonczewski proposes a model that addresses additional factors beyond the simplified version proposed by Jullière.

$\mathrm{MgO}$ based MTJs required lattice matching and correct crystalline orientation of the ferromagnetic films to achieve high TMR. Only a handful of ferromagnetic electrodes meet this criterion: $\mathrm{Fe}, \mathrm{CoFe}, \mathrm{Co}$ (BCC crystal orientation), and $\mathrm{CoFeB}$ [54]. Fabricating these MTJs is challenging because it's hard to control crystallinity in most physical vapor deposition techniques. Furthermore, the MTJ films must be smooth and uniform to avoid further complications such as hot spots and orange-peel coupling. This issue was solved by using $\mathrm{CoFeB}$ as the material of choice [55]. $\mathrm{CoFeB}$ when sputtered is amorphous but smooth. Boron is responsible for the amorphous nature of the film, which results in reduced interface roughness. When $\mathrm{MgO}$ is sputtered on top, it naturally forms a welloriented [001] texture [56]. A post-annealing process at around 350 degrees Celsius will promote crystallization of $\mathrm{CoFeB}$, which forms epitaxially between the two $\mathrm{MgO} / \mathrm{CoFeB}$ interfaces. A BCC crystallized structure will form with a well-matched lattice, where 
$\mathrm{MgO}$ acts like a template for the crystallization growth. A TMR of up to $600 \%$ at room temperature has been reported with $\mathrm{CoFeB}(001) / \mathrm{MgO}(001) / \mathrm{CoFeB}(001)$ junctions. This structure is configured to enable a coherent tunneling process, resulting in a high TMR $[57,58]$.

\subsection{FIB-MTJ}

\section{Theory}

Spin transfer torque based magnetic tunnel junctions have the potential to become a universal memory. To become a universal memory, it must compete with other technologies in terms of latency (SRAM), packing density (DRAM), and non-volatility (FLASH) [11-13]. STT-MTJs can perform non-volatile logic with near zero static power consumption. The use of current instead of external magnetic fields allows for a higher density of magnetic elements since they will no longer be prone to accidental writing. However, increasing the density of storage elements is an on-going challenge [59]. We must scale MTJs down in size to achieve higher density. This poses a challenge in both fabrication (lithography limitations) and performance (thermal stability). For ferromagnetic films that are magnetized in-plane, shape anisotropy is responsible for keeping the magnetization stable. As the size of the MTJ goes down, shape anisotropy is unable to keep up with thermal fluctuations. Longitudinal recording below a critical dimension becomes superparamagnetic [60]. However, materials that exhibit perpendicular magnetic anisotropy (PMA) provide more thermal stability in part due to the significantly reduced demagnetization fields [61]. For PMA materials, magnetization points out of plane rather than in-plane. Furthermore, perpendicular MTJs have experimentally shown to have a more efficient spin-transfer torque switching mechanism 
than those that are in-plane (an order of magnitude), resulting in a decrease in the amount of switching current. These fundamental limitations only allow longitudinal recording to achieve $100 \mathrm{Gbit} / \mathrm{in}^{2}$ density [62], while perpendicular recording can push the limit to greater than $1 \mathrm{Tbit} / \mathrm{in}^{2}[63]$.

MTJs with thin $\mathrm{CoFeB}$ ferromagnetic films and $\mathrm{MgO}$ as the tunnel barrier exhibit strong PMA due to the anisotropy originating from the interface. However, if the CoFeB films aren't thin enough, shape anisotropy will dominate and they will be magnetized in-plane [64]. Careful characterization of the film is needed because making it too thin brings the energy barrier down. In this thesis, a pseudo spin valve (PSV) configuration is used: $\mathrm{Ta}(5$ $\mathrm{nm}) / \mathrm{CoFeB}(1 \mathrm{~nm}) / \mathrm{MgO}(0.9 \mathrm{~nm}) / \mathrm{CoFeB}(1 \mathrm{~nm}) / \mathrm{Ta}(5 \mathrm{~nm})$ [34]. Ta acts as a seed layer to promote exchange coupled films. In addition to promoting PMA, Ta also serves as a protective layer for the MTJ. This film composition is capable of withstanding annealing temperatures for long periods of time, a necessary step to ensure crystallinity between $\mathrm{CoFeB}$ and $\mathrm{MgO}$. The expected coercivity of the free layer should be $\sim 50$ Oe and the fixed layer $\sim 100$ Oe. This type of multi-stack configuration avoids the use of an antiferromagnet to pin one of the ferromagnetic layers. Studies have shown that higher TMR can be obtained with PSV than with exchange biased spin valves. Overall, this MTJ configuration provides ultra-high perpendicular magnetic anisotropy energy (necessary for thermal stability), high spin torque efficient (improved STT effect), and high magnetoresistance (to ensure adequate SNR).

The main challenge in making this technology dominant in the information processing industry is addressing the power required to switch the magnetic orientations of the ferromagnetic films in the MTJ. Moore's law is nearing and while semiconductors begin 
to have problems when they are scaled down too small, we find that in the sub-10nm range, MTJs begin to improve in performance [9]. Currently, as we make MTJs smaller, the switching current scales linearly with size. MTJs as small as $20 \mathrm{~nm}$ have been reported with switching current densities as low as $\sim 3.9 \mathrm{MA} / \mathrm{cm}^{2}$ [65]. However, below 10nm is an intermediate size range that isn't well understood in the field of spintronics. A continuous crystalline lattice model would no longer apply since what we have is more of a cluster of atoms where energy exchange between excitations becomes less effective. This should reduce any damping factors and significantly promote more efficient magnetic switching. Ab-initio calculations that considered complex atomistic-scale quantum theory simulated coherency of spins with respect to size $[10,66]$. Two size ranges of interest were modeled, those above and below 10nm. When a reverse magnetic field is applied to the magnet it triggers a relaxation process. For sizes greater than $10 \mathrm{~nm}$, there is clear de-coherence and therefore a very short relaxation time. Those modeled below $10 \mathrm{~nm}$, the coherency between spins is not immediately broken and there is negligible damping, resulting in a much longer relaxation period.

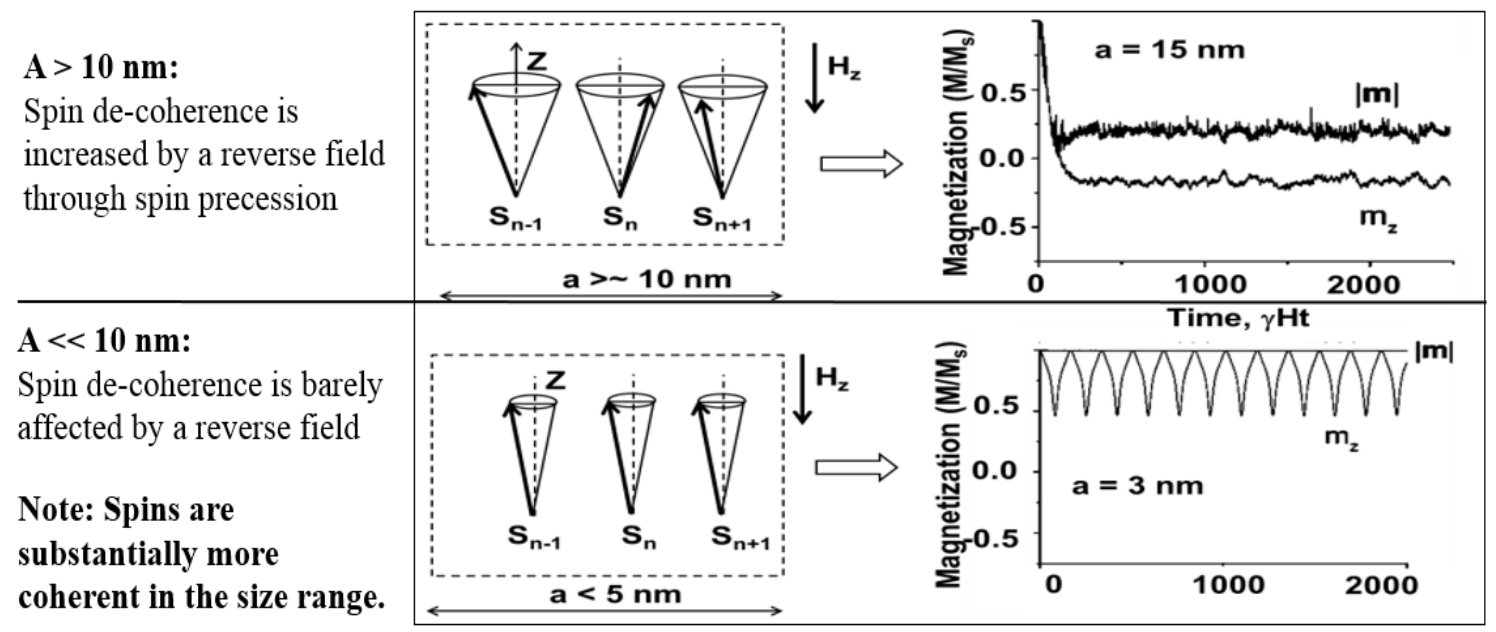

Figure 4-1:Ab Initio Quantum Mechanical Calculations [66] 
In the sub-10nm region thermal reservoirs, that are usually responsible for absorbing spin excitations, become extremely small [10]. This means that they are unable to absorb the energy from the different spin dynamics in play. This intermediate size breaks away from the continuous crystal lattice models that tend to dampen magnetization. A reduced damping will increase the efficiency, and thus the energy for switching. The MTJ device used in this research has a film composition that has already been proven to provide a high TMR, high anisotropy, and small switching current densities. The object of this research is not to optimize the film parameters to improve the performance, but rather monitor the change in performance as we scale them down to the nanoscale level.

The switching current density can be expressed with Slonczewski's phenomenological expression [67]: $J_{C O} \approx M_{S} \frac{\alpha t}{p \xi}\left(H_{k} \pm H_{0}\right)$, where $\boldsymbol{H}_{\boldsymbol{K}}$ is the anisotropy field, $\boldsymbol{H}_{\boldsymbol{O}}$ is the anisotropy field, $\boldsymbol{M}_{S}$ is the saturation magnetization, $\boldsymbol{t}$ is the thickness of the free layer, $\boldsymbol{p}$ is the spin polarization, $\boldsymbol{\xi}$ is the spin torque efficiency factor, and $\boldsymbol{\alpha}$ is the damping constant. For large devices (greater than 10nm), there is a coupling to phonons due to the elastic modulation of exchange interactions and the crystalline fields. L-S interactions are an important factor in spin-lattice relaxation. Spin-lattice relaxation is the mechanism where the magnetization vector reaches thermal equilibrium with respect to the lattice. Spin-lattice relaxation and thermal agitations will hinder the life-span of a signal. Below $10 \mathrm{~nm}$ we can expect much longer spin life, which would make the switching dynamics more efficient. We can model the spin relaxation as a function of surface versus volume:

$$
\tau_{S} \sim d /\left[\left(1-N_{S} / N_{V}\right) \Delta g_{V}^{2}+\left(N_{S} / N_{V}\right) \Delta g_{S}^{2}\right] V_{F}
$$


The parameter $\boldsymbol{d}$ is the diameter of the MTJ, $\Delta \boldsymbol{g}$ is the $\mathrm{g}$-factor shifts for both volume and surface, $\boldsymbol{N}$ is the electron concentration for both volume and surface. For larger structures, the g-factor shift at the surface is negligible compared to the volume. As we decrease the size, the surface begins to dominate and as the surface vs. volume ratio increases, so does the relaxation time. The g-factor shift at the surface is typically orders of magnitude less than in the volume, so below $5 \mathrm{~nm}$ we can expect to see the spin relaxation time increase by orders of magnitude.

According to basic magnetic theory, scaling at such a small size comes at the cost of thermal stability $\left(\Delta=\frac{K V}{k T}\right)$, where the MTJ would become increasingly volatile. We compensated this effect of diminishing volume by increasing the anisotropy with the right material choice and MTJ configuration (PSV film composition). However, as we scale the MTJ down, the scattering effects of temperature should also diminish, therefore indirectly increasing the thermal stability. This effect is yet to be confirmed experimentally.

What initially propelled the experimental efforts to test this theory was the clever idea of fabricating an MTJ on the tip of a nanoprobe. A magnetic tunnel junction was sputtered directly onto the tip of a probe. The tip was sharpened to a point less than $10 \mathrm{~nm}$ in diameter. The probe was then brought into contact with a copper substrate for IV measurements. A contact resistance was $\sim 185 \mathrm{kOhms}$, a magnetoresistance of $29 \%$, and a switching current of 95nA. With respect to the size of the junction, the switching current density is $\sim 0.1 \mathrm{MA} / \mathrm{cm}^{2}$ [66], an order of magnitude less than what would have been expected from linear scaling and 30 times smaller than the smallest value reported to date. The switching was confirmed to be attributed to STT because of the magnetic field 
dependence with respect to the switching current (Slonczewski's phenomenological expression). The magnetic field, when applied in favor of the switching direction, reduced the switching current.
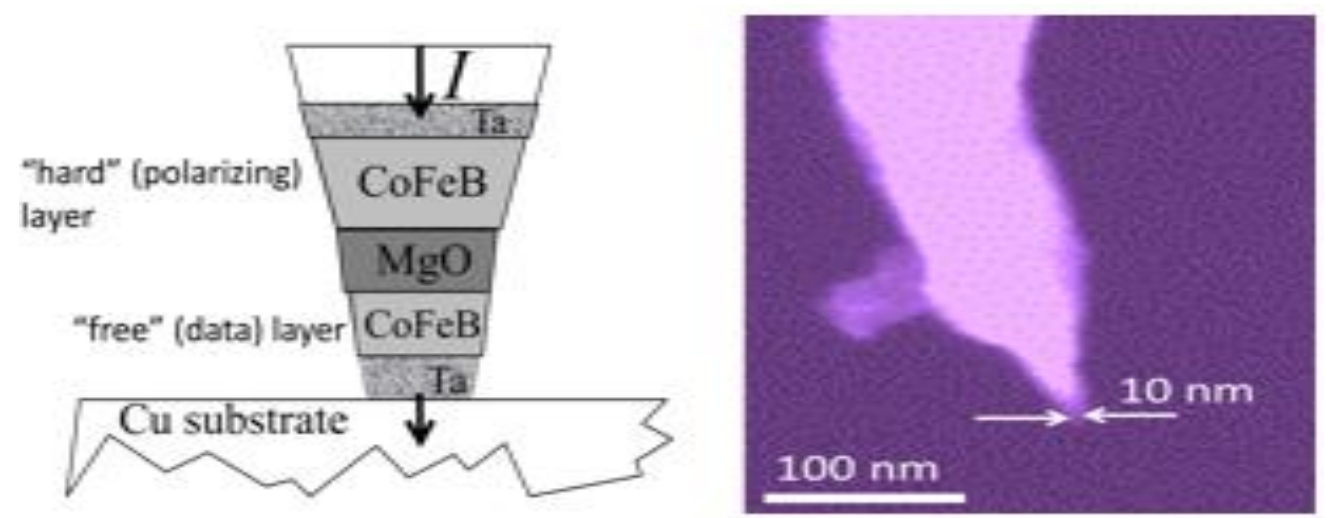

Figure 4-2: Nanoprobe Device Schematic (left) and SEM image of the probe (right) [66]

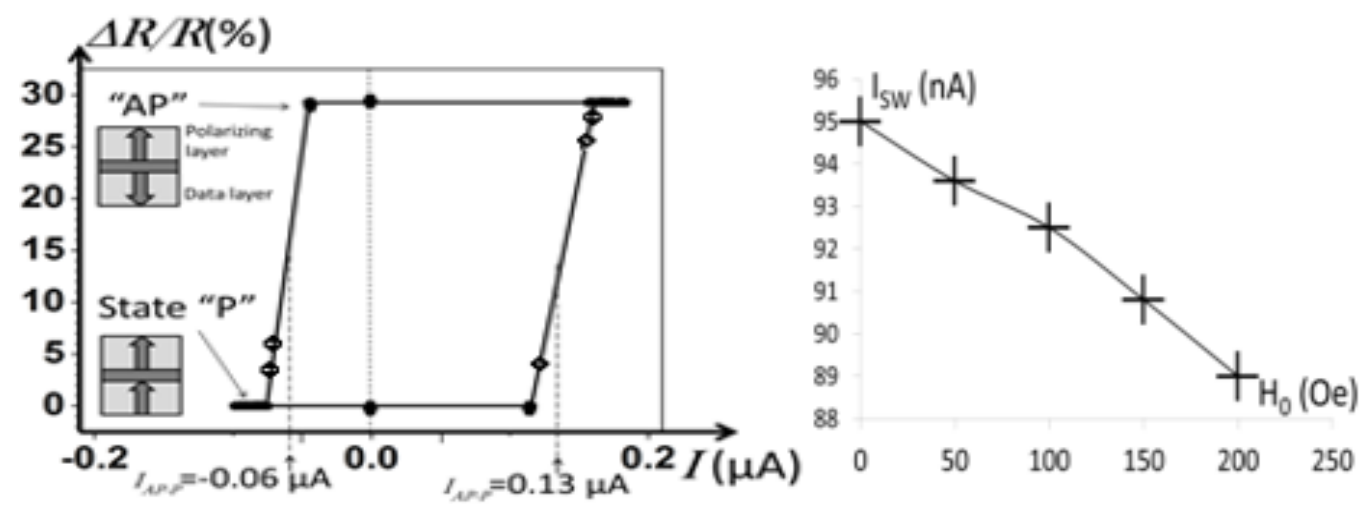

Figure 4-3: TMR Graph (left) and Switching Current vs Field Dependence (right) [66]

This experiment indicates a reduction in the spin damping coefficient by an order of magnitude. The ab-initio calculations modeling magnetic devices below $10 \mathrm{~nm}$ support this outcome. Open questions about this approach include the effect the shape of the probe could have on the magnetic properties, and how the pressure at the point of contact between the device and the copper substrate could impact the measurements. This work was done by Dr. Jeonming Hong and it has been the main motivation in this thesis to further pursue novel methods to develop nanoscale MTJs. 
Because this experiment provided evidence supporting our group's theory with regards to the size dependence of MTJs and the switching current, further work was required to continue to prove our concept. Using a top-down approach, the FIB-based MTJs consisted of making devices using standard photolithographic techniques and bringing them down to the nanoscale using a FIB.

\section{Process Flow}

The MTJ composition consists of a multilayer of films in the PSV configuration mentioned before $(\mathrm{Ta}(5 \mathrm{~nm}) / \mathrm{CoFeB}(1 \mathrm{~nm}) / \mathrm{MgO}(0.9 \mathrm{~nm}) / \mathrm{CoFeB}(1 \mathrm{~nm}) / \mathrm{Ta}(5 \mathrm{~nm}))$. The MTJ is initially patterned in the micrometer range using photolithography and then scaled down to the nanometer level using a focused ion beam (FIB). Once the devices are fabricated, magneto-transport measurements were done. These include measuring magnetoresistance, IV curves, and M-H loops. IV curves and MR runs were done with a lock-in amplifier and Keithley meters. $\mathrm{M}-\mathrm{H}$ loops were done with an Alternating Gradient Magnetometer (AGM) and a Magneto-Optical Kerr Effect microscope (MOKE). Current induced magnetization switching was also used to calculate preliminary TMR to ensure the devices were working properly. Imaging nanoscale devices is challenging given the resolution limits of the SEM. It is more accurate to calculate the size based on the information of the cross section of the device and the electrical resistance. These devices were developed and tested together with Dr. Jeonming Hong, a post-doctoral student in my research group at Florida International University. 


\section{Fabrication Process}

The fabrication process begins with the optical patterning for lift-off of the first contact.

We use e-beam evaporation because of its high throughput and directionality when depositing thin films. We then pattern a small area (5umX5um) with optical lithography and sputter the MTJ films. This is also a lift-off process. This lift-off process must be properly done with sufficient sidewall undercut; otherwise the sputtered films will coat the entire sidewall of the photoresist. As an additional measure to avoid any sidewall shorts, FIB is used to trim around the sidewalls of the device. We again pattern a sidewall around the MTJ, to prevent the top contact from shorting with the sides of the MTJ. Aluminum oxide is used for the sidewall passivation. Finally, we pattern and deposit the top contact using sputtering to ensure ohmic contact.

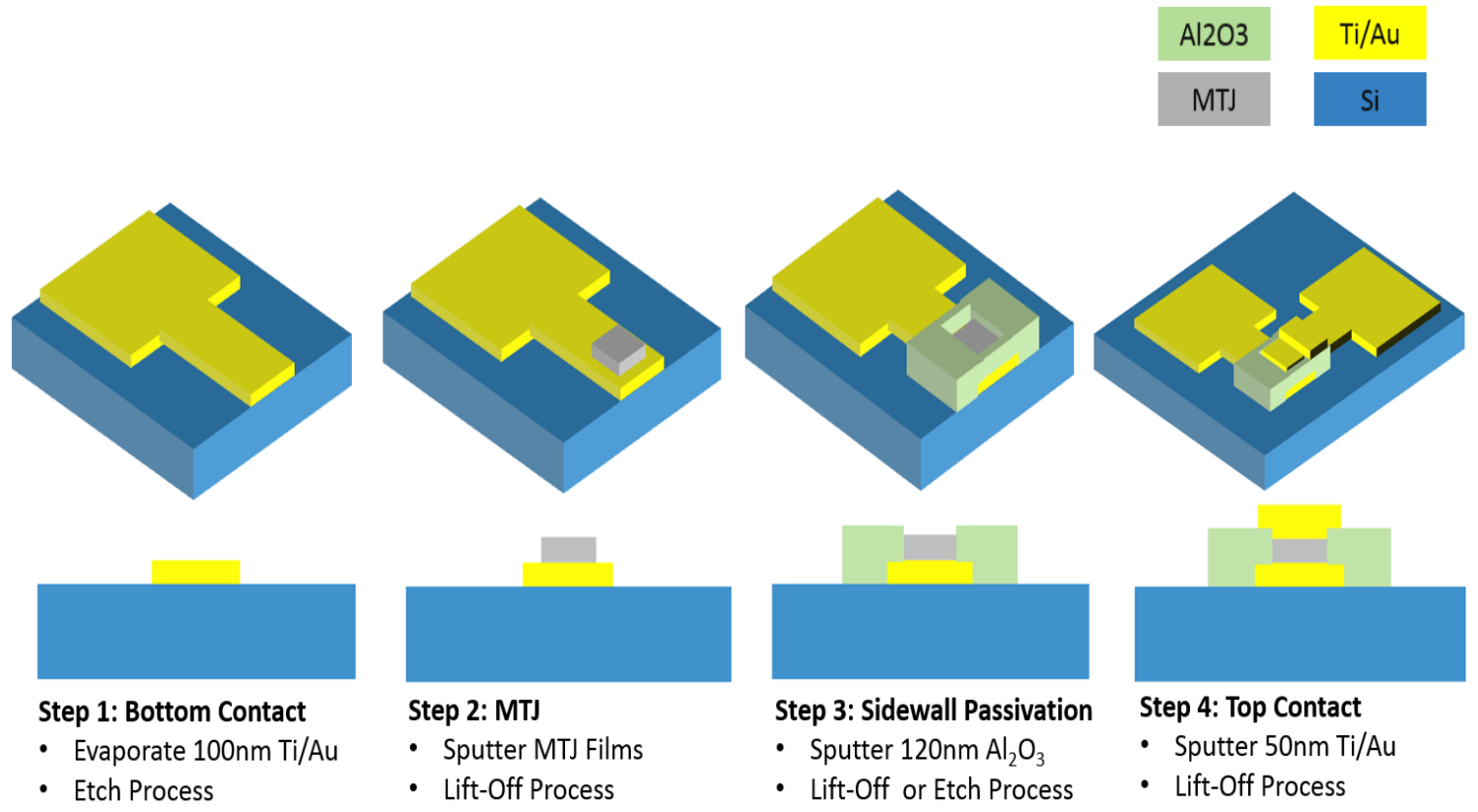

Figure 4-4: Microfabrication Process for developing MTJs 
With the microfabrication process done, we use the Focused Ion Beam (FIB) to trim the devices down to the nanoscale. The overlapping geometry was chosen intentionally so we can easily trim upwards towards a point.
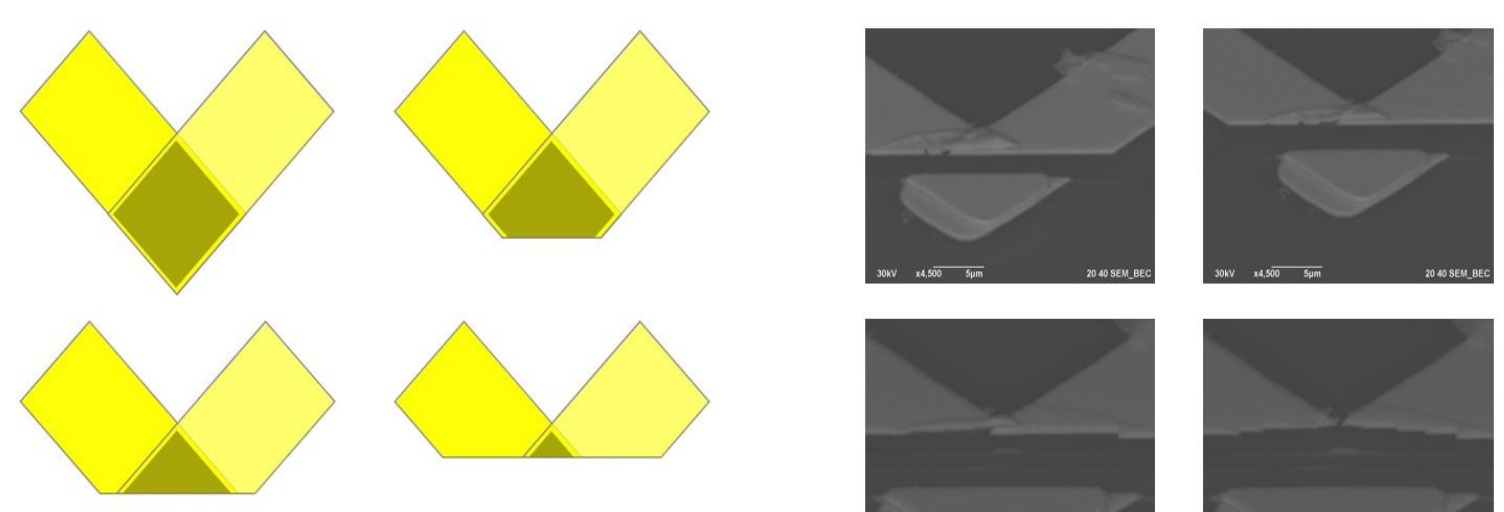

Figure 4-5: Nanofabrication Process for developing MTJs

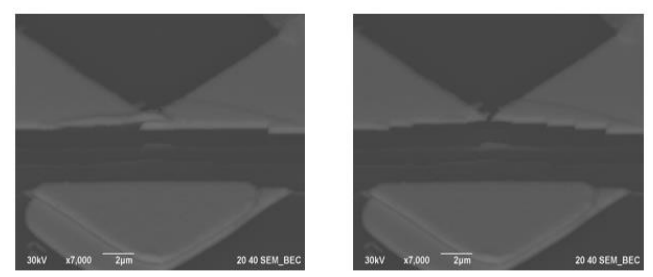

\section{Results}

The smallest device trimmed with the FIB was estimated to be around $5 \mathrm{~nm}$. The measured switching current was around $0.6 \mathrm{uA}$ and a TMR of $60 \%$. The current density is approximately $\sim 1 \mathrm{MA} / \mathrm{cm}^{2}$ [48]. This is comparable to what was measured with the nanoprobe, and is smaller than what would have been predicted by linear scaling. For this size range, as we have mentioned before, the surface effect becomes dominant over the volume which impact the relaxation time. An increase in relaxation time enables a more efficient switching mechanism, bringing the switching current down substantially. While the boundary condition remains somewhat unclear as to when surface effects dominate, we estimate that it is around $10 \mathrm{~nm}$. The thermal stability is estimated to be around $\Delta=$ 20 , which allows the storage layer to be stable for $\sim 1$ second. This time is acceptable for volatile memory applications. Should we increase the anisotropy energy by a factor of two $(\Delta=40)$; the device would hold its memory for 10 years. 


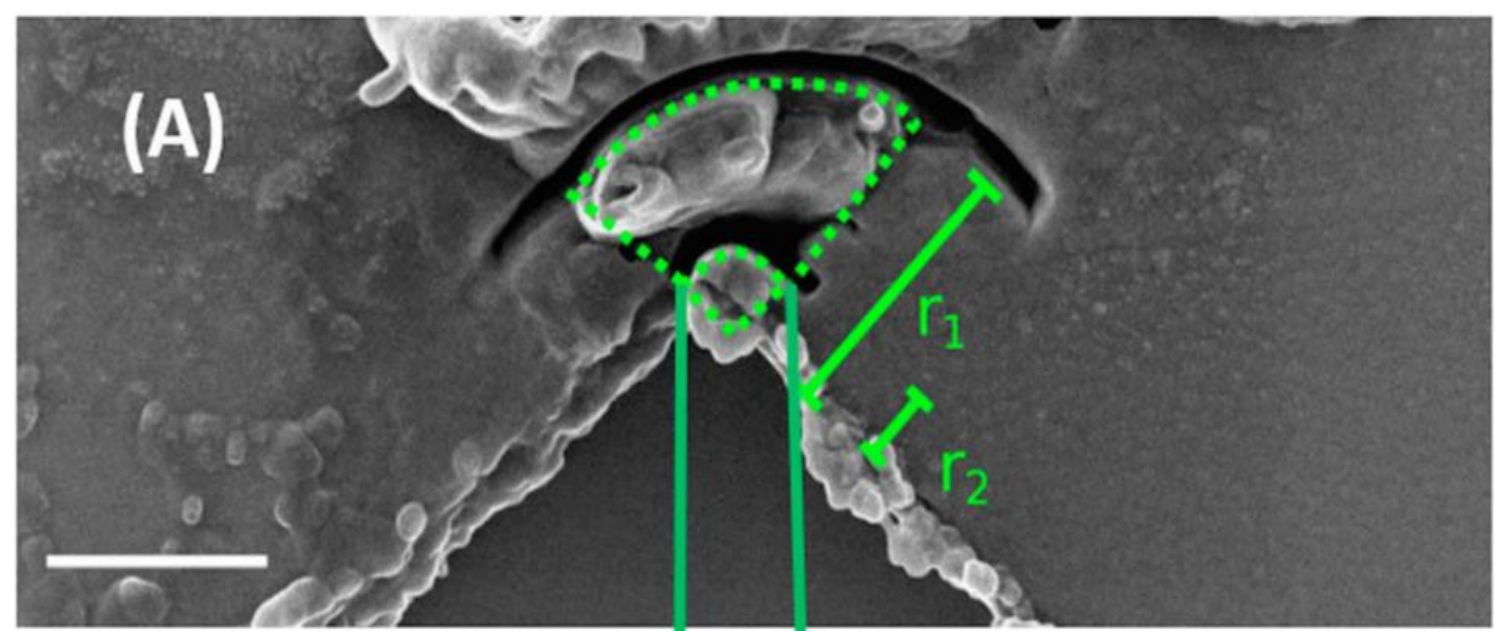

Figure 4-6: FIB Trimmed MTJ [48]
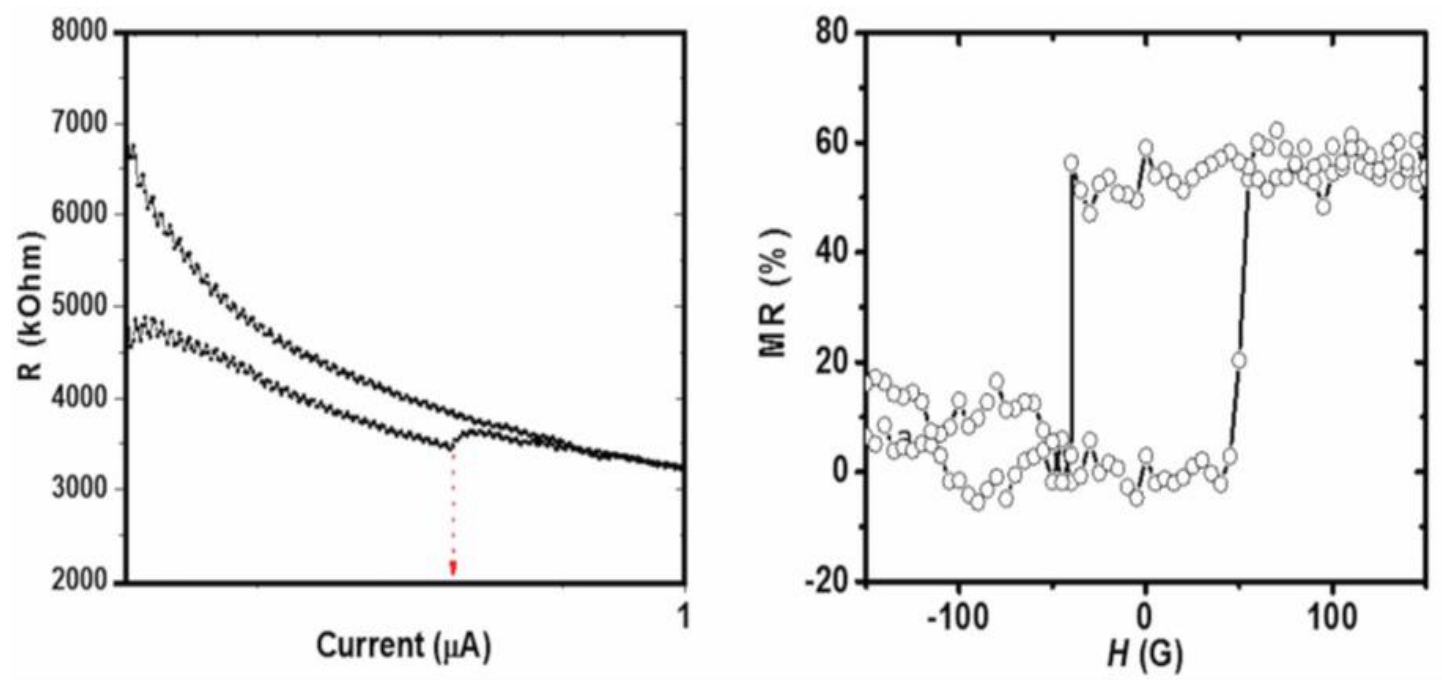

Figure 4-7: IV Measurement (left) and MR Measurement (right) [48]

A key difference between the FIB approach and the probe approach is the susceptibility of Ga implantation in these devices. We are unsure of the impact (if at all) that it could have on the device performance. Despite this, these FIB based MTJs have provided significant evidence supporting the improving behavior of MTJs as we scale them down in the sub-10nm size. 


\subsection{Nanoparticle-MTJ}

So far, several different approaches have been discussed regarding sub-10nm MTJs: Nanoprobe MTJs and FIB assisted MTJs. While we explored the sub-10nm size range, several questions hinder our assessment of the physics in this size regime. These previous methods are difficult to extract exact size dimensions and require difficult fabrication techniques that put a constraint on both resources and time. Another method is discussed in this thesis that involves a bottoms-up approach: nanoparticle based MTJs. In this device we embed 2nm-nanoparticles in a standard MTJ device. At this size range, quantum mechanics dictate the behavior of the device. Due to this size, the thermal reservoir is unable to absorb the magnetic excitation energy. The spin excitation's lifespan increases leading to anomalous magneto-transport effects. Understanding this size regime will allow us to potentially develop future generation spintronic devices.

\section{Theory}

These devices consist of a STT based dual MTJ device, with the main element having a size of $\sim 2 \mathrm{~nm}$. Nanoparticles are sandwiched between two $1 \mathrm{~nm} \mathrm{MgO}$ films which are nestled between two adjacent $1 \mathrm{~nm} \mathrm{CoFeB}$ films. The overall composition is $\mathrm{Ta}(5$ $\mathrm{nm}) / \mathrm{CoFeB}(1 \mathrm{~nm}) / \mathrm{MgO}(1 \mathrm{~nm}) /(2 \mathrm{~nm}$ Nanoparticles $) / \mathrm{MgO}(1 \mathrm{~nm}) / \mathrm{CoFeB}(1 \mathrm{~nm}) / \mathrm{Ta}(5$ $\mathrm{nm})$. This alternate fabrication approach utilizes the nano scale size of the nanoparticles rather than having to use complex top-down methods such as FIB, nano-imprint lithography, electron beam lithography (EBL), etc. There are many well established chemical processes that can synthesize nanoparticles uniformly that tailor to our size criteria and magnetic properties. 
$\mathrm{CoFeO}_{4}$ nanoparticles are ferrimagnetic spinel ferrites that can act as an ideal spin filter in magnetic tunnel junctions. These nanoparticles can behave as half-metals, where they conduct in one spin direction and insulate for the other. Cobalt ferrite is a suitable candidate for spin filtering because it retains its magnetic order above room temperature (Curie temperature is $790 \mathrm{~K}$ ) [68]. The efficiency of a spin filter can be expressed as: $S_{e f f}=\frac{I^{\uparrow}-I^{\downarrow}}{I^{\uparrow}+I^{\downarrow}}$, where $\boldsymbol{I}$ is the current. By integrating nanoparticles into the MTJ we have found evidence of strong half-metal attribute for particles below a certain size. When certain magnetic fields are applied we see steep changes in resistance that could easily be explained by an adjustment of the Fermi level. There is a large and sharp resistance change due the bands near the Fermi level being pushed farther apart as we scale down the size of the nanoparticle. This is in accordance to quantum mechanics where discreetness in energy becomes apparent as the number of atomic elements are reduced in a system.

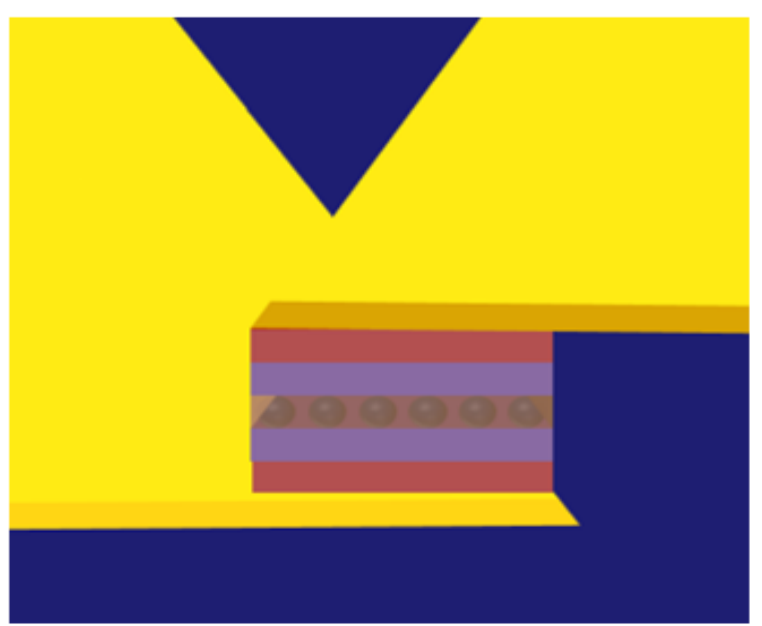

Figure 4-8: Particle Based MTJ Structure (left) and Schematic of Energy Levels with Fermi Energy Controlled by a Magnetic Field (right)

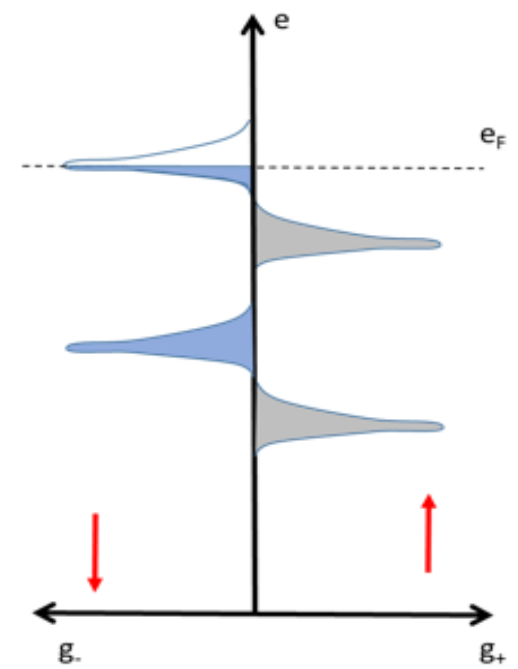

$g_{+}$ 


\section{Process Flow}

These devices follow the same fabrication steps as the regular film based MTJs. We inexpensively exploit the size of the nanoparticles without having to use expensive patterning methods to achieve nanometer scale devices. We do use FIB to trim the area down to ensure uniformity since these particles are prone to aggregation. Due to the clustering effect of the nanoparticles, we experienced a low yield in the fabrication process. However, there was data that supports the theory we had established regarding the size of the MTJ.
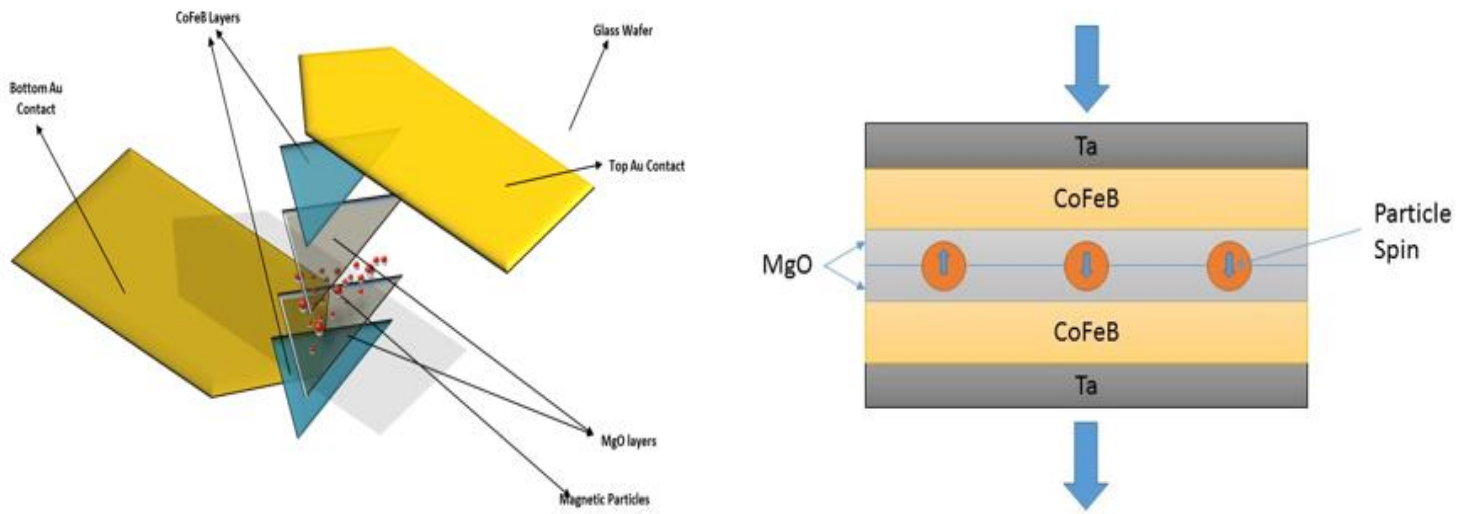

Figure 4-9: Thin Film Schematic of Particle Based Magnetic Tunnel Junction (left) and Cross-Sectional View of Particle Based Magnetic Tunnel Junction (right)

Ferrimagnetic $\mathrm{CoFe} 2 \mathrm{O} 4$ nanoparticles, synthesized through a chemical process (coprecipitation), were deposited onto the first $\mathrm{MgO}$ layer on the bottom contact. Then, the second $\mathrm{MgO}$ layer was deposited to separate the nanoparticles from the top contact. Magnetic force microscopy was done to verify the magnetic nature of the particle, and high-resolution transmission electron microscopy (TEM) was done to image the nanoparticle and check its crystallinity. 


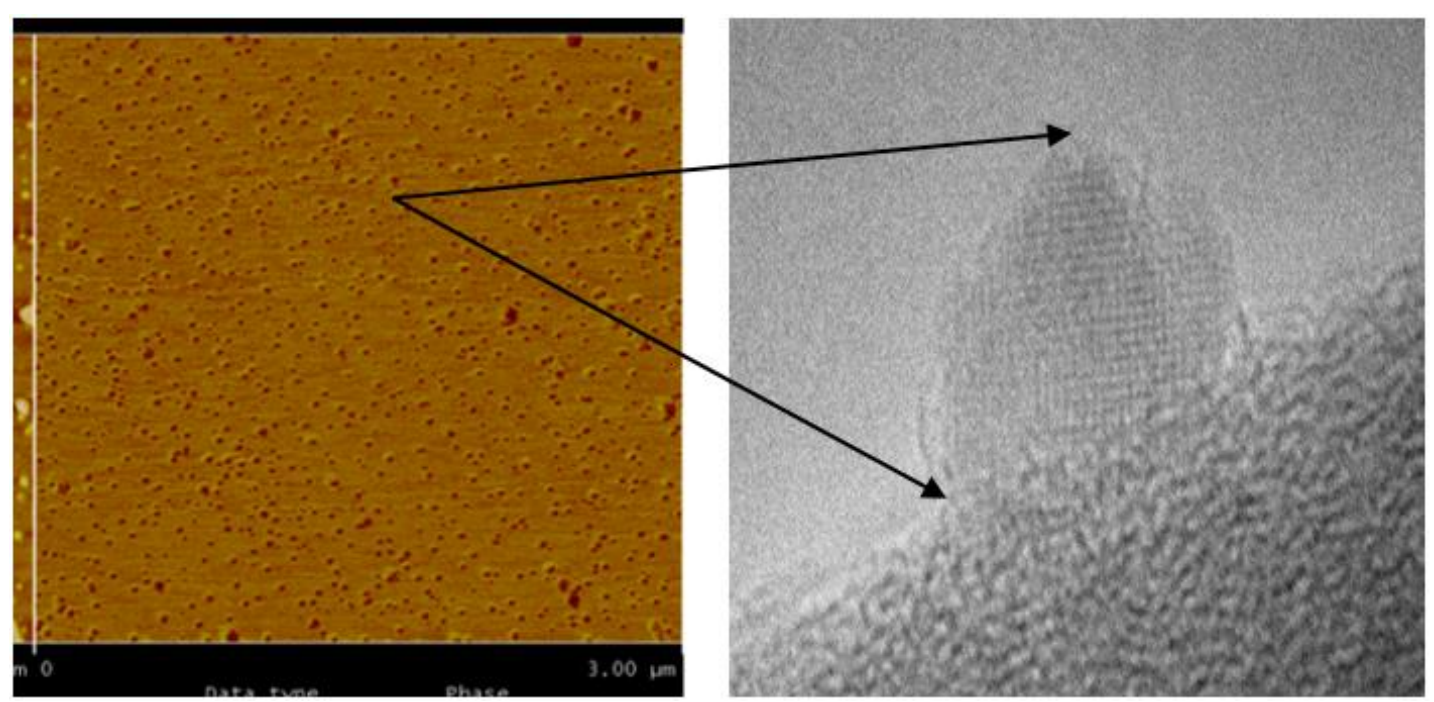

Figure 4-10: MFM Image (left) and High-resolution TEM Image of 2-nm CoFe2O4 Nanoparticles Sandwiched in a Junction (right)
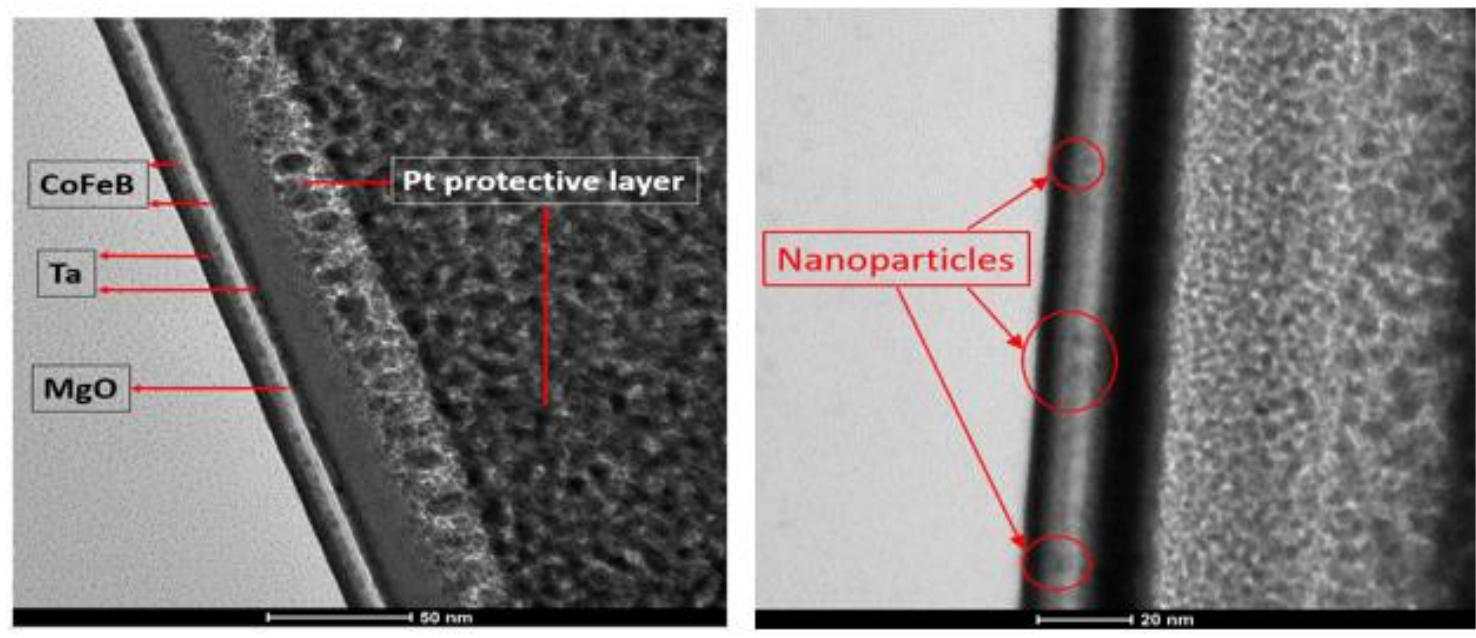

Figure 4-11: TEM Images of Nanoparticles MTJ (left) and Magnification to see nanoparticles (right)

Below are images of the particle based MTJ. The left image is a structure that has undergone FIB etching to improve the uniformity of the MTJ. The other two images are cross-sectional TEM images that were taken to verify the uniformity and roughness of the films sputtered. 

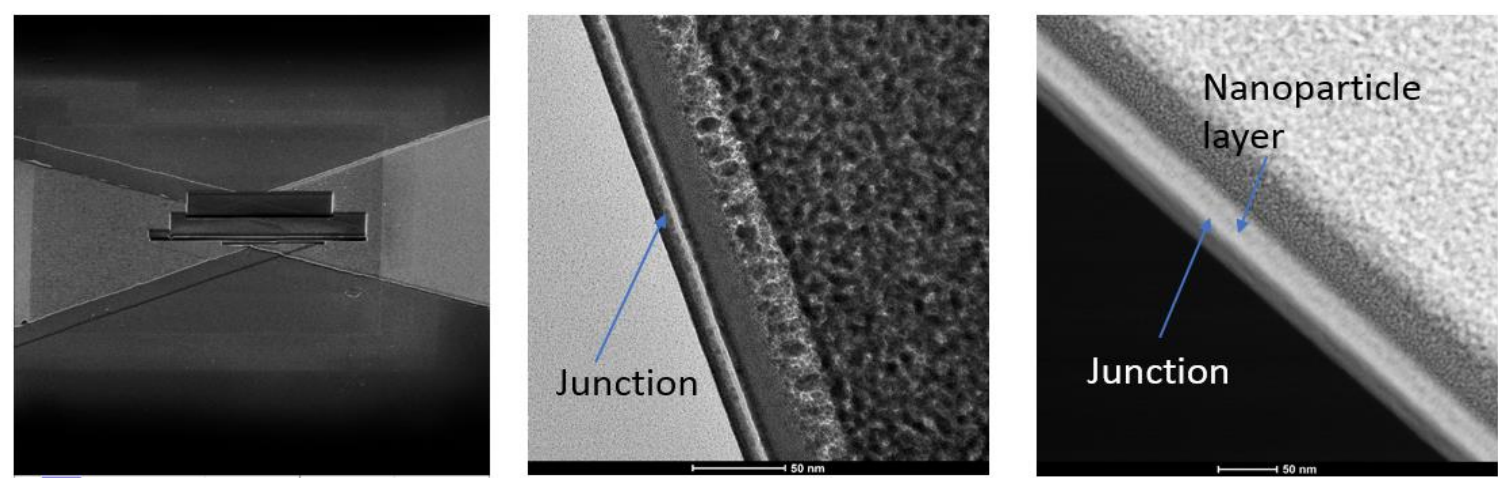

Figure 4-12: Gallium Fibbed Magnetic Tunnel Junction (left) and TEM Images of the Cross Section of the MTJ (middle \& right)

Each device fabricated has a different rate of aggregation and particles aren't necessarily uniformly spread out when deposited. Severe aggregations will yield no MR results (similar to 10nm). When we trim the devices, there is also a chance that we don't have a nanoparticle sandwiched between the junction. This will yield a highly resistive junction without any significant MR signal. The increased resistance is due to the way the particles and the films are deposited. The tunnel barrier is twice as thick across the electrodes than between the particle and the electrode. Tunneling resistance has an exponential relation to the thickness so therefore devices without particles are essentially open circuits.

\section{Results}

After fabricating and making many batches we found that non-zero MR was measured only with particles that were less than $10 \mathrm{~nm}$. The smaller the particles we used, the more likely the junction would exhibit an MR effect. Different concentrations of nanoparticles were also studied. High concentrations of particles are more susceptible to aggregation and tend to cluster more. This clustering effect both negates the sub $10 \mathrm{~nm}$ properties and creates hot spots in the junction, resulting in electrical shorts. We found that lower concentration of particles was the best route. 

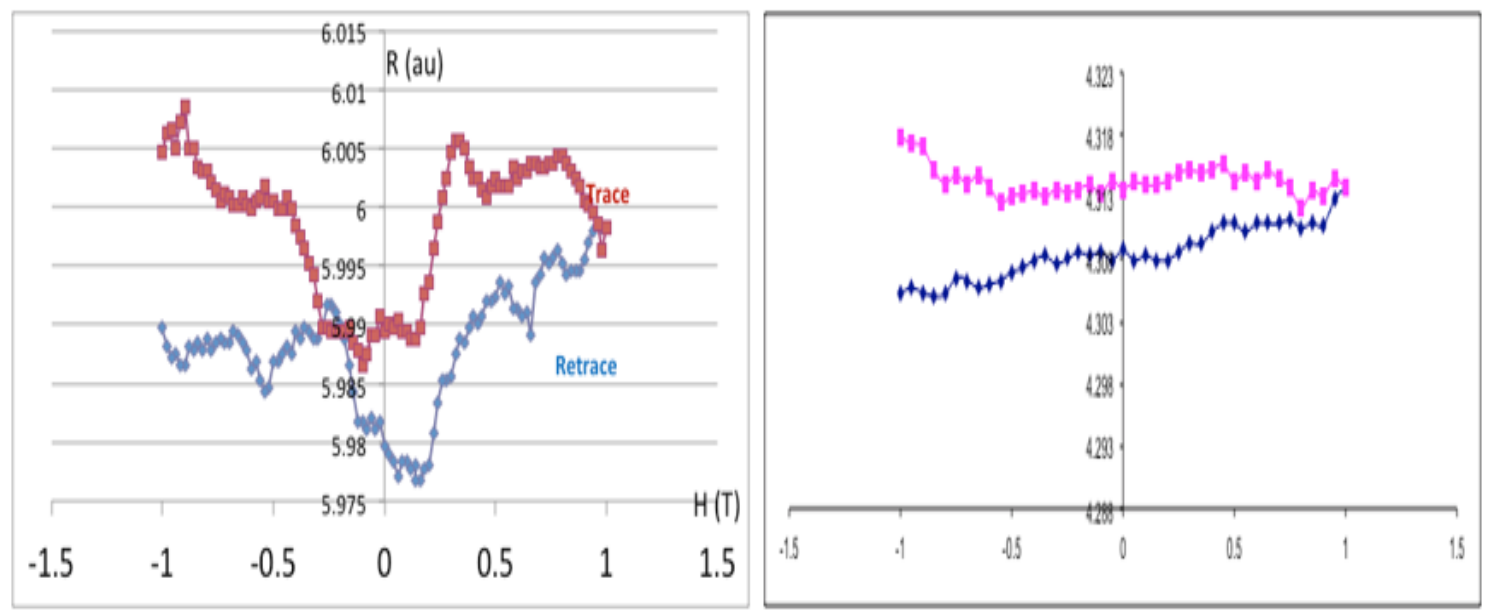

Figure 4-13: MR for 4nm particles (left) and 10nm particles (right)

Devices with $2 \mathrm{~nm}, 5 \mathrm{~nm}$, and $10 \mathrm{~nm}$ nanoparticles were made. The trend has been most reproducible with visible $\mathrm{MR}$ only with $2 \mathrm{~nm}$ and $4 \mathrm{~nm}$ nanoparticles. Ideally, a significantly larger “On/Off” ratio should be obtained. This concept offers a much more sensitive approach (a sensitivity in the order of a few Oersteds compared to hundreds of Oersteds with other approaches). The dissimilarity most likely is a combination of structural effects and the uniformity at which nanoparticles disperse in the tunnel junction.

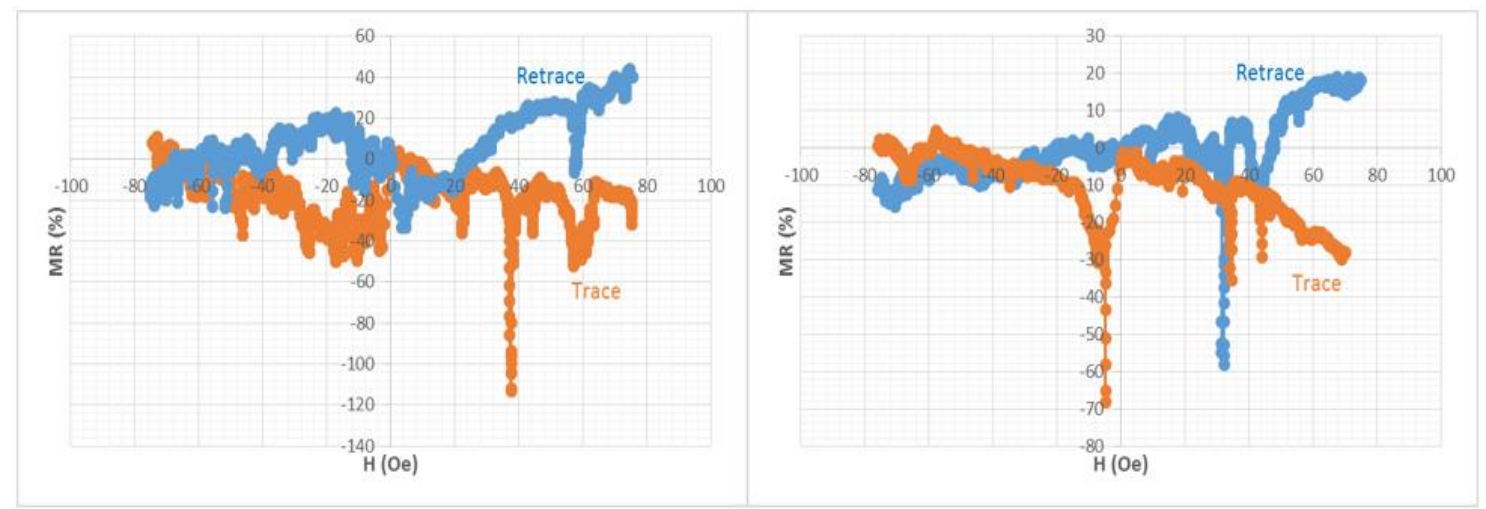

Figure 4-14: MR for 2nm Particles (Left and right graphs come from different batches)

All the transport measurements were conducted at room temperature. The curves were taken in the both current sweep directions, with the current increased from a negative value to a positive value and then decreased back to the negative value, defined as trace 
and retrace, respectively. The following IV curve (Figure 4-15) is from a matured process batch consisting of an MTJ with 2nm nanoparticles. The steps signaled with arrows are evidence of a standard Coulomb staircase, characteristic of single-electron transport in the tunnel junction.

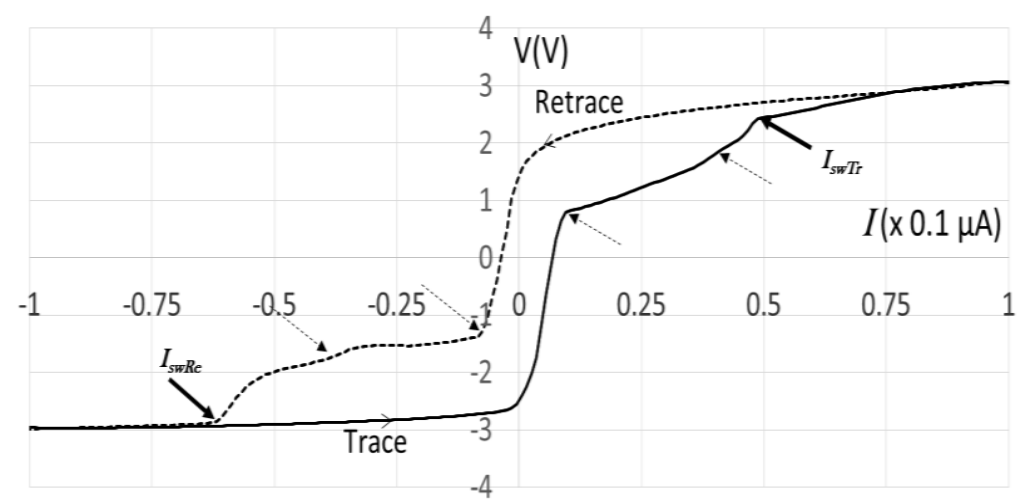

Figure 4-15: IV measurement of 2nm-Nanoparticle MTJ with evidence of single electron transport

However, this Coulomb staircase is only evident in half of each current sweep. This Coulomb blockade can be attributed to the presence of the two magnetic layers, where the relative orientation of the magnetization of the nanoparticle. This is illustrated in the following figure:
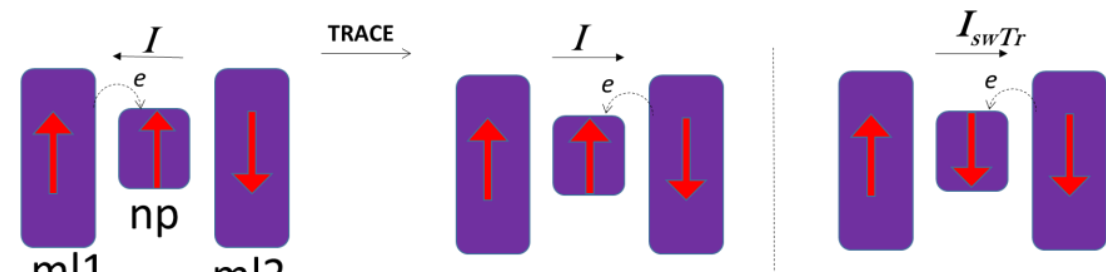

$\mathrm{ml} 1$

$\mathrm{ml} 2$
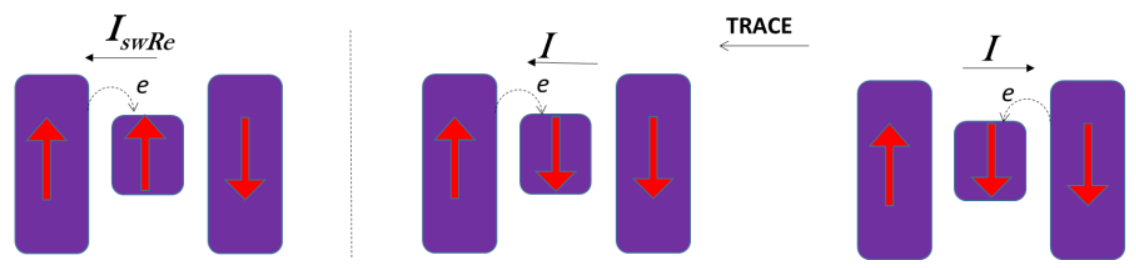

Figure 4-16: Nanoparticle magnetization with respect to the different points on the IV curve from Figure 415. 
To minimize the magnetic energy, the magnetization directions in the two CoFeB layers have anti-parallel but in-plane orientations, because of the dominant shape anisotropy. Although these directions don't ever change in this process, the magnetization of the nanoparticle can be oriented along any of the two anti-parallel orientations and can be switched between them through the STT effect. The interaction between each incoming electron and the electron currently sitting in the nanoparticle is affected by the relative orientation of the magnetization of the source magnetic layer and the nanoparticle magnetization. Due to the spin-dependent exchange coupling, the Coulomb repulsion for the parallel and antiparallel spin orientations will be effectively further increased and decreased, respectively. Therefore, in the parallel case, it will take a higher voltage to push the sitting electron away from the nanoparticle to the drain magnetic layer, compared to the antiparallel case.

Many IV measurements showed two windows of current with high resistance. Because we have a particle interfaced with two magnetic films, we are bound to have ternary logic. The following IV curve is another example of this occurrence. With the assumption that all the fields are initially magnetized in the same direction we find that the spin is more amenable to switching than the films, creating two antiparallel (AP) interfaces, resulting in the highest resistance. With enough current, the other films switch from a single AP interface to an all parallel interface. 


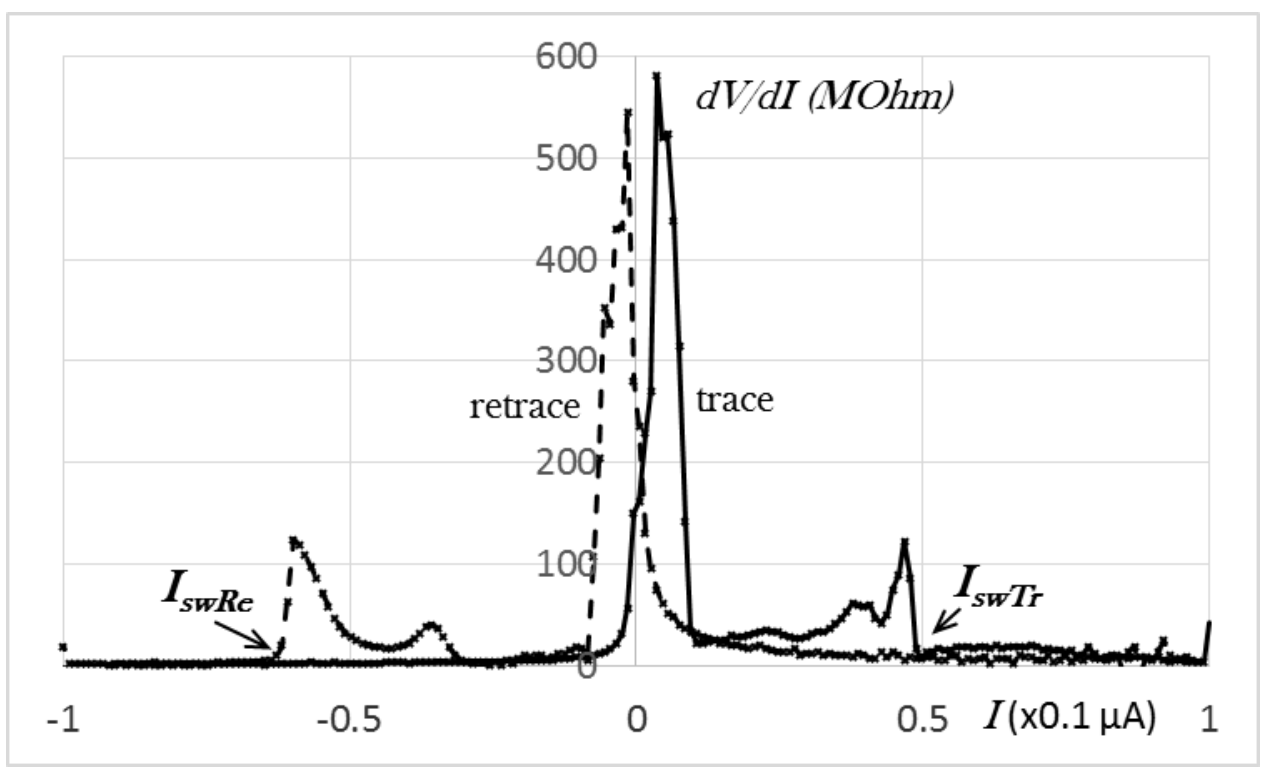

Figure 4-17: IV measurement for 2nm particles

When undergoing the MR measurement, there was a current dependence. For high currents $(50 \mathrm{nA})$, there was a reversible oscillatory dependence, particularly in the positive direction of the applied magnetic field. This effect isn't as noticeable for lower current values (10nA). It is possible that these values reflect the different regions in the Coulomb staircase in the IV curve. It is also possible that the applied field shifts the fermi level through the nanoparticles discrete energy levels, which would explain the sudden changes in resistance. 


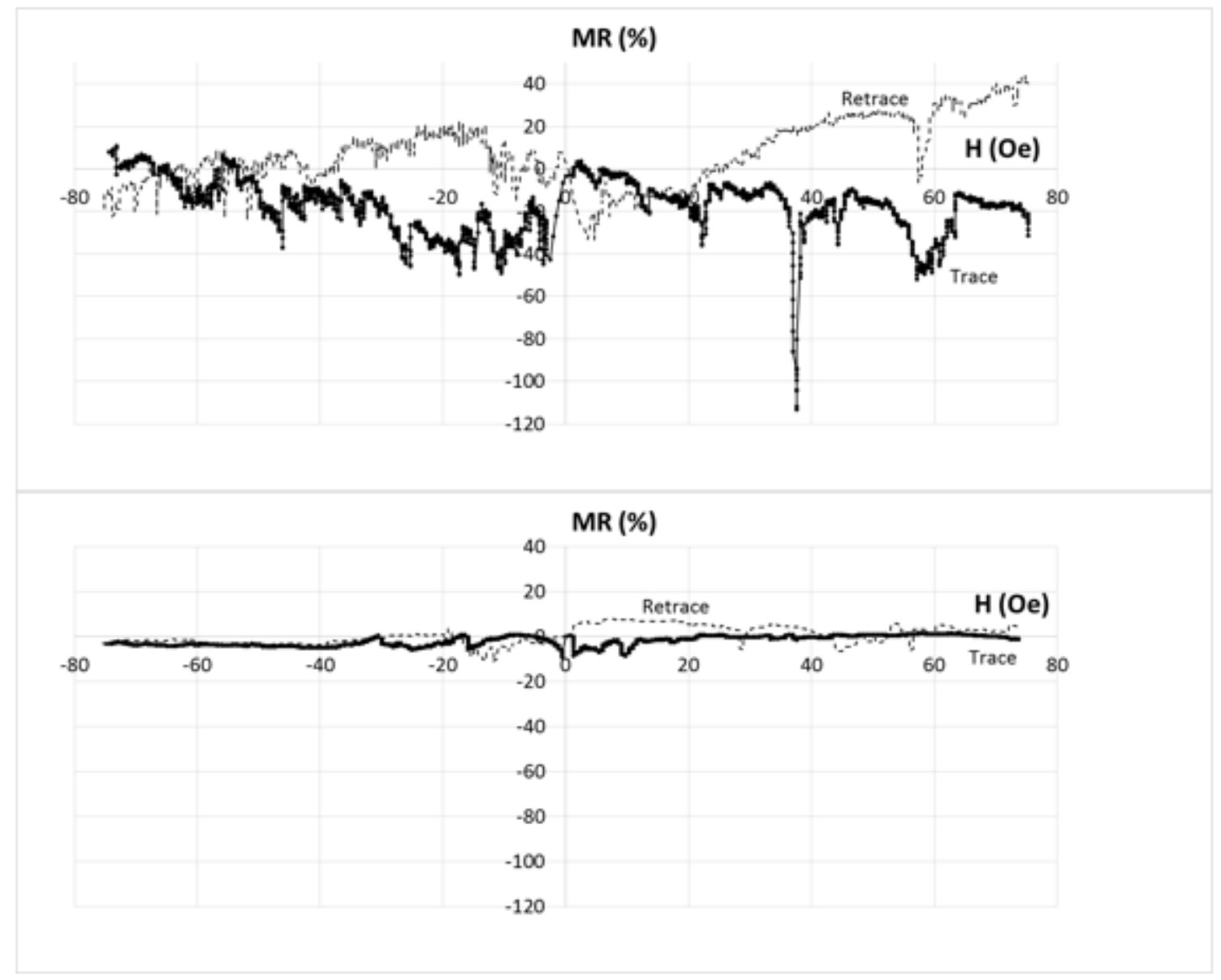

Figure 4-18: MR done at 50nA (top) and MR done at 10nA (bottom)

Because the high current value is above the first step on the Coulomb staircase, any application of the perpendicular magnetic field could shift steps through the value resulting in the resistance slumps. In contrast, because the small current value is already below or close to the first Coulomb step, we find that the external magnetic field barely affects the dependence. At high fields, no oscillations could be detected since the two magnetic films are parallel (due to saturation at a high magnetic field). This promotes the importance of the magnetic states of the two magnetic layers (effects are due the magnetic films being anti-parallel). 
These nanoparticle based MTJs exhibit room temperature, single electron transport. There is also a strong dependence on the applied magnetic field. The magnetic-field dependent characteristic makes this dual STT-MTJ design a suitable candidate for a twoterminal field-gated transistors for next-generation spintronic devices.

\subsection{Ternary 3D Logic}

Spintronic devices have the capability for three-dimensional information processing capability. Evidence supports the capability for these devices to be integrated in the third dimension as opposed to their semiconductor counterparts. FIB based MTJ devices were developed and stacked on each other, reducing the information processing footprint substantially. Sub 20nm devices were developed using a spin polarized current to switch magnetic states. This study was done to demonstrate the multiple level logic capability per cell that spin based devices have to offer.

\section{Theory}

Different size STT-MTJs were studied using a test structure that allowed using focused ion beam. We used (FIB) etching to define a planar geometry, as illustrated below. Together with Dr. Jeonming Hong, we fabricated a relatively large size junction with a characteristic planar size of over $1 \mu \mathrm{m}$ which. The standard perpendicular magnetic junction composition of $\mathrm{Ta} / \mathrm{CoFeB} / \mathrm{MgO}$ was used. The overall device composition was $\mathrm{Ta} / \mathrm{Ru} / \mathrm{Ta} / \mathrm{CoFeB}(\mathrm{M} 1) / \mathrm{MgO} / \mathrm{CoFeB}(\mathrm{M} 2) / \mathrm{MgO} / \mathrm{CoFeB}(\mathrm{M} 3) / \mathrm{Ta}$ [69]. FIB trimming was used to further reduce the planar sides below a 10-nm size, and rather than a gallium source, FIB was done with $\mathrm{He}$ and $\mathrm{Ne}$ ions. The advantages of $\mathrm{He} / \mathrm{Ne}$ ion based $\mathrm{FIB}$ is the ability to mill soft and fragile materials at low rates very precisely (effective probe 
sizes of $<0.5 \mathrm{~nm}$ for $\mathrm{He}$ and $<2 \mathrm{~nm}$ for $\mathrm{Ne}$ ) [70, 71]. Also using these alternative ions avoids the potential negative effects of Ga implantation in the material. Two junctions are placed in series to enable ternary information processing in a sub-10-nm nanomagnetic structure. The resistance depends on the relative orientation of the spin in the adjacent layers. The parallel orientation, $\mathrm{P}$, has a smaller resistance value compared to that for the anti-parallel orientation, AP. Consequently, two junctions provide three resistance values that correspond to the following combinations: (1) the low resistance value, R1, when both junctions are in P configuration, (2) the medium value, R2, when the two junctions are in $\mathrm{P}$ and $\mathrm{AP}$ configurations, respectively, and (3) the high value, R3, when both junctions are in AP configuration [69].

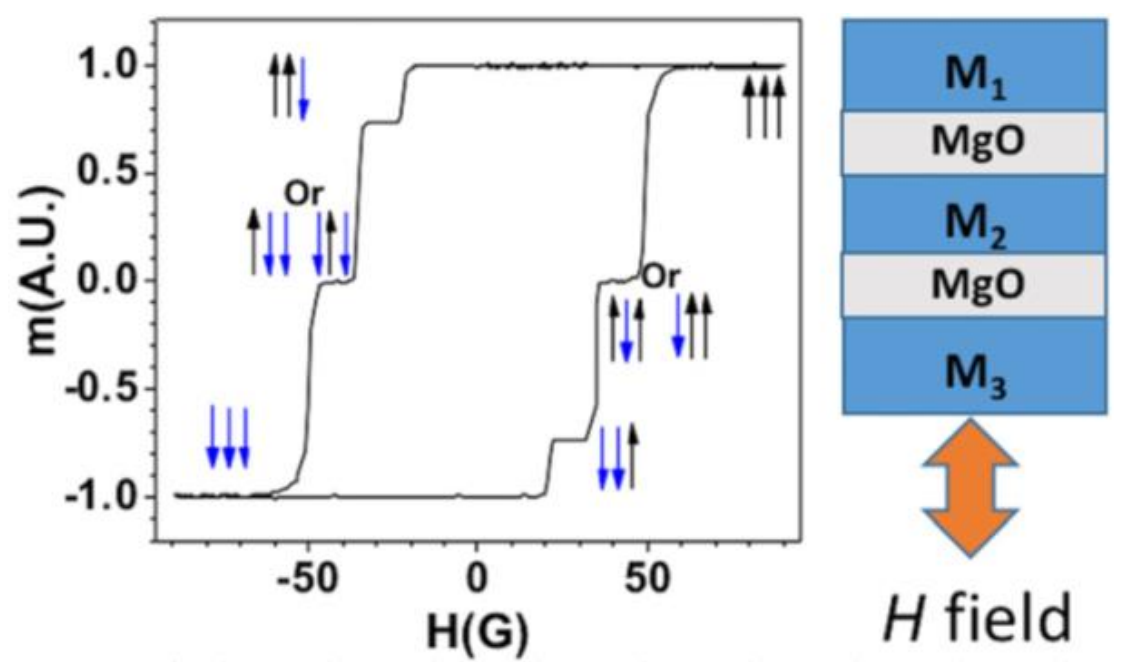

Figure 4-19: MH-Loop of MTJ Structure (left) and MTJ Composition (right) [69]

\section{Results}

A full I-V curve was measured by sweeping voltage from 0 to $+100 \mathrm{mV}$, from +100 to $100 \mathrm{mV}$, and from -100 to $0 \mathrm{mV}$. The measurement time at each point was $1 \mathrm{~ms}$. The first important observation is the fact the dependence can be described by three distinct linear curves with three resistance values, $R_{1}=46 \mathrm{KOhm}, \mathrm{R}_{2}=53 \mathrm{KOhm}, \mathrm{R}_{3}=82 \mathrm{KOhm}$, 
respectively. The system can be transitioned between these three states via application of certain voltage values. As the voltage is increased from zero $\left(\mathrm{V}_{0}\right)$ to the critical point $\left(\mathrm{V}_{\mathrm{c}}\right)$, no current could be driven. Then, all the way to point $\mathrm{A}(\mathrm{V} \sim 60 \mathrm{mV}, \mathrm{I} \sim 1000 \mathrm{nA})$, the dependence was strictly linear with the resistance value $\mathrm{R}_{2}$. As the voltage was further increased, the system moved to the curve with the lowest resistance value $\left(\mathrm{R}_{1}\right)$, i.e., from point A to point $\mathrm{B}(\mathrm{V} \sim 60 \mathrm{mV}, \mathrm{I} \sim 1200 \mathrm{nA})$. As the voltage was increased to the maximum point of $100 \mathrm{mV}(\mathrm{C})$, the system remained in the resistance state $\mathrm{R} 1$. The sequence can be clearly traced in the voltage reversed mode, i.e. from point $\mathrm{C}$ to point $\mathrm{L}$, and then back to the origin $\mathrm{V}_{\text {fin }}$. One can note a few intermediate transitions to the state with the highest resistance $\left(\mathrm{R}_{3}\right)$. From this state, the system always promptly goes back to the median resistance state (R2). The reversed sequence, i.e. as the voltage is first swept from 0 to $-100 \mathrm{mV}$, from -100 to $+100 \mathrm{mV}$, and then back from +100 to $0 \mathrm{mV}$, is also shown.
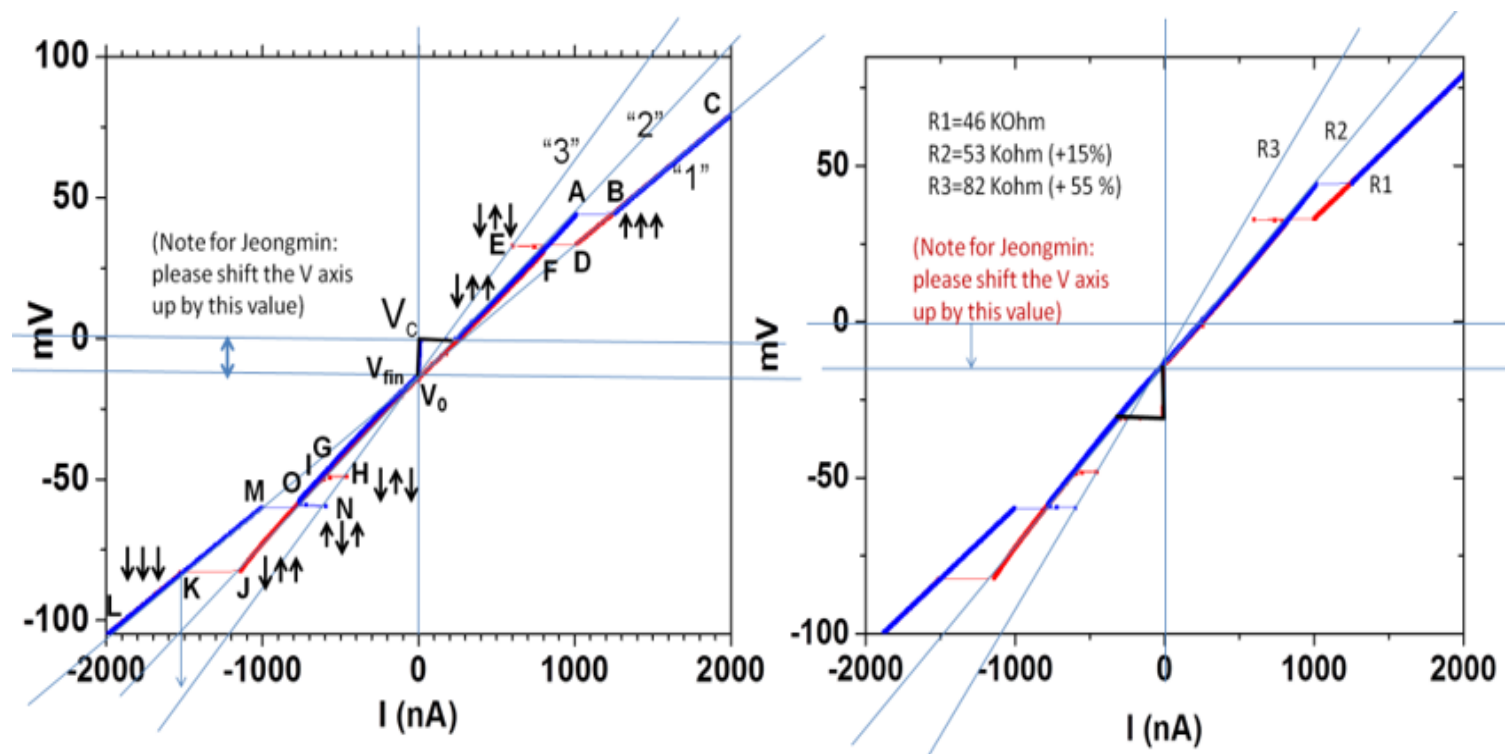

Figure 4-20: I-V curve measured via sweeping voltage in one direction (left) and the reverse direction (right) [69] 
The 3D device clearly yields a multilevel operation with a switching current density comparable to values published elsewhere in the sub-20nm range $\left(\sim 3 \mathrm{MA} / \mathrm{cm}^{2}\right)[48,60]$. In addition to spintronics being an all-purpose memory, it is possible to integrate these MTJ devices with current CMOS technology. These hybrid possibilities have real life industrial applications and can pave the path for future generations of spintronic devices. 


\section{CONCLUSION}

Exploring magnetic tunnel junctions in the sub-10nm range is an immense fabrication challenge. Optical lithography is limited to micrometer features and high resolution options (EBL, FIB, etc.) have low throughput and are expensive. Furthermore, many researchers have pushed the limit of electron beam lithography, and the smallest device recorded to date is still above 10nm. Conventional top-down approaches haven't made much progress in improving the size resolution of devices. This thesis focuses on coming up with innovative ways to circumvent the limitations of today's patterning technology. Clever techniques such as the nanoprobe MTJ is an example of novel methods of fabrication. The purpose of this research is not to make an industrial grade process with high yield, but to investigate the few devices that do come out and study the underlying physics. Eventually we will be able to scale devices in the sub-10nm range at an industrial level, so in the mean time we should put our efforts into understanding that size regime.

The focused ion beam (FIB) has allowed us to make devices around $5-7 \mathrm{~nm}$. We are limited in size of cuts we can make by the source of ions. Most of our devices were etched with a gallium ion source, however we did make use of a $\mathrm{Ne}$ and $\mathrm{He}$ source FIB in the latest generations. New sources with smaller elements, such as He, are emerging and will allow users to etch samples with angstrom resolution [71]. These finer beam sources will expand our ability to create even smaller devices! Another major focus of this thesis was exploiting the size of nanoparticles and embedding them into the MTJ architecture. There has been promising results in terms of improved on/ratios (theoretically $\infty$ ) that will make it a candidate as a near-perfect substitute transistor. There are many aspects of 
these devices that we must account for: the concentration, the medium that disperses the particles on the junction, cleanliness, aggregation, etc. Further research is necessary to properly characterize and optimize the process involved in embedding the particles. Another approach for making MTJs that has captivated our research group is the possibility of using nano-porous alumina. Nano-porous alumina has a highly ordered set of pores that can range from nanometers to micrometers. Taking advantage of a material that already has a pre-determined size is another avenue worth exploring. This can be done by using the porous alumina as a mask, electroplate contacts through the pores, chemical polishing, etc. It is our duty as researchers to explore beyond the boundary constraints. While top-down methods are well established, there are opportunities to exploit bottom-up approaches with novel materials.

The purpose of this research is to explore the poorly understood size range $(<10 \mathrm{~nm})$ of magnetics where we theorize a significant improvement in device performance. We have discovered that the switching current density should be reduced, the magnetoresistance increased and thus overall energy efficiency increased as the device size is reduced below $\sim 10 \mathrm{~nm}$ (Figure 5-1). The surprising switching current reduction and MR increase are explained by the new physics described in chapter 4 . With such a small thermal reservoir, the model of continuous crystalline structure becomes invalid, which in turn leads to slow relaxation time and magnetization damping decrease. We should continue building sub10nm MTJs and studying the quantum-mechanical effects that are dominant in this size regime. 


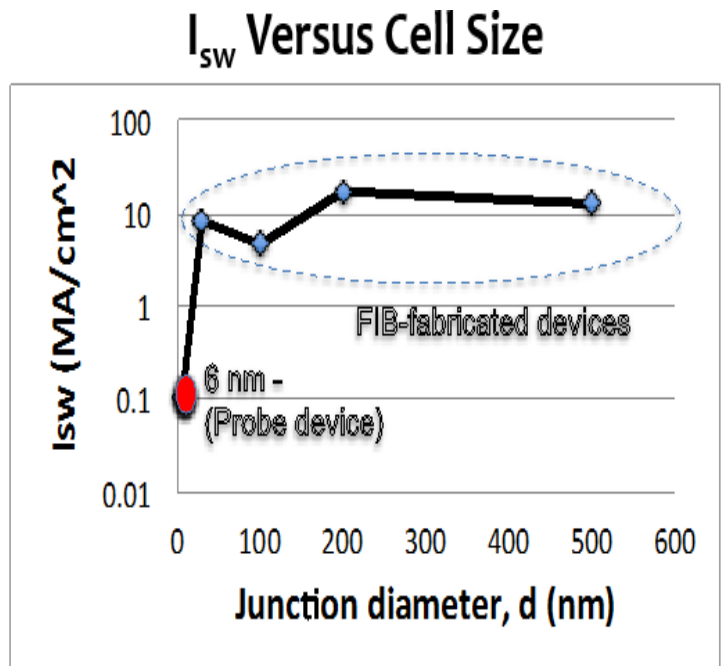

TMR Vs Cell size

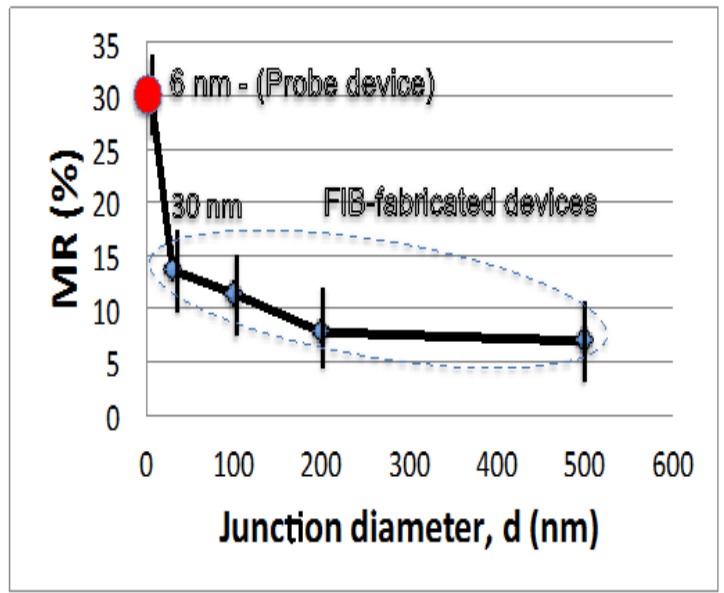

Figure 5-1: Switching Currents and TMR from Fabricated Devices 


\section{REFERENCES}

[1] J. M. Shalf and R. Leland, "Computing beyond Moore's Law," Mc, vol. 48, (12), pp. 14-23, 2015.

[2] Jongyeon Kim et al, "Spin-Based Computing: Device Concepts, Current Status, and a Case Study on a High-Performance Microprocessor," Proc. IEEE, vol. 103, (1), pp. 106$130,2015$.

[3] M. Tatchell-Evans et al, "An experimental and theoretical investigation of the extent of bypass air within data centres employing aisle containment, and its impact on power consumption," Applied Energy, vol. 186, pp. 457-469, 2017.

[4] R Landauer, "Irreversibility and heat generation in the computing process," IBM Journal of Research and Development, vol. 44, (1/2), pp. 261, 2000.

[5] L. Chang, M. Wang, L. Liu, S. Luo, P. Xiao, "A brief introduction to giant magnetoresistance," Beijing, China: Hycorelle Co. Ltd. 2014.

[6] Aakash Pushp et al, "Giant thermal spin-torque-assisted magnetic tunnel junction switching," Proceedings of the National Academy of Sciences, vol. 112, (21), pp. 6585, 2015.

[7] D. A. Thompson and J. S. Best, "The future of magnetic data storage technology," IBM Journal of Research and Development, vol. 44, (3), pp. 311, 2000.

[8] M. Gajek et al, "Spin torque switching of $20 \mathrm{~nm}$ magnetic tunnel junctions with perpendicular anisotropy," Applied Physics Letters, vol. 100, (13), pp. 132408, 2012.

[9] J. Hong et al, "Energy-efficient spin-transfer torque magnetization reversal in sub-10nm magnetic tunneling junction point contacts," J Nanopart Res, vol. 15, (4), pp. 1-6, 2013.

[10] V. L. Safonov, Nonequilibrium Magnons. Berlin, Germany: Wiley, 2012.

[11] M. Tsoi et al, "Excitation of a Magnetic Multilayer by an Electric Current," Physical Review Letters, vol. 80, (19), pp. 4281-4284, 1998.

[12] J. C. Slonczewski, "Currents and torques in metallic magnetic multilayers," Journal of Magnetism and Magnetic Materials, vol. 247, (3), pp. 324-338, 2002.

[13] L. Berger, "Emission of spin waves by a magnetic multilayer traversed by a current," Physical Review B, vol. 54, (13), pp. 9353-9358, 1996.

[14] N. A. Spaldin, Magnetic Materials. (2nd ed.) 2011.

[15] B. D. Cullity and C. D. Graham, Introduction to Magnetic Materials. (2nd ed.) Piscataway, NJ: IEEE Press [u.a.], 2009.

[16] J. M. D. Coey, Magnetism and Magnetic Materials. (1st ed.) GB: Cambridge University Press - M.U.A, 2009. 
[17] C. P. Landee and M. M. Turnbull, "Review: A gentle introduction to magnetism: units, fields, theory, and experiment," Journal of Coordination Chemistry, vol. 67, (3), pp. 375-439, 2014.

[18] A. L. Bhuiyan, "Effect of the strong field on the magnitude of the Bohr magneton," Physics Essays, vol. 18, (3), pp. 309, 2005.

[19] W. Kutzelnigg and J. D. Morgan, "Hund's rules," Zeitschrift F R Physik D Atoms, Molecules and Clusters, vol. 36, (3-4), pp. 197-214, 1996.

[20] Paul Forman, "Alfred Landé and the Anomalous Zeeman Effect, 19191921," Historical Studies

[21] M. A. Ruderman and C. Kittel, "Indirect Exchange Coupling of Nuclear Magnetic Moments by Conduction Electrons," Physical Review, vol. 96, (1), pp. 99-102, 1954.

[22] G. C. Papaefthymiou, "Nanoparticle magnetism," Nano Today, vol. 4, (5), pp. 438447, 2009.

[23] W. CHIU et al, "Tunable coercivity of CoFe2O4 nanoparticles via thermal annealing treatment," Journal of Alloys and Compounds, vol. 459, (1-2), pp. 291-297, 2008.

[24] P. J. Kelly and R. D. Arnell, "Magnetron sputtering: a review of recent developments and applications," Vacuum, vol. 56, (3), pp. 159-172, 2000.

[25] Baibich, M., Broto, J., Fert, A., Van Dau, F., Petroff, F., Etienne, P., Creuzet, G., Friederich, A. and Chazelas, J. (1988). Giant Magnetoresistance of (001)Fe/(001)Cr Magnetic Superlattices. Physical Review Letters, 61(21), pp.2472-2475.

[26] McGuire, T. and Potter, R. (1975). Anisotropic magnetoresistance in ferromagnetic 3d alloys. IEEE Transactions on Magnetics, 11(4), pp.1018-1038.

[27] Valet, T. and Fert, A. (1993). Theory of the perpendicular magnetoresistance in magnetic multilayers. Physical Review B, 48(10), pp.7099-7113.

[28] Fert, A. (2008). The present and the future of spintronics. Thin Solid Films, 517(1), pp. $2-5$.

[29] M. Julliere, "Tunneling between ferromagnetic films," Physics Letters A, vol. 54, (3), pp. 225-226, 1975.

[30] Wang, D., Nordman, C., Daughton, J., Qian, Z., Fink, J., Wang, D., Nordman, C., Daughton, J., Qian, Z. and Fink, J. (2004). 70\% TMR at Room Temperature for SDT Sandwich Junctions With $\mathrm{CoFeB}$ as Free and Reference Layers. IEEE Transactions on Magnetics, 40(4), pp.2269-2271. 
[31] Butler, W., Zhang, X., Schulthess, T. and MacLaren, J. (2001). Spin-dependent tunneling conductance ofFe $|\mathrm{MgO}|$ Fesandwiches. Physical Review B, 63(5).

[32] Mathon, J. and Umerski, A. (2001). Theory of tunneling magnetoresistance of an epitaxial Fe/MgO/Fe(001) junction. Physical Review B, 63(22).

[33] Y. Qi, D. Y. Xing and J. Dong, "Relation between Julliere and Slonczewski models of tunneling magnetoresistance," Physical Review B, vol. 58, (5), pp. 2783-2787, 1998.

[34] S. Ikeda, J. Hayakawa, Y. Ashizawa, Y. M. Lee, K. Miura, H. Hasegawa, M. Tsunoda, F. Matsukura, H. Ohno "Tunnel magnetoresistance of $604 \%$ at $300 \mathrm{~K}$ by suppression of $\mathrm{Ta}$ diffusion in $\mathrm{CoFeB} / \mathrm{MgO} / \mathrm{CoFeBCoFeB} / \mathrm{MgO} / \mathrm{CoFeB}$ pseudo-spinvalves annealed at high temperature," Applied Physics Letters, 93, 082508, 2008.

[35] Slonczewski, J. (1996). Current-driven excitation of magnetic multilayers. Journal of Magnetism and Magnetic Materials, 159(1-2), pp.L1-L7.

[36] Katine, J., Albert, F., Buhrman, R., Myers, E. and Ralph, D. (2000). Current-Driven Magnetization Reversal and Spin-Wave Excitations in $\mathrm{Co} / \mathrm{Cu} / \mathrm{Co}$ Pillars. Physical Review Letters, 84(14), pp.3149-3152.

[37] Myers, E. (1999). Current-Induced Switching of Domains in Magnetic Multilayer Devices. Science, 285(5429), pp.867-870.

[38] Tsoi, M., Jansen, A., Bass, J., Chiang, W., Tsoi, V., Wyder, P. “Generation and detection of phase-coherent current-driven magnons in magnetic multilayers," Nature, 406(6791), pp.46-48, 2000.

[39] Christof Melcher and Mariya Ptashnyk, "Landau--Lifshitz--Slonczewski Equations: Global Weak and Classical Solutions," SIAM Journal on Mathematical Analysis, vol. 45, (1), pp. 407-429, 2013.

[40] Hayakawa, J., Ikeda, S., Lee, Y., Sasaki, R., Meguro, T., Matsukura, F., Takahashi, H. and Ohno, H., "Current-Driven Magnetization Switching in $\mathrm{CoFeB} / \mathrm{MgO} / \mathrm{CoFeB}$ Magnetic Tunnel Junctions," Japanese Journal of Applied Physics, 44(No. 41), pp.L1267-L1270, 2005.

[41] Kubota, H., Fukushima, A., Ootani, Y., Yuasa, S., Ando, K., Maehara, H., Tsunekawa, K., Djayaprawira, D., Watanabe, N. and Suzuki, Y., "Evaluation of SpinTransfer Switching in $\mathrm{CoFeB} / \mathrm{MgO} / \mathrm{CoFeB}$ Magnetic Tunnel Junctions," Japanese Journal of Applied Physics, 44(No. 40), pp.L1237-L1240, 2005.

[42] M. J. Madou, Fundamentals of Microfabrication. (2nd ed.) 2002.

[43] Lieberman, M. and Lichtenberg, A. (1994). Principles of plasma discharges and materials processing. New York: Wiley. 
[44] K. Seshan, Handbook of Thin Film Deposition. (3rd ed.) 2012.

[45] O'Hanlon, J. (2005). A User's Guide to Vacuum Technology. Hoboken: John Wiley $\&$ Sons, Inc.

[46] S. Tegen et al, "Effect of Néel coupling on magnetic tunnel junctions," Journal of Applied Physics, vol. 89, (12), pp. 8169-8174, 2001. . DOI: 10.1063/1.1365445.

[47] Namita Rajput, "METHODS OF PREPARATION OF NANOPARTICLES - A REVIEW," International Journal of Advances in Engineering \& Technology, vol. 7, (6), pp. 1806, 2015.

[48] Hong, J., Hadjikhani, A., Stone, M., Allen, F., Safonov, V., Liang, P., Bokor, J. and Khizroev, S. (2016). The Physics of Spin-Transfer Torque Switching in Magnetic Tunneling Junctions in Sub-10 nm Size Range. IEEE Transactions on Magnetics, 52(7), pp.1-4.

[49] Moodera, J., Kinder, L., Wong, T. and Meservey, R. (1995). Large Magnetoresistance at Room Temperature in Ferromagnetic Thin Film Tunnel Junctions. Physical Review Letters, 74(16), pp.3273-3276.

[50] Miyazaki, T. and Tezuka, N. (1995). Giant magnetic tunneling effect in Fe/Al2O3/Fe junction. Journal of Magnetism and Magnetic Materials, 139(3), pp.L231L234.

[51] J. Zhu and C. Park, "Magnetic tunnel junctions," Materials Today, vol. 9, (11), pp. 36-45, 2006.

[52] S. Yuasa et al, "High Tunnel Magnetoresistance at Room Temperature in Fully Epitaxial $\mathrm{Fe} / \mathrm{MgO} / \mathrm{Fe}$ Tunnel Junctions due to Coherent Spin-Polarized Tunneling," Japanese Journal of Applied Physics, vol. 43, (No. 4B), pp. L590, 2004.

[53] C. Kaiser et al, "Giant tunneling magnetoresistance at room temperature with $\mathrm{MgO}$ (100) tunnel barriers," Nature Materials, vol. 3, (12), pp. 862-867, 2004.

[54] Zhang, X. and Butler, W. (2004). Large magnetoresistance in bccCoMgO/CoandFeCo/MgO/FeCo tunnel junctions. Physical Review B, 70(17).

[55] S. Yuasa et al, "230\% room-temperature magnetoresistance in $\mathrm{CoFeB} / \mathrm{MgO} /$ CoFeB magnetic tunnel junctions," Applied Physics Letters, vol. 86, (9), pp. 3, 2005.

[56] Yuasa, S., Suzuki, Y., Katayama, T. and Ando, K. (2005). Characterization of growth and crystallization processes in $\mathrm{CoFeB} / \mathrm{MgO} / \mathrm{CoFeB}$ magnetic tunnel junction structure by reflective high-energy electron diffraction. Applied Physics Letters, 87(24), p.242503.

[57] Yuasa, S. and Djayaprawira, D. (2007). Giant tunnel magnetoresistance in magnetic tunnel junctions with a crystalline $\mathrm{MgO}(001)$ barrier. Journal of Physics D: Applied Physics, 40(21), pp.R337-R354. 
[58] Choi, Y., Tsunekawa, K., Nagamine, Y. and Djayaprawira, D. (2007). Transmission electron microscopy study on the polycrystalline $\mathrm{CoFeB} M g \mathrm{O} / \mathrm{CoFeB}$ based magnetic tunnel junction showing a high tunneling magnetoresistance, predicted in single crystal magnetic tunnel junction. Journal of Applied Physics, 101(1), p.013907.

[59] Chen, E., Apalkov, D., Driskill-Smith, A., Khvalkovskiy, A., Lottis, D., Moon, K., Nikitin, V., Ong, A., Tang, X., Watts, S., Kawakami, R., Krounbi, M., Wolf, S., Poon, S., Lu, J., Ghosh, A., Stan, M., Butler, W., Mewes, T., Gupta, S., Mewes, C., Visscher, P. and Lukaszew, R., "Progress and Prospects of Spin Transfer Torque Random Access Memory," IEEE Transactions on Magnetics, 48(11), pp.3025-3030, 2012.

[60] Sato, H., Ikeda, S. and Ohno, H. (2017). Magnetic tunnel junctions with perpendicular easy axis at junction diameter of less than $20 \mathrm{~nm}$. Japanese Journal of Applied Physics, 56(8), pp.0802A6.

[61] Sbiaa, R., Meng, H. and Piramanayagam, S. "Materials with perpendicular magnetic anisotropy for magnetic random access memory," physica status solidi (RRL) - Rapid Research Letters, 5(12), pp.413-419, 2011.

[62] S. H. Charap, "Thermal stability of recorded information at high densities," IEEE Trans. Magn., vol. 33, no. 1, p. 978, 1997.

[63] Hamann, H., Martin, Y. and Wickramasinghe, H. (2004). Thermally assisted recording beyond traditional limits. Applied Physics Letters, 84(5), pp.810-812.

[64] J. Nowak et al, "Size dependence of spin-torque induced magnetic switching in CoFeB-based perpendicular magnetization tunnel junctions (invited)," Journal of Applied Physics, vol. 111, (7), pp. 3, 2012.

[65] Ikeda, S. (2010). "A perpendicular anisotropy $\mathrm{CoFeB}-\mathrm{MgO}$ magnetic tunnel junction." Nat. Mater, 9, pp.721-724.

[66] J. Hong, P. Liang, V. L. Safonov, and S. Khizroev, "Energy-efficient spin transfer torque magnetization reversal in sub-10-nm magnetic tunneling junction point contacts," J. Nanoparticle Res., vol. 15, no. 4, pp. 1-6, 2013.

[67] J. C. Slonczewski and J. Z. Sun, "Theory of voltage-driven current and torque in magnetic tunnel junctions," Journal of Magnetism and Magnetic Materials, vol. 310, (2), pp. 169-175, 2007.

[68] N. M. Caffrey et al, "Spin-filtering efficiency of ferrimagnetic spinels $\mathrm{CoFe} 2 \mathrm{O} 4$ and NiFe2O4," 2013.

[69] Hong, J., Stone, M., Navarrete, B., Luongo, K., Zheng, Q., Yuan, Z., Xia, K., Xu, N., Bokor, J., You, L. and Khizroev, S. (2018). 3D multilevel spin transfer torque devices. Applied Physics Letters, 112(11), p.112402.

[70] Tan, S., Livengood, R., Hack, P., Hallstein, R., Shima, D., Notte, J. and McVey, S. (2011). Nanomachining with a focused neon beam: A preliminary investigation for 
semiconductor circuit editing and failure analysis. Journal of Vacuum Science \& Technology B, Nanotechnology and Microelectronics: Materials, Processing, Measurement, and Phenomena, 29(6), pp.06F604.

[71] G. Hlawacek, V. Veligura, R. van Gastel, and B. Poelsema, "Helium ion microscopy," J. Vac. Sci. Technol. B, vol. 32, no. 2, p. 020801, 2014. 
VITA

\section{MARK STONE}

\section{EDUCATION}

Ph.D. Candidate in Electrical Engineering (2013-2018)

Florida International University (GPA: 3.93/4.0)

Bachelor of Science in Electrical Engineering (2008-2013)

Florida International University (GPA: 3.69/4.0)

Honors College Graduate

\section{PUBLICATIONS AND PRESENTATIONS}

- J. Hong, A. Hadjikhani, M. Stone, F. Allen, V. Safonov, P. Liang, J. Bokor, and S. Khizroev, "The physics of spin-transfer torque switching in magnetic tunneling junctions in sub-10-nm size range," IEEE Trans. Magn. 52 (7), 1400504 (2016)

- M. Stone et al., "Anomalous properties of sub-10-nm magnetic tunneling junctions," 2015 Fourth Berkeley Symposium on Energy Efficient Electronic Systems (E3S), Berkeley, CA, 2015, pp. 1-3

- M. Stone, B. Navarette, K. Luongo, A. Hadjikhani, P. Wang, P. Liang, S. Khizroev, "Anomalous magnetoresistance oscillations in magnetic tunneling junctions with embedded magnetic metal nanoparticles," Appl. Phys. Lett., in press, 2017. (Submitted)

- J. Hong, M. Stone, B. Navarette, K. Luongo, Q. Zheng, Z. Yuan, K. Xia, N. Xu, J. Bokor, S. Khizroev, "3D Multilevel spin transfer torque devices,” Applied Physics Letters, 2018. 